\title{
Search for a Standard Model Higgs Boson with a Dilepton and Missing Energy Signature
}

\author{
Davide Gerbaudo
}

A DISSERTATION PRESENTED TO THE

FACULTY OF PRINCETON UNIVERSITY

IN CANDIDACY FOR THE DEGREE OF DOCTOR OF PHILOSOPHY

RECOMMENDED FOR ACCEPTANCE

BY THE DEPARTMENT OF PHYSICS

Advisor: Christopher G. Tully

September 2011 
(c) Copyright by Davide Gerbaudo, 2011. All rights reserved. 


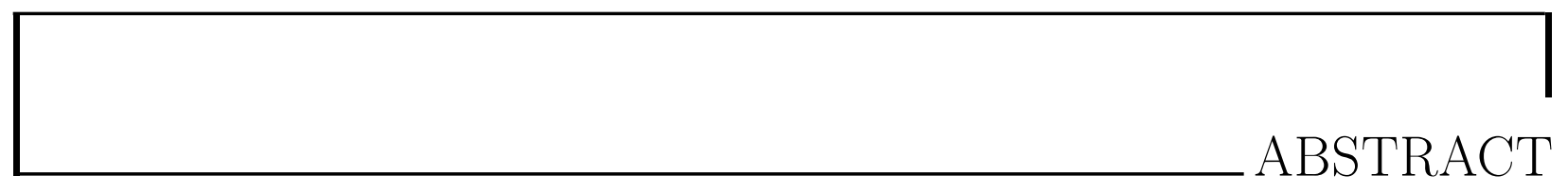

The subject of this thesis is the search for a standard model Higgs boson decaying to a pair of $\mathrm{W}$ bosons that in turn decay leptonically, $\mathrm{H} \rightarrow \mathrm{W}^{+} \mathrm{W}^{-} \rightarrow \bar{\ell} \nu \ell \bar{\nu}$. This search is performed considering events produced in $\mathrm{p} \overline{\mathrm{p}}$ collisions at $\sqrt{s}=1.96 \mathrm{TeV}$, where two oppositely charged lepton candidates $\left(\mathrm{e}^{+} \mathrm{e}^{-}, \mathrm{e}^{ \pm} \mu^{\mp}\right.$, or $\left.\mu^{+} \mu^{-}\right)$, and missing transverse energy, have been reconstructed. The data were collected with the $\mathrm{D} \varnothing$ detector at the Fermilab Tevatron collider, and are tested against the standard model predictions computed for a Higgs boson with mass in the range $115-200 \mathrm{GeV}$. No excess of events over background is observed, and limits on Standard Model Higgs boson production are determined. An interpretation of these limits within the hypothesis of a fourth-generation extension to the standard model is also given. The overall analysis scheme is the same for the three dilepton pairs being considered $\left(\mathrm{e}^{+} \mathrm{e}^{-}, \mathrm{e}^{ \pm} \mu^{\mp}\right.$, or $\left.\mu^{+} \mu^{-}\right)$; this thesis, however, describes in detail the study of the dimuon final state. 
The work described in this dissertation

has been published in the following journals:

Phys.Rev.Lett. 104, 061804 (2010)

Phys.Rev.Lett. 104, 061802 (2010)

Phys. Rev. D 82, 011102 (2010)

arXiv:1103.3233 [hep-ex] 


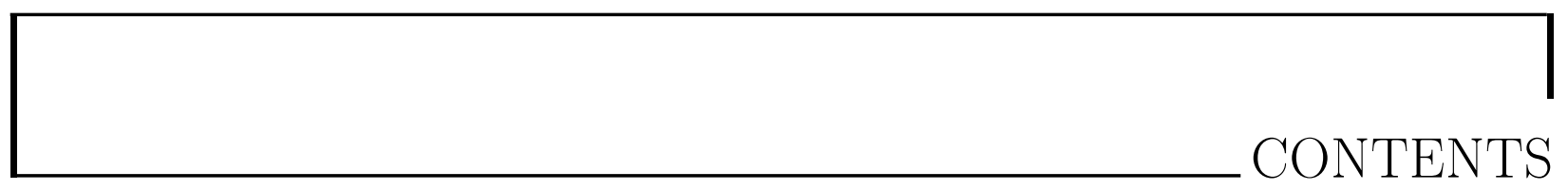

$\begin{array}{ll}\text { Abstract } & \text { iii }\end{array}$

$\begin{array}{ll}\text { Acknowledgments } & \text { ix }\end{array}$

Ringraziamenti $\quad$ x

List of Abbreviations xiii

1 Introduction $\quad 2$

1.1 Motivation . . . . . . . . . . . . . . . . . . . 2

1.1.1 Construction and Limitations of the Standard Model . . . . . . . . . 2

1.1.2 Why Search for the Higgs Boson at the Tevatron . . . . . . . . . . . 4

1.2 The Standard Model . . . . . . . . . . . . . . . . . . 5

1.2.1 Subatomic Matter and Forces ................ 5

1.2.2 Gauge Symmetries . . . . . . . . . . . . . . . 7

1.3 The Higgs Boson . . . . . . . . . . . . . . . . . . . . . . . . . . . . 8

1.3.1 Symmetry Breaking and the Higgs Boson . . . . . . . . . . . . . . 8

1.3.2 Properties of the Higgs Boson . . . . . . . . . . . . . . . . 9

1.3.3 Strategy for a Higgs Boson Search . . . . . . . . . . . . . . . . . . 14 
2 Hadron Collider Physics at the Tevatron $\quad 17$

2.1 Coordinates and Units . . . . . . . . . . . . . . . . . . 17

2.2 Proton-Antiproton Collisions at the Tevatron . . . . . . . . . . . . . 19

2.2.1 The Accelerator Chain . . . . . . . . . . . . . . . . . . 19

2.2.2 The Tevatron Main Ring . . . . . . . . . . . . . . . . 21

2.3 High- $p_{\mathrm{T}}$ Physics . . . . . . . . . . . . . . . . . . . 21

2.3.1 Typical Detector . . . . . . . . . . . . . . . . 22

2.3.2 Typical Problems at a Hadron Collider Experiment . . . . . . . . . . 24

3 Event Reconstruction with the DØ Detector 29

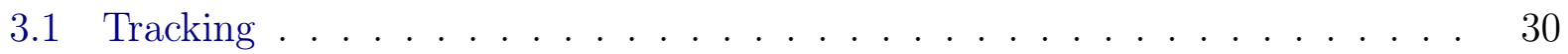

3.1.1 Layout of the Tracking Detectors . . . . . . . . . . . . . . . . 32

3.1.2 Track Reconstruction . . . . . . . . . . . . . . . . . 34

3.1.3 Vertex Reconstruction .................... 35

3.2 Calorimetry . . . . . . . . . . . . . . . . . . 37

3.2.1 Layout of the Calorimeter . . . . . . . . . . . . . . . . . 37

3.2.2 Cluster and Jet Reconstruction . . . . . . . . . . . . . . . . 39

3.3 Muon System . . . . . . . . . . . . . . . . . . . . . . 42

3.3.1 Layout of the Muon Detectors . . . . . . . . . . . . . . . . 43

3.3.2 Muon Reconstruction . . . . . . . . . . . . . . . . . . . . 44

3.4 Missing Energy . . . . . . . . . . . . . . . . . . . . . 46

3.4.1 Missing Energy Reconstruction . . . . . . . . . . . . . . . 46

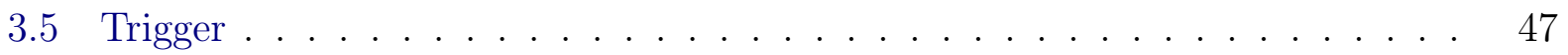

3.6 Event Simulation . . . . . . . . . . . . . . . . . . . 49

3.6.1 Overview of the Monte Carlo Tools . . . . . . . . . . . . . . . . 49

3.6.2 Usage of Monte Carlo Tools at D $\varnothing \ldots \ldots$. . . . . . . . . . 51 
4 Samples $\quad 53$

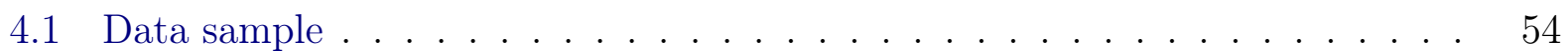

4.2 Monte Carlo Samples . . . . . . . . . . . . . . . . . . . . . . . . 55

4.2 .1 Signal Samples . . . . . . . . . . . . . . . . . 56

4.2 .2 Background Samples . . . . . . . . . . . . . . . . 57

4.3 Preselection . . . . . . . . . . . . . . . . . . . . . 58

5 Analysis $\quad 62$

5.1 Normalization . . . . . . . . . . . . . . . . . . . . 64

5.2 Multijet Estimate . . . . . . . . . . . . . . . . . 65

$5.3 \mathrm{MC}$ Corrections . . . . . . . . . . . . . . . . . 67

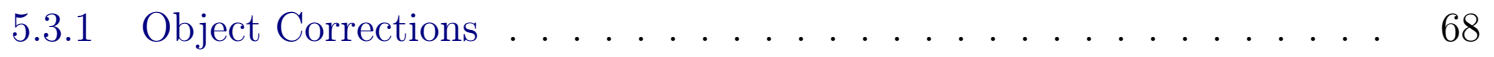

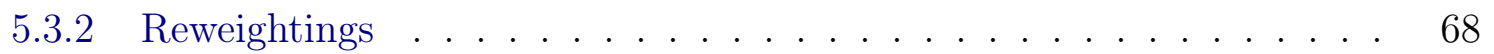

5.4 Multivariate Discriminant . . . . . . . . . . . . . . . . 71

5.4.1 Event Variables . . . . . . . . . . . . . . . . . . . 73

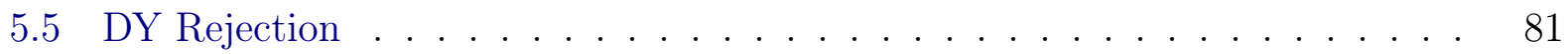

5.5.1 Cut-based DY Rejection .................... 81

5.5.2 Decision Tree DY Rejection ................ 82

5.6 Final Discriminant . . . . . . . . . . . . . . . . 86

6 Limit Calculation $\quad 90$

6.1 Statistical Treatment . . . . . . . . . . . . . . . . . 90

6.2 Systematic Uncertainties . . . . . . . . . . . . . . . . . . 94

6.3 Neural Network Results . . . . . . . . . . . . . . . . . . . 96

6.3.1 Standard Model Results . . . . . . . . . . . . . . . 96

6.3 .2 Fourth-Generation Interpretation . . . . . . . . . . . . . . . 99

6.4 Decision Trees Results . . . . . . . . . . . . . . . . . . . . 101

$\begin{array}{lll}7 \text { Conclusion } & 105\end{array}$ 
$\begin{array}{ll}\text { A Monte Carlo Samples and Cross Sections } & 108\end{array}$

A.1 Signal Samples and Cross Sections . . . . . . . . . . . . . . 108

A.2 Background Samples and Cross Sections . . . . . . . . . . . . . . 111

B Monte Carlo Reweightings $\quad 115$ 

ACKNOWLEDGMENTS

There are many exceptional people without whom I would not have been able to get to graduate school, and without whom completing this thesis would not have been possible.

My parents, Betti and Silvio, have always been the most admirable example of simplicity, hard work, and kindness. They are my gold standard for these qualities and I will always be grateful to them for supporting me over the years. My brothers, Giorgio, Matteo, Marco, and his wife Manuela, are the best family one could ever dream of: they are always there whenever I need anything, and spending time with them is always a pleasure. I also thank all my aunts, uncles, and cousins, who have always been very supportive. Special thanks are due to Mario and Rosalda, who have given me many rides when I traveled around the country throughout the years. Rosalda will be missed.

Growing up on a farm was a great way to stimulate my curiosity towards nature. Some exceptional teachers have turned this curiosity into a passion for learning. When I decided to attend the liceo scientifico, Maria Teresa Conte was the teacher who, although she was under no obligations to do so, generously spent time teaching me latin after school, so that I would survive my next educational stage. Hers was a rare example of dedication that I will never forget. Several teachers at the liceo scientifico "G.Ancina" taught me important lessons: Michelangela Bergese, Paolo Cortese, Attilio Ianniello, the late Mauro Re, the late Francesco Sampó, and Francesco Taricco.

When I was at the universitá di Torino, Professor Alessandra Romero gave me the opportunity to spend my first summer at CERN; that summer influenced my subsequent choices, and I thank professor Romero for believing in me then. Doctor Ernesto Migliore was also a great undergraduate advisor. He is both one of the smartest and most humble scholars I know. While they were not my offical advisors, Doctor Chiara Mariotti and Professor Michele Arneodo were extremely generous to me when I decided to apply to graduate school in the USA. I doubt that my applications would have been successfull without their support and precious suggestions.

Doctor Joe Haley welcomed me in Princeton when I came here as a prospective graduate student. I thank him for the help and suggestions over the past six years. Thanks are also due to Srevatsan Muralidharan, a great roommate during my first year in the graduate college, and a very good friend ever since. During my year at CERN in 2008, I spent a lot of time with Ted Laird. Sharing an apartment with Ted was a pleasure; sharing an office with him 
was stimulating; sharing the HPD and technical trigger work with him was always productive.

Professor Wade Fisher has been like a second advisor to me over the past three years. He got me started at $\mathrm{D} \varnothing$ with the tracking studies, and offered me to work on the $H \rightarrow W^{+} W^{-}$ search when I was looking for a thesis topic. His insightful comments and his witty humor have been essential to my research. I owe him a great debt of gratitude for being so generous with me.

I thank Professor Christopher Tully for taking me on as his advisee. Chris is the most alert and approachable physicist I know. He has always been there whenever I have asked for advice, and he has simultanously given me the freedom one needs to learn from one's own mistakes. He has supported all of my projects, and his enthusiasm was key to the success of many of them. Chris is the best advisor one could hope for, both academically and personally.

At Princeton, several people have helped me in many ways. For the fun and comradeship, I thank my fellow graduate students, including: Charles Mathy, Chris Laumann, Abhi Nellore, Justin Brown, and David Liao. For their company, I thank my office mates Adam Hunt, Andrzej Zuranskij, Tatiana Medvedeva, Halil Saka, and Mike Mooney. I am grateful to Stan Chidzik for always being nice and helpful any time I needed help fixing or modifying electronic equipment. Thanks are also due Elaine Remillard-Bridges and Kim Dawidowski, whose administrative help was invaluable. I am truly indebted to Eliza Zingesser, for being a very good friend, and for bravely agreeing to read several chapters of my thesis. While at Princeton, I was also fortunate to benefit from the expertise of many great professors, including Professor Bill Bialek and Professor Jim Olsen, whom I thank for agreeing to be on my dissertation committee.

This work would not have been possible without the support of the D $\varnothing$ Collaboration and the Fermi National Accelerator Laboratory. In particular, I would like to thank the following people. Dale Johnston and Mike Eads for teaching me the ropes of $H \rightarrow W^{+} W^{-}$; Wade Fisher, Aurelio Juste, Mike Kirby, and Marco Verzocchi for their numerous suggestions and comments on this study; my fellow analyzers Ruchika Nayyar, Kostas Petridis, and Jonas Weichert for being loyal, hard-working, and gifted colleagues and friends; Herb Greenlee, Mike Cooke, Alex Melnitchouk, and Zhenyu Ye for their help with the tracking software; Bill Lee and George Ginther for teaching me something about the detector every time I was in the control room, and for doing such a great job at running the experiment; Rick Vidal, Marco Verzocchi, Wade Fisher, and Stefan Soldner-Rembold for supporting my fellowship and post-doc applications.

Lastly, I want to thank my partner Eve, without whom my life at Princeton wouldn't have been the same. Her constant support was essential to this thesis and, in general, to my sanity over the last six years. Her strength and tenacity (and appetite for good food) are things I wasn't expecting from this tiny mademoiselle...Merci! 
Ci sono molte persone senza le quali non sarei arrivato al dottorato e senza cui questa tesi non sarebbe stata possibile.

I miei genitori, Betti e Silvio, sono sempre stati per me un esempio ammirabile di semplicitá, lavoro sodo, e gentilezza. Quando penso a loro, spesso mi meraviglio ancora di quanto si possa fare con queste tre qualitá, e saró loro sempre riconoscente per il sostegno datomi da sempre. I miei fratelli Giorgio, Matteo e Marco con la sua moglie Manuela, sono la famiglia migliore che chiunque possa mai sognarsi: ci sono sempre ogni volta che io ne abbia bisogno, e passare il tempo con loro é sempre una gioia. Ringrazio anche tutte le zie, zii, cugine e cugini che mi hanno sempre sostenuto; in particolare ringrazio Mario e Rosalda, che erano sempre pronti a scarrozzarmi in giro per i miei viaggi. Rosalda ci mancherá tanto.

Crescere in campagna é stato un ottimo modo per stimolare la mia curiositá per la natura. Alcuni insegnanti eccezionali hanno trasformato questa curiositá in passione per l'apprendimento. Quando decisi di iscrivermi al liceo scientifico, Maria Teresa Conte e' stata l'insegnante che, senza alcuna obbligazione e senza ricompensa monetaria, spese parte del suo tempo doposcuola insegnandomi latino affinché il mio passaggio al liceo fosse meno difficile. Il suo é stato per me un raro esempio di dedizione che non dimenticheró. Numerosi insegnanti al liceo scientifico "G. Ancina" mi hanno dato lezioni importanti; tra loro: Michelangela Bergese, Paolo Cortese, Attilio Ianniello, il compianto Mauro Re, il compianto Francesco Sampó e Francesco Taricco.

All'universitá di Torino, la Professoressa Alessandra Romero mi diede l'opportunitá di passare al CERN la mia prima estate; quella estate ha influenzato le mie scelte successive, e ringrazio Alessandra Romero per aver creduto in me a quel punto. Il Dottor Ernesto Migliore é stato un ottimo relatore; é anche uno degli studiosi piú intelligenti che io conosca, ed allo stesso tempo il piú modesto. Nonostante non fossero formalmente i miei relatori, la Dottoressa Chiara Mariotti ed il Professor Michele Arneodo sono stati con me estremamente generosi quando decisi di fare domanda negli USA per il dottorato; indubbiamente the mie domande non sarebbero andate cosí bene senza i loro preziosi consigli.

Il Dottor Joe Haley mi ospitó quando visitai Princeton come futuro studente; lo ringrazio per l'aiuto ed i consigli datimi durante questi sei anni. Srevatsan Muralidharan é stato un ottimo compagno di stanza durante il mio primo anno nel graduate college, ed un ottimo amico di lí in poi. Durante l'anno 2008 che ho passato al CERN, ho condiviso un sacco di 
tempo con Ted Laird. Condividere un appartamento con Ted é stato divertente; condividere un ufficio con lui é stato produttivo e stimolante; condividere il lavoro sugli HPD e sul technical trigger é stato un piacere. Il Professor Wade Fisher é stato per me come un secondo relatore. É stato lui ad avviarmi con lo studio a DØ sul tracciamento, e successivamente ad offrirmi di lavorare sulla ricerca $H \rightarrow W^{+} W^{-}$quando cercavo un argomento di tesi. I suoi attenti suggerimenti in fisica ed le sue battute argute sono stati essenziali per il mio lavoro, e debbo a Wade molta riconoscenza per essere stato cosí generoso con me.

Ringrazio il mio relatore, Professor Christopher Tully, per avermi preso come dottorando. Chris é il fisico piú sveglio che io conosca, ed é sempre alla buona con i suoi studenti. Mi ha lasciato quella libertá di cui uno ha bisogno per imparare dai propri sbagli; allo stesso tempo é stato sempre presente ogni volta che avessi bisogno di consigli. Mi ha sempre sostenuto nei miei progetti, ed il suo entusiasmo é stato essenziale per il successo di molti. Chris é il miglior relatore che potessi mai immaginare, sia dal punto di vista accademico che umano.

Molte persone mi hanno aiutato a Princeton in un modo o nell'altro. I miei compagni di corso sono stati fantastici. Per il divertimento e la compagnia Charles Mathy, Chris Laumann, Abhi Nellore, Justin Brown e David Liao. I miei compagni di ufficio: Adam Hunt, Andrzej Zuranskij, Tatiana Medvedeva, Halil Saka, e Mike Mooney. Ogni volta che ho avuto bisogno di aggiustare o modificare dell'elettronica, Stan Chidzik é sempre stato molto gentile e utile. Di grandissimo aiuto nelle cose amministrative sono state Elaine Remillard-Bridges e Kim Dawidowski. Ringrazio di cuore Eliza Zingesser, per essere un'ottima amica, e per aver letto molti capitoli di questa mia tesi. In Princeton ho avuto la fortuna di interagire con ottimi professori. Tra questi, ringrazio il Professor Bill Bialek ed il Professor Jim Olsen per accettare di far parte della mia commissione d'esame.

Questo lavoro non sarebbe stato possibile senza la Collaborazione D $\varnothing$ e senza il Fermilab. Vorrei ringraziare in particolare le seguenti persone. Dale Johnston e Mike Eads per avermi insegnato i rudimenti di $H \rightarrow W^{+} W^{-}$. Wade Fisher, Aurelio Juste, Mike Kirby e Marco Verzocchi per i loro numerosi suggerimenti e commenti riguardo questo studio. I miei compagni di analisi Ruchika Nayyar, Kostas Petridis, e Jonas Weichert per essere stati collaboratori ed amici leali, impegnati e capaci. Herb Greenlee, Mike Cooke, Alex Melnitchouk, e Zhenyu Ye per l'aiuto con il codice di tracciamento. Bill Lee e George Ginther, per avermi insegnato qualcosa del rivelatore ogni volta che ero in sala di controllo, e per il loro impegno eccezionale a mantenere l'esperimento. Rick Vidal, Marco Verzocchi, Wade Fisher e Stefan SoldnerRembold per avermi aiutato nelle mie domande per borse e post-doc.

Infine voglio ringraziare la mia compagna Eve, senza la quale la mia vita a Princeton non sarebbe stata la stessa. Il suo continuo sostegno é stato essenziale per questa tesi ed, in generale, per la mia sopravvivenza durante gli ultimi cinque anni. Da questa piccola mademoiselle non mi aspettavo tale forza e tenacia (ed appetito per il buon cibo!)...Merci! 


\section{LIST OF ABBREVIATIONS}

$\begin{array}{ll}\text { CL } & \text { Confidence Level } \\ \text { CC } & \text { Central Calorimeter } \\ \text { CFT } & \text { Central Fiber Tracker } \\ \text { CH } & \text { Coarse Hadronic } \\ \text { CPS } & \text { Central Preshower } \\ \text { CPS } & \text { Forward Preshower } \\ \text { DCA } & \text { Distance of Closest Approach } \\ \text { DQ } & \text { Data Quality } \\ \text { DY } & \text { Drell-Yan } \\ \text { DY DT } & \text { Drell-Yan decision tree } \\ \text { EC } & \text { End Calorimeter } \\ \text { EM } & \text { Electromagnetic }\end{array}$

FAMUS Forward Angle Muon System

FD DT Final discriminant decision tree

Fermilab Fermi National Accelerator Laboratory

FH Fine Hadronic

FNAL Fermi National Accelerator Laboratory

HP Hard Process 
ICD Inter-Cryostat Detector

JES Jet Energy Scale

JSSR Jet Shifting, Smearing, and Removal

LeBOb Low Energy Background Object

LINAC Linear accelerator

LLR Log-Likelihood Ratio

MC Monte Carlo

MET Missing Transverse Energy

PDF Parton Distribution Function

PV Primary Vertex

QCD Quantum Chromodynamics

RF Radio Frequency

SM Standard Model

SMT Silicon Microstrip Tracker

UE Unclustered Energy

WAMUS Worward Angle Muon System 


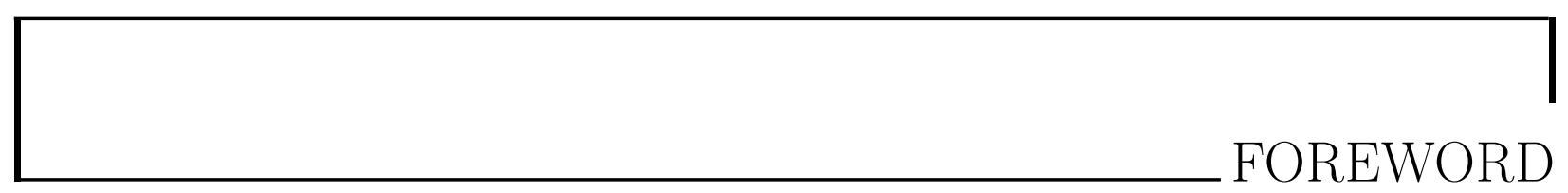

The Higgs boson is the chief missing piece in our current understanding of fundamental particles. What is usually called "the search for the Higgs boson" is, in fact, the way experimentalists try to answer the following two questions: is the standard model consistent? how do particles acquire a mass? This thesis describes a search for the Higgs boson performed by studing $\mathrm{p} \overline{\mathrm{p}}$ collisions events with two leptons and transverse energy imbalance. Although this is one search within a decades-long quest, the result of this analysis represents a milestone, because it provides a clear answer for a heavy Higgs boson-albeit a negative one.

The first two chapters give an overview of the theoretical and experimental landscape within which this search was performed; the following ones describe the analysis which is the subject of this thesis. Chapter one provides a brief sumamry of the standard model and of the Higgs boson. Chapter two illustrates the main features of hadron collider experiments: particle acceleration and particle detection. Chapter three illustrates the $\mathrm{D} \varnothing$ detector, how it is used to record $\mathrm{p} \overline{\mathrm{p}}$ collisions, and how physics objects are recontructed within an event. Chapter four describes the event samples and their selection. Chapter five describes the data analysis. The search results are reported in chapter six.

While the analysis description given here focuses on the final state with two muons $\left(\mu^{+} \mu^{-}\right)$, the analysis tools are the same ones used to analyze the final states with $\mathrm{e}^{+} \mathrm{e}^{-}$and $\mathrm{e}^{ \pm} \mu^{\mp}$; the results obtained combining these three channels are also discussed. 


\section{CHAPTER 1}

This chapter sets out to frame the search for the Higgs boson. After illustrating the motivations for the work described in this thesis, a brief overview of the standard model of particle physics is given, followed by a description of the Higgs boson's properties.

\subsection{Motivation}

One of the greatest achievements of modern science is the explanation of matter in terms of fundamental constituents. Although the definition of what we call "fundamental" has changed over time ${ }^{1}$, the basic principle has remained the same: the description of all matter as a combination of a few elementary building blocks. The standard model (SM) of particle physics is a theoretical framework that explains all matter with twelve elementary constituents.

\subsubsection{Construction and Limitations of the Standard Model}

In the same way that the periodic table describes all chemical elements in terms of protons, neutrons, and electrons, the SM describes all known matter as made of six leptons and six

\footnotetext{
${ }^{1}$ Particles that, at some point in history, are thought to be fundamental can be described, later on, as composite objects made of smaller entities. For example, this happened with atoms and then with protons and neutrons which, in the past, were thought to be the building blocks of matter.
} 
quarks. These fundamental particles interact by exchanging force mediators, which correspond to three forces: electromagnetic, weak, and strong. This description is valid at very small distances, typically smaller than the atomic nucleus $\left(10^{-15} \mathrm{~m}\right)$. Gravitiy is not included in this picture: since it is much weaker ${ }^{2}$ than the other three forces, its effects are negligible for subatomic particles.

The SM was built over the last five decades, and its predictions have been tested by many experiments (see Ref. [1] for a review, and Ref. [2] for a more detailed description). So far, all of the experimental observations are in agreement ${ }^{3}$ with the SM predictions. Despite this success, the SM has one major shortcoming: it does not include mass terms. In other words, all of the SM equations remain valid if we assume that all particles have a mass equal to zero. Furthermore, the addition of ad hoc mass terms does not resolve this issue. Such terms make the model inconsistent and useless: they lead to the prediction of probabilities greater than one, which are not mathematically possible.

In 1964, a solution was proposed by Higgs [3], Englert [4], Guralnik [5], and collaborators. They suggested that the model can admit mass terms, while still being consistent, if those are generated by the interaction with a constant scalar field permeating all space. If this field exists, we should be able to observe the corresponding excitation: a spin-0 particle named the "Higgs boson". The Higgs boson is the last missing piece of the SM: it has not been observed yet in any experiment. While its observation would ultimately confirm our understanding of fundamental particles, its exclusion would substantiate the idea that our model is an approximation of reality - albeit a good one.

In the past, unsuccessful searches for the Higgs boson have been performed at several collider experiments. These searches [6] were sensitive to a Higgs boson with a mass of, at most, $0.115 \mathrm{TeV}$. Hypotheses with a heavier Higgs boson could not be tested, due to the

\footnotetext{
${ }^{2}$ For example, in a hydrogen atom, the attraction between the electron and the proton due to gravity is almost 40 orders of magnitude smaller than the one due to the electromagnetic force.

${ }^{3}$ This is true to the extent that the predictions of the theory can be calculated. When it is not possible to compute from first priciples the quantities that can be measured in experiments, it is often necessary to resort to phenomenological models which, strictly speaking, are not part of the SM.
} 
limited energy of the colliding particle beams.

Nevertheless, global fits [7] of the SM parameters to the data from previous experiments indicate that, if the Higgs boson exists, it is most likely to be found in the mass range from $0.1 \mathrm{TeV}$ to $0.2 \mathrm{TeV}$. Thus, in order to validate our understanding of fundamental particles and forces, it is important to search for the Higgs boson in this mass range.

\subsubsection{Why Search for the Higgs Boson at the Tevatron}

The Tevatron collider at Fermilab (Batavia, IL) is one of the two places in the world - the other being the Large Hadron Collider in Geneva, Switzerland-where particles can collide at energies that could be sufficient to produce and observe the Higgs boson. At the Tevatron, the proton beam collides with the antiproton beam with a center of mass energy of $1.96 \mathrm{TeV}$; this energy allows us to search for a Higgs boson as heavy as $0.2 \mathrm{TeV}$. Unfortunately, the production of a Higgs boson is an extremely rare process, and a large number of collisions is necessary for its observation. After collecting data for several years, the two experiments at the Tevatron, $\mathrm{CDF}$ and $\mathrm{D} \varnothing$, have been able to accumulate a dataset that is large enough to observe such rare processes.

In order to determine whether the Higgs boson exists, it is necessary to extract a small number of signal-like events from a large dataset dominated by background events. This is done by identifying characteristic features of those signal-like events. As pointed out by Han et al. [8], one hypothesis in which such features are particularly promising is the one where the Higgs boson is heavy, at least twice as heavy as the $\mathrm{W}$ boson. In this case, the Higgs is expected to decay most of the time to a WW pair, $\mathrm{H} \rightarrow \mathrm{WW}$. In turn, about one out of three times, each $\mathrm{W}$ decays to a charged lepton and a neutrino, $\mathrm{W} \rightarrow \ell \nu$. Leptons can be efficiently identified with the detector, and the neutrinos' presence can be inferred from the

imbalance in the transverse energy. Thus, the decay chain $\mathrm{H} \rightarrow \mathrm{W}^{+} \mathrm{W}^{-} \rightarrow \bar{\ell} \nu \ell \bar{\nu}$ leads to a final state with a clear signature, which can be used to identify candidate Higgs events in a hadron collider experiment. 
Over the last decade, experimenters at the Tevatron have pursued this strategy and have improved the required data analysis techniques. At the same time, theorists have made great progress calculating, with increasing precision, the cross section and the kinematic features of the Higgs production processes.

These experimental and theoretical advances, along with the large number of $\mathrm{p} \overline{\mathrm{p}}$ collisions recorded during the Tevatron RunII, allowed us to reach the sensitivity needed to test the Higgs boson hypothesis, at least in part of the $0.1-0.2 \mathrm{TeV}$ mass range. The work described in this thesis excluded, for the first time at a hadron collider, a Higgs boson with a hypotetical mass of about $165 \mathrm{GeV}$. The exclusion was first achieved [9] combining the two $H \rightarrow W^{+} W^{-}$ searches by $\mathrm{CDF}$ and $\mathrm{D} \varnothing$ and, more recently, with the $\mathrm{D} \varnothing$ dataset alone [10]. These are two major milestones: although the hypothesis of a Higgs boson still needs to be tested for the mass range left unprobed, these results indicate that hadron collider experiments will soon be able to determine whether the Higgs boson is the solution to the standard model inconsistency. If not, the model will need more radical changes.

\subsection{The Standard Model}

The standard model (SM) describes elementary particles combining the two theories of special relativity and quantum mechanics. All particles are interpreted as excitations of relativistic quantum fields, and their behavior is determined by the SM Lagrangian, which is a function of these fields. Quantum fields can be fermionic (with half-integer spin) or bosonic (with integer spin). The corresponding field excitations are called fermions and bosons.

\subsubsection{Subatomic Matter and Forces}

Subatomic matter is made of spin- $1 / 2$ fermions, each having an anti-particle with opposite electric charge. Fermions are organized in three generations: those belonging to the same generation have similar properties, namely they have the same flavor, but their masses 
increase from one generation to the next. Each generation contains two quarks and two leptons, for a total of twelve fundamental constituents of matter.

Quarks and leptons interact by exchanging spin-1 bosons, which are responsible for the electromagnetic, weak, and strong forces. A schematic view of the standard model particles and interactions is shown in figure 1.1.

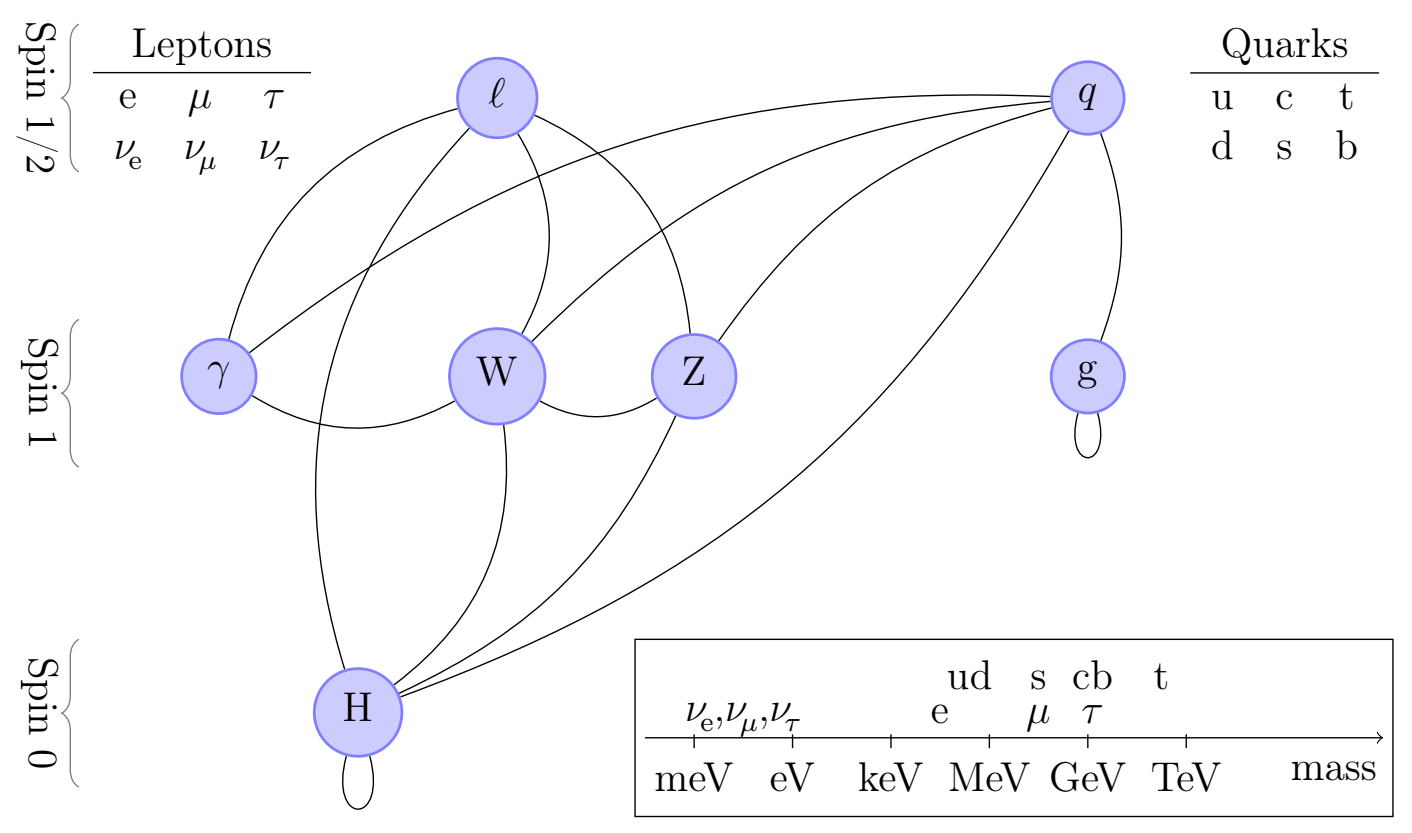

Figure 1.1: Schematic view of the Standard Model particles and of their interactions. Connecting lines represent interactions between particles. The masses of the fundamental constituents are indicated in the bottom-right inset. In this picture, the Higgs boson is the only particle that has not yet been observed. Redrawn from Ref. [11].

The electromagnetic force affects all particles with electric charge. It is mediated by photons and it has infinite range. This force has been known for a long time, and the other two forces are named "weak" and "strong" after their relative strengths in comparison to the electromagnetic force.

The strong force, or quantum chromodynamics (QCD), is mediated by gluons and acts on quarks, which carry a color charge. QCD holds together the two quarks that make up a meson, and the three quarks that make up a barion, such as protons and neutrons. QCD has two peculiar features, setting it apart from the other known forces. First, gluons carry a color 
charge; therefore, they interact not only with the quarks, but also with themselves. Second, the strong coupling constant $\alpha_{s}$ becomes weaker at higher energies. These two properties have the important consequence that quarks cannot be observed in isolation, an effect called quark confinement. In large part, the binding energy of quarks confined inside protons and neutrons accounts for the mass of these barions. Therefore, most of the visible mass in the universe arises from QCD.

The weak force affects all fermions and is mediated by the $\mathrm{W}^{ \pm}$and $\mathrm{Z}^{0}$ bosons. Beside being weak, as its name indicates, this force has three peculiar properties. First, it differentiates between left and right ${ }^{4}$ fermions. Second, it can change the flavor of quarks. Third, it has a short range, less than $10^{-16} \mathrm{~m}$. The reason for such a short range is that the $\mathrm{W}$ and $\mathrm{Z}$ are massive bosons. Therefore, they can transmit the weak force over a limited distance, unlike the photon which is massless and can transmit the electromagnetic force over an infinite range. In fact, despite looking so different to us, the electromagnetic and weak interactions are one single entity, the electroweak interaction. The electromagnetic and weak forces originate in the same way from a common symmetry, and "the weak force appears to be so feeble only because of its limited range, but not because of its intrinsic strength."[12]

The generation of the $\mathrm{W}$ and $\mathrm{Z}$ masses, along with the differentiation of the electroweak force in the electromagnetic and weak interactions, is the main reason for which a Higgs boson is introduced. The concept behind all SM interactions, the idea of gauge symmetry, is briefly described in section 1.2.2, followed by a description of the Higgs mechanism in section 1.3 .

\subsubsection{Gauge Symmetries}

The Lagrangian of a physical system is said to be gauge invariant, or equivalently to have a gauge symmetry, if it remains constant under a continuous gauge (phase) transformation. Gauge symmetries of the Lagrangian are the fundamental concept behind the SM, for

\footnotetext{
${ }^{4}$ We call right-handed (or simply right) a fermion whose spin and momentum are parallel, and left-handed (or left) a fermion for which they are anti-parallel.
} 
the interactions between particles are completely determined once the symmetry has been specified.

Gauge symmetries can not only describe the three SM interactions; they also allow for the unification of the electromagnetic and weak forces into one electroweak interaction, which comes into being because of the $\mathrm{SU}(2)_{L} \times \mathrm{U}(1)_{Y}$ symmetry of the Lagrangian. This unification, proposed in 1968 by Glashow [13], Weinberg [14], and Salam [15], led to the prediction of the $\mathrm{W}$ and $\mathrm{Z}$ bosons, the mediators of the weak force that were discovered in 1983.

These very same powerful concepts, which can unify forces and predict the existence of unknown particles, are at the origin of three inconsistencies within the SM. First, they do not differentiate between left and right leptons. Second, they treat charged leptons and neutrinos on an equal footing, even though they have different mass and electric charge. Third, they require that the bosons mediating the weak force be massless. These three shortcomings can be addressed with the Higgs mechanism, which implements in its simplest form the spontaneous symmetry breaking of the electroweak sector of the SM.

\subsection{The Higgs Boson}

The Higgs mechanism is the simplest known way to generate the masses $m_{W}$ and $m_{Z}$ for the weak force mediators $\mathrm{W}$ and $\mathrm{Z}$ while maintaining the renormalizability of the SM. In other words, this mechanism can fix the inconsistencies arising within the electroweak sector of the SM, and at the same time avoids incurring the divergent results that often arise within quantum field theories.

\subsubsection{Symmetry Breaking and the Higgs Boson}

Spontaneous symmetry breaking occurs when the ground state of the system is not invariant under the transformation which defines the symmetry of the Lagrangian. In the SM case, the electroweak symmetry must be hidden, or broken, "recognizing that a symmetry of the 
laws of Nature does not imply the same symmetry in the outcome of those laws."[16] This can be achieved with the introduction of a self-interacting scalar field, the Higgs field, with a non-zero vacuum expectation value $v$. In its simplest form, the Higgs mechanism postulates the existence of a scalar field that is a complex doublet $\Phi$ with the following potential:

$$
V(\Phi)=-\mu^{2} \Phi^{\dagger} \Phi+\lambda\left(\Phi^{\dagger} \Phi\right)^{2}
$$

where $\mu^{2}>0$ and $\lambda>0$. The vacuum expectation value of the potential is determined from existing measurements. This constraint fixes one of the two parameters, leaving the other free. By expanding in power series the potential near the minimum, one can see that excitations around the ground state have a quadratic term, that is a mass term, with the free parameter as the coefficient. In other words, the mass of the Higgs boson $m_{H}$ is not specified by the theory.

\subsubsection{Properties of the Higgs Boson}

Several properties of the Higgs boson are predicted by the theory. Furthermore, constraints on its unknown mass can be determined from both theoretical assuptions and experimental measurements.

Theoretical and Experimental Constraints on $\mathbf{m}_{\mathbf{H}}$ The Higgs mechanism is valid only within some theoretical assumptions. As mentioned in the previous paragraph, the mass of the Higgs boson is strictly related to the curvature of the potential near its minimum. On the one hand, if the curvature becomes too big the theory breaks down because it can no longer be treated perturbatively. On the other hand, if the curvature is too small, the local minimum of the potential disappears, and the ground state is symmetric again. These two effects, along with more advanced ones such as the WW scattering amplitude, provide theoretical lower and upper bounds to the possible values of $\mathrm{m}_{\mathrm{H}}$.

Loop diagrams such as the ones shown in figure 1.2 give a precise relation between the 

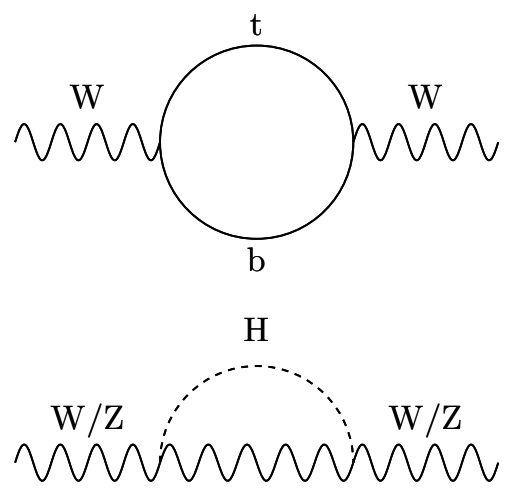

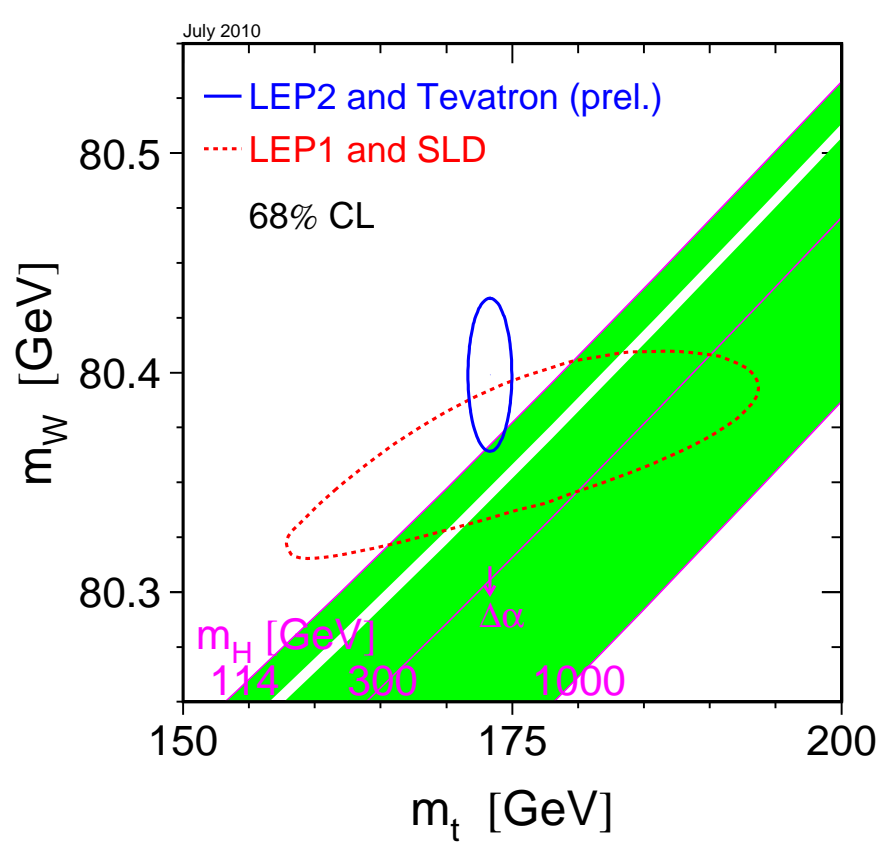

Figure 1.2: The masses $\mathrm{m}_{\mathrm{H}}, \mathrm{m}_{\mathrm{W}}$, and $\mathrm{m}_{\mathrm{t}}$ are related through loop diagrams, such as the ones shown on the left. The plot on the right compares the constraints on $\mathrm{m}_{\mathrm{W}}$ and $M_{\mathrm{T}}$ : the indirect constraints, due to loop diagrams, are indicated with the dashed 68\% CL countour; the direct constraints, from LEP and Tevatron measurements of $\mathrm{m}_{\mathrm{W}}$ and $M_{\mathrm{T}}$, are indicated with the solid $68 \%$ CL countour. Higgs masses are also shown on this plane. From Ref. [7].

mass of the top quark, the $\mathrm{W}$ boson and the Higgs boson. Such a relation, along with others, is used to combine the electroweak observables in a global fit to the electroweak measurements that were performed in past experiments. The goodness of this fit, shown in figure 1.3, provides an indirect experimental indication on the most likely value of $\mathrm{m}_{\mathrm{H}}$, suggesting the mass range $0.1-0.2 \mathrm{GeV}$ as the most likely one for a SM Higgs boson.

Direct constraints on the value of $\mathrm{m}_{\mathrm{H}}$ come from past searches for the Higgs boson. The results from the Higgs searches performed at the LEP collider, described in Ref. [17], indicate that, at $95 \%$ confidence level, the Higgs boson should be heavier than $115 \mathrm{GeV}$. The LEP result was, before the Tevatron results described in this thesis, the most stringent constraint for a Higgs boson with a hypotetical mass in the range $0.1-0.2 \mathrm{GeV}$. 


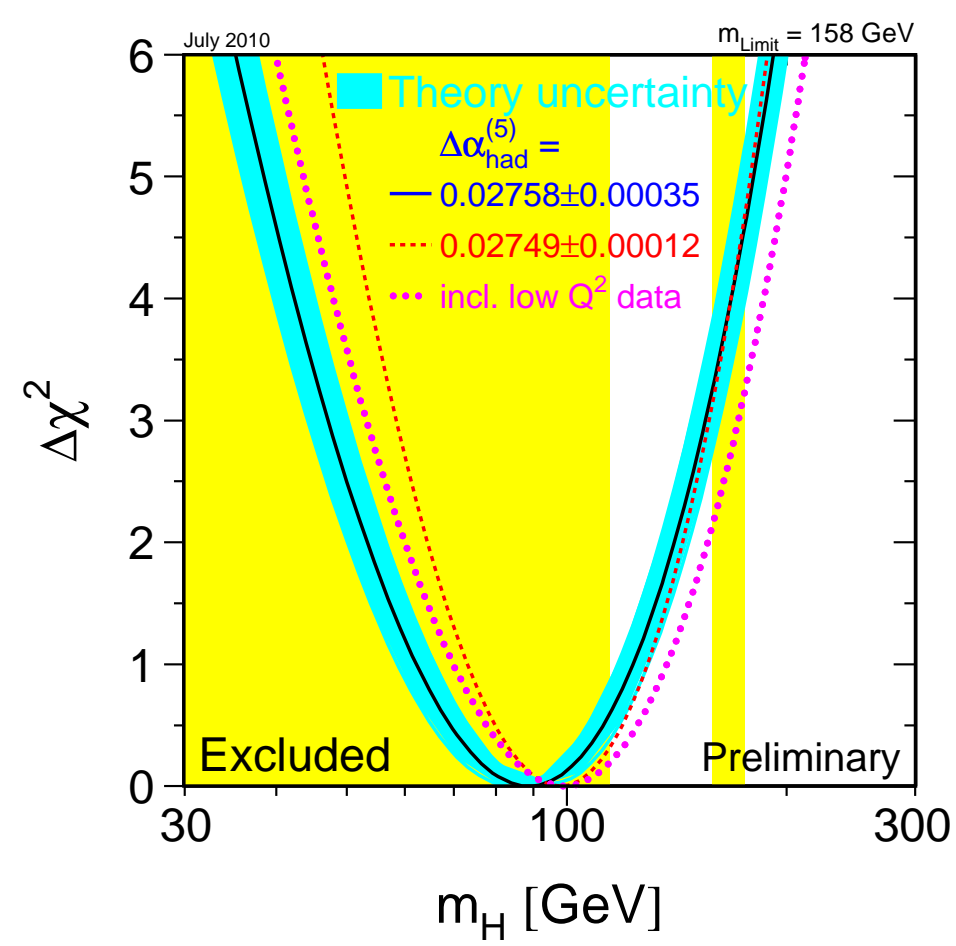

Figure 1.3: $\Delta \chi^{2}$ as a function of $\mathrm{m}_{\mathrm{H}}$ for the global fit to the electroweak measurements performed by several experiments, from [7]. The most likely $\mathrm{m}_{\mathrm{H}}$ value corresponds to the minimum $\Delta \chi^{2}$, with the theoretical uncertainty indicated by the blue band. The two yellow vertical bands indicate the two mass ranges that have been escluded by direct experimental searches. The left yellow band corresponds to the LEP exclusion. The right yellow band corresponds to the most recent Tevatron exclusion.

Couplings of the Higgs Boson The couplings of the Higgs boson to the other SM particles are specified by the theory, allowing for the calculation of the production cross section and of the branching ratios. The Higgs boson couples directly to all massive particles: its coupling to fermions is linear in the mass of the fermion, and its coupling to weak bosons is quadratic in the weak boson mass. Indirect couplings to massless bosons (photons and gluons) are also possible: at leading order, they arise from Feynman diagrams having one intermediate loop with a massive particle. As a consequence of these coupling rules, in general, the diagrams that contibute the most to the production or decay of the Higgs boson are those involving weak bosons or heavy fermions.

Higgs Boson Production Several mechanisms can lead to the production of a Higgs boson; the corresponding cross sections are shown, as a function of $\mathrm{m}_{\mathrm{H}}$, in figure 1.4. The mechanism with the largest cross section is the gluon-gluon fusion process, whose corresponding Feynman diagram is shown in figure 1.5(a). In this process, two gluons couple to the Higgs boson 
through an intermediate fermion loop; this diagram contributes the most when the fermion in the loop is heavy, for example a third generation quark such as the top. Should a fourth generation of heavier quarks exist, it would provide additional contributions to this process, and increase the gluon-gluon fusion cross section by almost an order of magnitude, as shown in figure 1.6.

The production mechanism with the second largest cross section is the associated production, whose corresponding Feynman diagram is shown in figure 1.5(b). In this process, two quarks annihilate each other to produce an off-shell weak boson $\left(\right.$ a $\mathrm{W}^{ \pm}$or a $\left.\mathrm{Z}^{0}\right)$, which then emits a Higgs boson. The associated production is usually indicated with VH, as an abbreviation for $\mathrm{WH}$ or $\mathrm{ZH}$.

The production mechanism with the third largest cross section is the vector boson fusion, shown in figure 1.5(c). In this process, the two incoming quarks emit $\mathrm{W}$ or $\mathrm{Z}$ bosons, which combine to form a Higgs boson. The experimental signature of this channel includes, beside the particles from the Higgs decay, two forward jets due to the two scattering quarks.
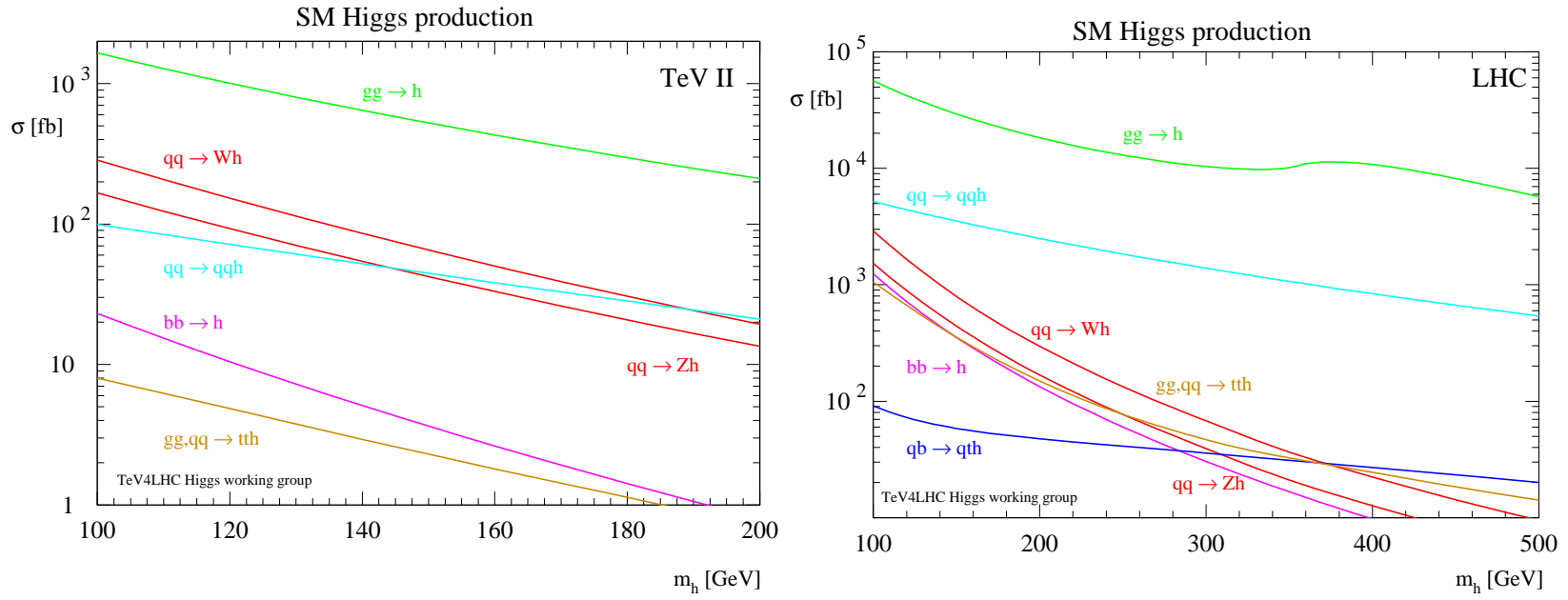

Figure 1.4: Expected cross sections of the most important Higgs production processes at the Tevatron (left) and at the LHC (right), as a function of the Higgs boson. From the Tevatron for LHC report [18].

Higgs Boson Decay The Higgs boson couplings are stronger for heavy bosons and leptons. Thus, it decays primarily to the heaviest particles that are kinematically available. The 


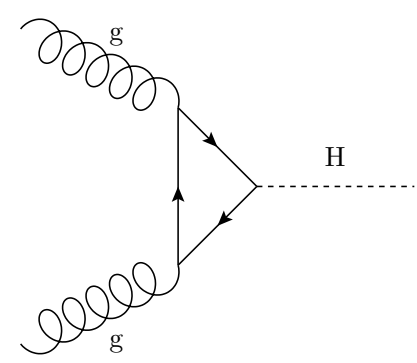

(a)

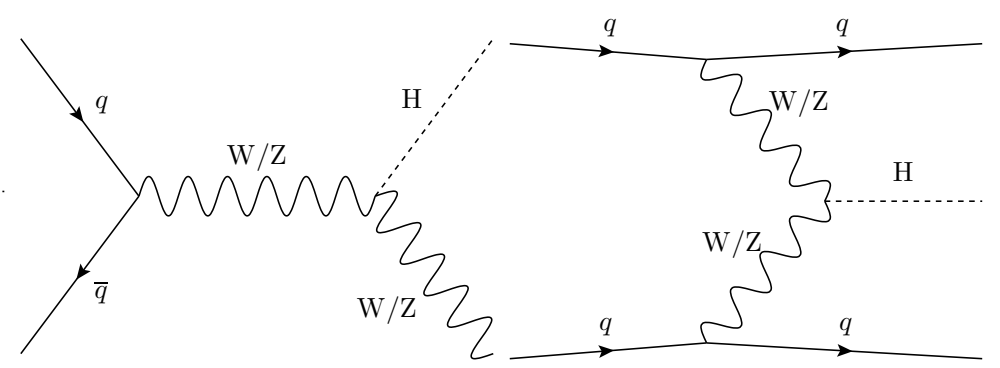

(b)

(c)

Figure 1.5: Feynman diagrams for the three most important production mechanisms at the Tevatron: (a) gluon-gluon fusion, (b) associated production, and (c) vector boson fusion.

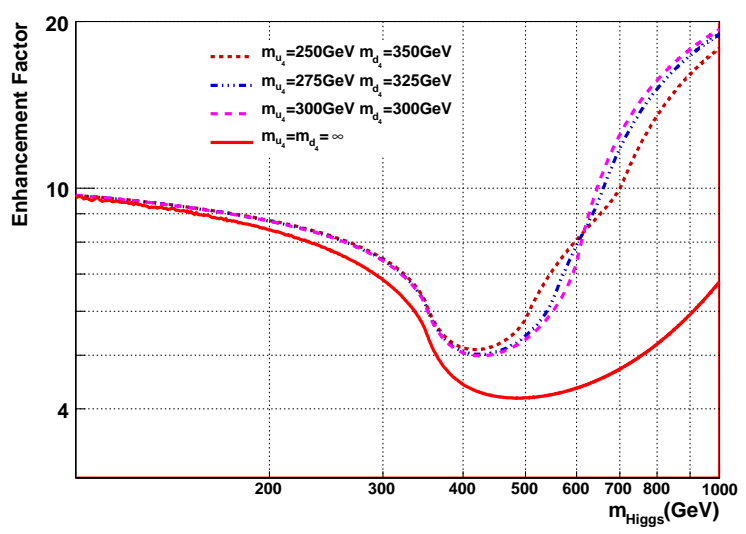

Figure 1.6: Enhancement factor for the $\mathrm{gg} \rightarrow \mathrm{H}$ cross section under the assumption that a fourth SM quark family exists. The contributions due to additional quark loops vary as a function of the hypothetical quarks' mass. From Ref. [19].

branching ratios of the Higgs boson are shown in figure 1.7 as a function of $\mathrm{m}_{\mathrm{H}}$.

For $\mathrm{m}_{\mathrm{H}}<135 \mathrm{GeV}$, the SM predicts that the Higgs boson decays most of the time to $b \bar{b}$. More rarely, less than $10 \%$ of the time, it can decay to $\tau \tau$ and $c \bar{c}$. The probability for a Higgs boson to decay to two photons is even smaller, below $0.3 \%$. Nevertheless, because of the low background for the diphoton final state, this decay provides an important channel to search for a Higgs boson with $115<\mathrm{m}_{\mathrm{H}}<135 \mathrm{GeV}$.

A Higgs boson with a mass greater than $135 \mathrm{GeV}$ can decay to a pair of weak bosons, one of which is usually off-shell. In the mass region $135<\mathrm{m}_{\mathrm{H}}<200 \mathrm{GeV}, H \rightarrow W^{+} W^{-}$is the dominant decay channel. For $\mathrm{m}_{\mathrm{H}}$ values above $180 \mathrm{GeV}$, the final state with a $\mathrm{Z}$ pair becomes available, and the Higgs boson decays $H \rightarrow \mathrm{ZZ}$ more than $20 \%$ of the time. 


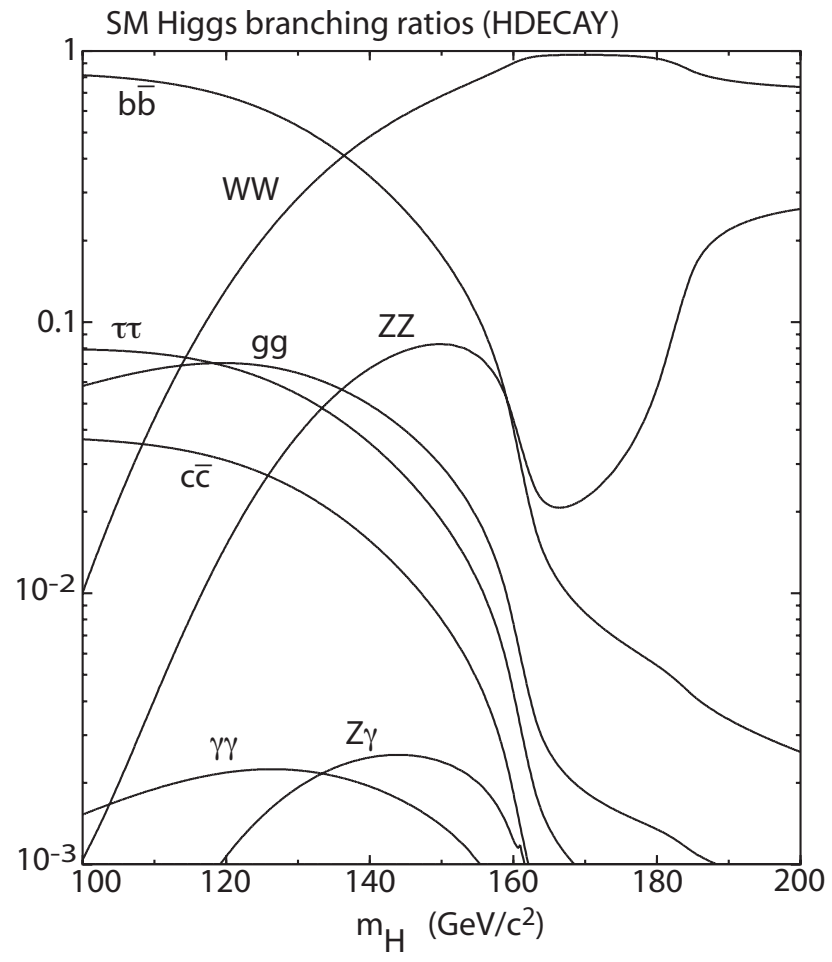

Figure 1.7: Branching fractions for a minimal standard model Higgs boson, as computed by HDECAY [20]. The two dominant decays, $H \rightarrow b \bar{b}$ and $H \rightarrow W^{+} W^{-}$, cross over at $\mathrm{m}_{\mathrm{H}} \simeq 135 \mathrm{GeV}$, defining the lowmass and the high-mass regions. The remanining channels account at most for $10 \%$ of the decays.

\subsubsection{Strategy for a Higgs Boson Search}

Current searches for a Higgs boson fall in two major categories: low-mass ones and high-mass ones. Low-mass searches, which are optimized for a Higgs boson with $\mathrm{m}_{\mathrm{H}} \lesssim 135 \mathrm{GeV}$, focus on the $H \rightarrow b \bar{b}$ decay. High-mass searches, which are optimized for a Higgs boson with $\mathrm{m}_{\mathrm{H}} \gtrsim 135 \mathrm{GeV}$, focus on the $H \rightarrow W^{+} W^{-}$and $H \rightarrow \mathrm{ZZ}$ decays.

Low Mass Searches For $\mathrm{m}_{\mathrm{H}} \lesssim 135 \mathrm{GeV}$, the study of the gg $\rightarrow H \rightarrow b \bar{b}$ process is not viable because it suffers from large backgrounds due to non-resonant $b \bar{b}$ production. Instead, low mass searches rely on the associated production of the Higgs boson. High- $p_{\mathrm{T}}$ leptons and large $E_{\mathrm{T}}$ are both signatures of the weak boson production that can be used as a trigger in $\mathrm{VH}$ events, and allow for the offline analysis of the two $b$-jets from $H \rightarrow b \bar{b}$. Despite the advantages brought by this triggering technique, $\mathrm{VH}(H \rightarrow b \bar{b})$ searches are challenging because of the relatively low $\mathrm{VH}$ cross section. This search is even more challenging at the LHC, where the signal-to-noise ratio is worse than the one at the Tevatron, and more advanced techniques are needed, such as selecting boosted $H \rightarrow b \bar{b}$ decays. In addition to 
$H \rightarrow b \bar{b}$, low mass searches can use the $H \rightarrow \gamma \gamma$ decay. Unfortunately, the corresponding branching fraction is at most $0.3 \%$, requiring the collection of a large datateset. In addition, a highly optimized photon detection is needed, which might be available with the LHC detectors, but would be limited with the Tevatron ones.

High Mass Searches For $\mathrm{m}_{\mathrm{H}} \gtrsim 135 \mathrm{GeV}$, Higgs searches rely on the $H \rightarrow W^{+} W^{-}$decay and, to some extent, on that of $H \rightarrow \mathrm{ZZ}$, which becomes more relevant for $\mathrm{m}_{\mathrm{H}} \gtrsim 180 \mathrm{GeV}$. Different production modes, and the subsequent decays of the $\mathrm{W}$ and $\mathrm{Z}$ bosons, determine the signatures that can make a high mass search more or less powerful.

For each one of the $\mathrm{W}$ from $H \rightarrow W^{+} W^{-}$, the branching ratio for the leptonic decay is $\mathrm{BR}(\mathrm{W} \rightarrow \ell \nu, \ell=e, \mu, \tau)=32 \%$. Unfortunately, tau leptons cannot be efficiently identified, restricting the final states that can be easily reconstructed to those ones containing electrons and muons. The probability of both Ws decaying to an identifiable lepton $\left(\mathrm{e}^{+} \mathrm{e}^{-}, \mu^{+} \mu^{-}\right.$, or $\mathrm{e}^{ \pm} \mu^{\mp}$ ) is therefore $4 \%$. Despite its relatively low probability, the fully leptonic decay $\mathrm{H} \rightarrow \mathrm{W}^{+} \mathrm{W}^{-} \rightarrow \bar{\ell} \nu \ell \bar{\nu}$ is the one providing the channel with the best signal-to-background ratio. The strength of this search channel resides in its clear experimental signature: two oppositelycharged leptons accompanied by missing energy, which the gg $\rightarrow \mathrm{H} \rightarrow \mathrm{W}^{+} \mathrm{W}^{-} \rightarrow \bar{\ell} \nu \ell \bar{\nu}$ process exhibits. The only other SM process with the same signature is the non-resonant WW production; since $\sigma(\mathrm{p} \overline{\mathrm{p}} \rightarrow \mathrm{WW})=11.7 \mathrm{pb}$, the number of events due to this background is comparable to the potential number of signal events. In addition, at leading order, no jets are expected for both $H \rightarrow W^{+} W^{-}$and WW. This allows for the rejection of events due to larger backgrounds, such as $\mathrm{W}(+$ jets $/ \gamma)$ and $\mathrm{Z} / \gamma^{*}(+$ jets $/ \gamma)$, which are typical at a hadron collider. Some jet activity, however, is predicted by higher order diagrams, in which one or more gluons can be radiated by the incoming quarks (for WW) or gluons (for gg $\rightarrow H$ ). In addition to gg $\rightarrow \mathrm{H}$, secondary signal contributions arise from the associated production and vector boson fusion processes. Their cross section, however, is almost ten times smaller, and their final states are less clean, with additional leptons or jets from the scattering quarks or 
from the weak boson decays.

The same-sign dilepton final state is one exceptional case in which the associated production plays an important role within the high-mass searches. When the Higgs boson is produced in association with a weak boson, and they both decay leptonically, there is a non-negligible chance that one of the leptons is not reconstructed, for example due to the detector acceptance. In that case, it is possible to search for two leptons with the same sign, a signature that can rarely be produced by SM processes.

Beside the fully-leptonic decay of the Higgs boson, the semi-leptonic channel $\mathrm{H} \rightarrow$ $\mathrm{W}^{+} \mathrm{W}^{-} \rightarrow \bar{\ell} \nu j j$ is viable as well. However, its sensitivity is limited due to the larger backgrounds with jets, and to the limited resolution in the dijet mass.

For mass values close to or above $\mathrm{m}_{\mathrm{H}}=190 \mathrm{GeV}$, the $H \rightarrow \mathrm{ZZ}$ decay provides some sensitivity. The SM production of non-resonant ZZ pairs is the only intrinsic background, and selections based on the invariant mass of the dilepton or dijet provide a good way to reject background events. This search is pursued mostly at the LHC, where a better signal-to-background ratio and higher energy make this one of the best channels for a heavy Higgs boson. 


\section{CHAPTER 2}

\section{HADRON COLLIDER PHYSICS AT THE TEVATRON}

One way to verify the predictions of the standard model is to study high-energy collisions between fundamental particles. In this high-energy regime, the predictions of the SM are calculable, and can be tested with precise measurements. In addition, events in which large momenta are exchanged also allow for the production of massive particles, such as the Higgs boson.

The data used in this search were collected by the DØ detector at the Fermilab Tevatron collider. This chapter contains a description of the Tevatron, and an overview of a typical hadron collider experiment.

\subsection{Coordinates and Units}

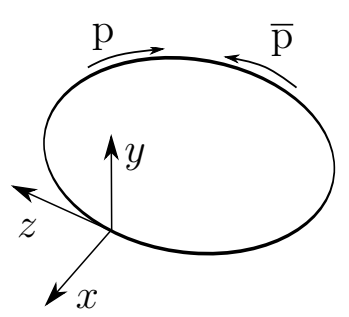

In the work described in this thesis, a right-handed coordinate system is used, with the positive $x$-axis pointing outside the collider ring, and the positive $y$-axis pointing up. Cartesian $(x, y, z)$, cylindrical $(r, \phi, z)$ and spherical $(r, \phi, \theta)$ coordinates are used throughout this work. When describing high- $p_{\mathrm{T}}$ physics, however, it is often convenient to use the pseudorapidity $\eta$ instead of the polar angle $\theta$. The pseudorapidity is defined in equation (2.1): 


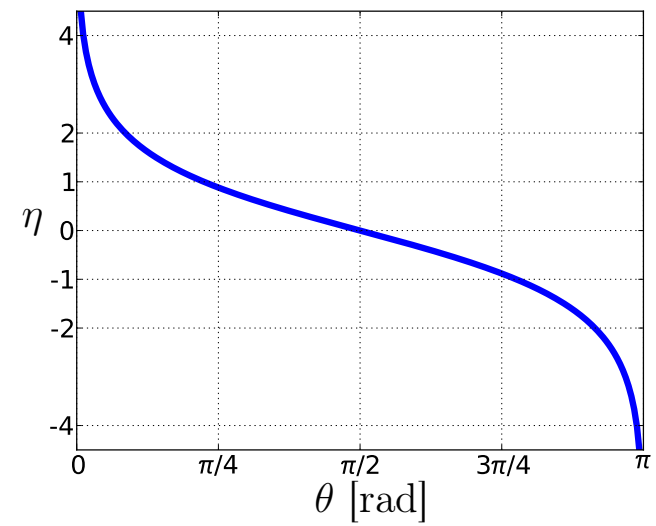

$$
\eta=-\ln \left(\tan \frac{\theta}{2}\right)
$$

The pseudorapidity approximates the rapidity $y$

$$
y=\frac{1}{2} \ln \left(\frac{E+p_{z}}{E-p_{z}}\right)
$$

in the massless limit $m \ll E$, where $m$ is the invariant mass $m=\sqrt{E^{2}-p^{2}}$. Instead of momentum ${ }^{1}$ and energy, it is also often convenient to use their transverse components: the transverse momentum $p_{\mathrm{T}}=p \sin \theta$, and the transverse energy $E_{\mathrm{T}}=E \sin \theta$. These two quantities are particularly useful at a hadron collider, where the colliding objects are composite particles made of partons (quarks and gluons). Each one of the two interacting partons carries a fraction $\left(x_{1}\right.$ and $\left.x_{2}\right)$ of the total hadron momentum. While the total longitudinal momentum might be non-zero (when $x_{1} \neq x_{2}$ ), the transverse momenta are small, and usually negligible compared to the center of mass energy.

The rate $R$ of a scattering process is given by

$$
R\left[\mathrm{~s}^{-1}\right]=\mathcal{L}\left[\mathrm{cm}^{-2} \mathrm{~s}^{-1}\right] \sigma\left[\mathrm{cm}^{2}\right]
$$

where the cross section $\sigma$ is the interaction probability per unit flux, and the instantaneous luminosity $\mathcal{L}$ is the particle flux. Cross sections are usually expressed in barns or one of its submultiples, where $1 \mathrm{~b}=10^{-24} \mathrm{~cm}^{2}$. During the ongoing Tevatron RunII, $\mathcal{L}$ is typically in

\footnotetext{
${ }^{1}$ Throughout this thesis I will use units such that $c=1$. With this choice, energy, momentum, and mass are all measured in $\mathrm{GeV}$
} 
the range of $0.6-3.8 \cdot 10^{32} \mathrm{~cm}^{-2} \mathrm{~s}^{-1}$. The number of events accumulated for a given process having cross section $\sigma$ is given by integrating in time:

$$
N=\sigma \int \mathcal{L} d t=\sigma \mathrm{L}
$$

where $\mathrm{L}=\int \mathcal{L} d t$ is the integrated luminosity. During the Tevatron RunII, which started in 2002, and which is scheduled to end in September 2011, $12 \mathrm{fb}^{-1}$ of data should be accumulated. For example, the process $\mathrm{p} \overline{\mathrm{p}} \rightarrow \mathrm{Z} \rightarrow \mu \mu$ has $\sigma=\sigma_{\mathrm{Z}} \times B R(\mathrm{Z} \rightarrow \mu \mu)=250 \mathrm{pb}$, so this dataset contains more than a million $\mathrm{Z} \rightarrow \mu \mu$ events .

\subsection{Proton-Antiproton Collisions at the Tevatron}

The Tevatron is a 1-km radius superconducting accelerator ring at Fermilab. It was the first synchrotron to use superconducting magnets; these Niobium-Titanium dipoles and quandrupoles can produce fields up to $4.2 \mathrm{~T}$, which are necessary to keep 980-GeV protons and antiprotons on their circular orbits.

The proton and antiproton beams are prepared in the Fermilab accelerator complex, shown in figure 2.1. Before reaching $980 \mathrm{GeV}$, they are accelerated and steered through a chain of magnets, radio-frequency (RF) cavities, and targets. Once injected into the Tevatron, these two counter-rotating beams are brought into collision at two points of the ring. Located at these two points are the $\mathrm{CDF}$ and the $\mathrm{D} \varnothing$ detectors, which record the outcome of these collisions. This section describes the steps required to prepare the beams and to bring them into collision. For a more detailed review, see Ref. [21].

\subsubsection{The Accelerator Chain}

The proton beam is initiated by accelerating $\mathrm{H}^{-}$ions to $750 \mathrm{keV}$ with an electrostastic potential. A RF linear accelerator (LINAC) then increases the kinetic energy of the $\mathrm{H}^{-}$ions from $750 \mathrm{keV}$ to $400 \mathrm{MeV}$. The $\mathrm{H}^{-}$ions are then converted into protons by passing through 


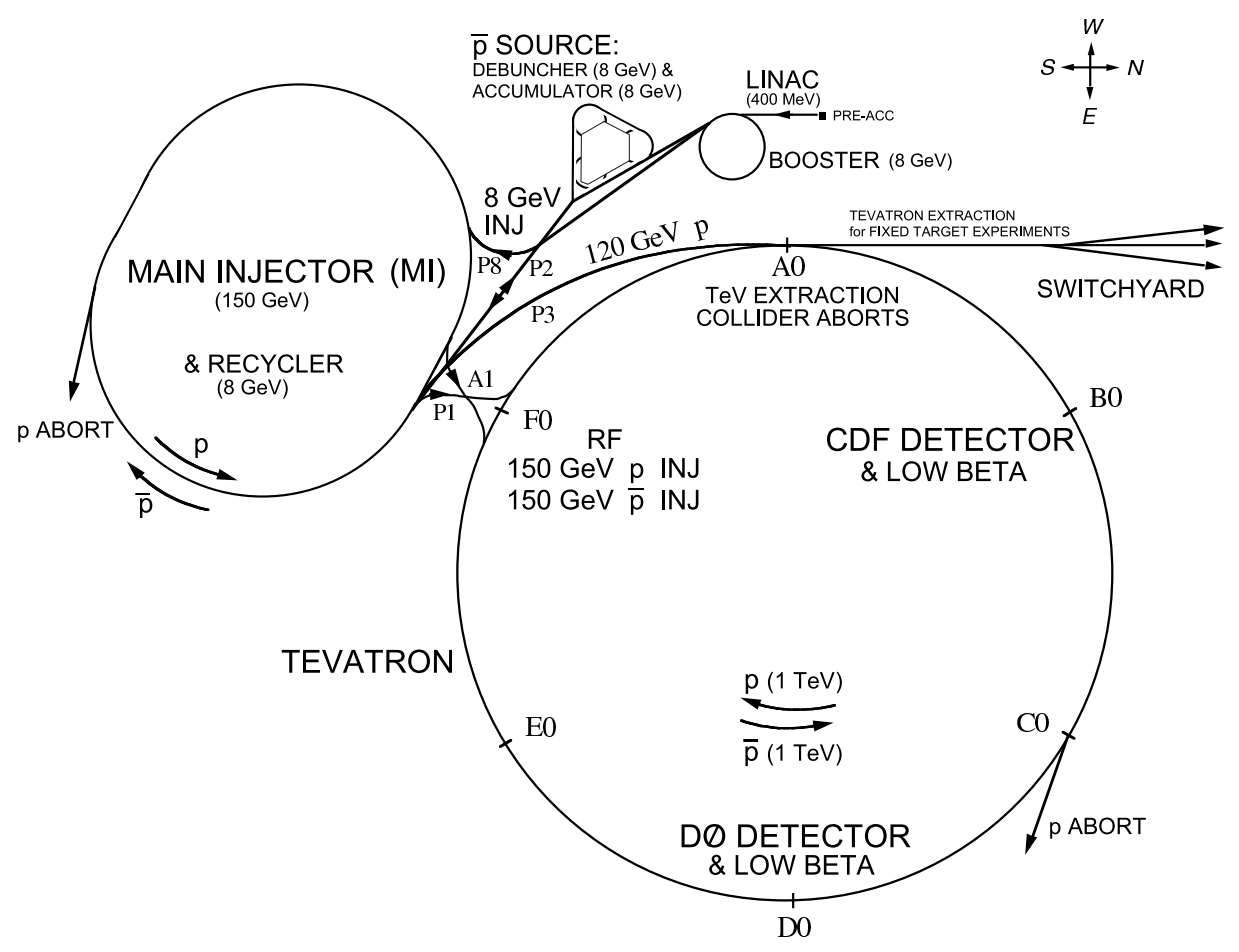

Figure 2.1: $\quad$ The Fermilab acceleration chain, from Ref. Add a brief description here with some of the details.

a carbon foil where the electrons are stripped off. From this stage onward, particles are accelerated with synchrotrons, machines where the circular trajectories of the beams are built around magnets (to steer and focus) and RF cavities (to provide the acceleration). Protons are then accumulated in the Booster, a synchrotron ring with conventional magnets. When about $10^{12}$ protons have been accumulated in the Booster, they are accelerated to $8 \mathrm{GeV}$, and at the same time grouped in bunches. Protons are then passed on to the Main Injector, a bigger synchrotron which can increase their energy up to $150 \mathrm{GeV}$. At this point the protons can either be injected to the Tevatron, or be used for the preparation of the anti-proton beam.

Anti-protons are generated by colliding the proton beam on a target. The shower of particles produced in this collision also includes some anti-protons, which are selected with a magnetic spectrometer. Millions of protons hitting the target are needed in order to produce tens of anti-protons. When enough anti-protons have been produced ${ }^{2}$ and stored in the

\footnotetext{
${ }^{2}$ Every 2.4 seconds about $5 \times 10^{12}$ protons are directed to the target. About $10^{11}$ antiprotons are produced every hour, and a stack of $1-2 \times 10^{12}$ antiprotons are required to initiate the collisions with a good instantaneous luminosity. This corresponds to slightly more than half a day, which is the typical duration of a collider store.
} 
Accumulator storage ring, the anti-proton beam is transferred to the Main Injector. There, they are accelerated to $150 \mathrm{GeV}$, and at that point they are ready to be transferred to the Tevatron.

\subsubsection{The Tevatron Main Ring}

After the injection of the 36 bunches of anti-protons, followed by the 36 bunches of protons, the two beams are circulating in opposite directions within the same beam pipe. They are then accelerated from $150 \mathrm{GeV}$ to $980 \mathrm{GeV}$, and squeezed to a diameter comparable to that of a human hair. They travel a few millimeters apart, their orbits spiraling around each other, except for two interaction points where they cross, and where the CDF and D $\varnothing$ detectors are located.

At these two locations a proton bunch goes through an anti-proton bunch every 396 ns. Most of the particles within each bunch continue traveling on their trajectories, but some of them collide head-on, with a center-of-mass energy of $1.96 \mathrm{TeV}$. The debris of these collisions, which take place at roughly the center of the CDF and DØ experiments, are detected and recorded by the two apparatuses. Collisions continue until the number of particles in each bunch is significantly reduced, and the beam quality has deteriorated. At that point, the proton beam is dumped, the remaining antiprotons are recycled, and new beams are injected. This cycle repeats about every 18 hours, and the time during which collisions take place is called a "store".

\subsection{High- $p_{\mathrm{T}}$ Physics}

High-energy experiments constitute large undertakings, not only because particles have to be accelerated to relativistic energies, but also because interesting scatterings are extremely infrequent. Millions of collisions per second and years of data collection are needed to observe such rare events. For this and for other reasons, the experimental apparatuses recording the 
collision outcome have to comply with several challenging specifications: they must provide high readout rates; they must be able to detect different types of particles, by employing several detector technologies; and they must cope with high radiation doses.

The collaborations that build these apparatuses generally involve hundreds (or even thoushands) of physicists and engineers from several institutions around the world. The specific implementation that each collaboration chooses for its detector can vary significantly. Nevertheless, the detector layout and the main experimental issues are the same across experiments, most of the time. This section describes a generic hadron collider detector, and the associated experimental issues.

\subsubsection{Typical Detector}

A typical detector in a collider experiment must be able to identify several particles that interact with matter in diverse and peculiar ways. Such a detector is composed of multiple subdetectors, each designed to measure one specific property of the particles, or to identify one particular type of them. A collider detector also needs to be hermetic, that is, to cover most of the solid angle around the collision point, so that it can capture most of the particles that are generated. The subdetectors are usually organized in concentric layers, surrounding the collision point with a cylindrical geometry. Subdetectors that moderately affect the particles are close to the center of the apparatus; the ones that can stop even the most penetrating particles are in the outer layers. The pieces of information gathered from each one of the subdetectors are combined, allowing for the identification of the particles, for the measurement of their trajectory, of their transverse momentum, and of their energy. What follows is a list of the subdetectors, in the order in which a particle would encounter them when traveling from the collision region outward.

Tracking The tracker measures the trajectory of charged particles. Their track is reconstructed from the energy depositions that they leave, by means of ionization, along their path. 
The position of the particle along its trajectory is measured at several points, by the detector layers that are located at different radii. In order to minimize the trajectory perturbations due to the interactions of the particle going through the detector, trackers are usually built with a small material budget. Thus, they often employ the following detector technologies: semiconductors, gas chambers, and plastic scintillators.

Frequently, trackers are immersed in a uniform magnetic field; this causes a bending of the trajectory, allowing for the measurement of the tranverse momentum $p_{\mathrm{T}}$. In addition, if two or more tracks originate from the same region, they are also used to determine the coordinates of their vertex. The primary vertex, that is the location where the scattering took place, is close to the beamspot. Secondary vertices, where short-lived particles decay, are displaced by a visible distance (hundreds of $\mu \mathrm{m}$ ), which depends on the lifetime of the unstable particle.

Calorimetry The calorimeter measures the energy of the particles that are subject to electromagnetic or hadronic interactions, by absorbing all their energy and stopping them in the detector. The energy is deposited in the calorimeter material by developing a shower of particles, and is released in the volume within which the shower evolved.

A shower initiated by a particle that interacts electromagnetically (a phothon or an electron) is smaller than the one initiated by a particle that interacts hadronically (mesons and barions). For this reason, the calorimeter is generally split in (at least) two compartments: the inner electromagnetic one, which is finely segmented, and the outer hadronic one, which is coarsely segmented. For the same reason, hadronic calorimeters are often organized in alternating layers: active ones, in which the energy deposited by the shower produces a detectable electronic signal; and passive ones, in which the deposited energy is not detected. Calorimeters in which only a fraction of the energy is measured are called sampling calorimeters; the sampling technique is used to reduce the calorimeter size, by building the absorber layers out of dense materials. 
Another important consequence, besides the different shower size, arises from the distinct phenomena that underlie electromagnetic and hadronic showers: in general, the electromagnetic response is higher than the hadronic one. It is possible, although not easy, to make the two responses about the same. This is accomplished by building the active and passive layers in the right proportions and with the appropriate materials. In that case, the calorimeter is said to be compensating.

The Muon System Muons are the only long-lived ionizing particles that can pass through the tracker and the calorimeter. Hence, muon detectors constitute the outermost layer, outside of the calorimeter. In general, they use two detection tecnologies: a fast one (scintillators or gaseous avalanche detectors), providing trigger information; and a slower one (for example gaseous drift tubes), providing more precise spatial information. If there is an outer magnetic field, the track stub reconstructed with the muon detectors allows for a measurement of the muon $p_{\mathrm{T}}$, in addition to the one from the tracker.

\subsubsection{Typical Problems at a Hadron Collider Experiment}

Several experimental issues are unique to the analyses of colliding-beam hadron experiments. Here, I outline the most relevant ones for this thesis. For a more detailed discussion see the review articles [22-24].

Particle identification When we say that we identify a particle in a hadron collider event, we mean, in fact, that we observe features corresponding to the ones that we expect for that type of particle. One should always keep in mind that particle identification is an intrinsically probabilistic process. For example, what we (experimentally) call a muon, is just a reconstructed track which happens to be in correspondence of a signal in the outer muon detector, and it thus looks like a muon. Similarly, a photon is a narrow energy deposit in the calorimeter that is consistent with an electromagnetic shower; an electron is a photon which also has a track pointing to it; light quarks $(\mathrm{u}, \mathrm{d}, \mathrm{s})$ and gluons are identified as jets of 
particles reconstructed as almost-collinear tracks and showers; neutrinos can only be inferred from an imbalance in the total transverse energy.

The identification is more involved for particles whose lifetime is less than few a nanoseconds, that is, short enough to allow their decay within the detector volume. A tau is identified by its decay products (mostly charged hadrons and neutral pions). Charm and bottom quarks are reconstructed as jets whose tracks belong to a displaced vertex. A W boson is identified by the decay products from its leptonic $(\mathrm{W} \rightarrow \ell \nu)$ and hadronic $(\mathrm{W} \rightarrow j j)$ decays. It should be noted, however, that the $\mathrm{W}$ mass cannot be determined with precision due to the presence of a neutrino or jets, and therefore cannot be used to select Ws; this is further discussed in section 5.4.1. As for the $\mathrm{W}$, the $\mathrm{Z}$ boson is identified by the decay products from its leptonic $(\mathrm{Z} \rightarrow \ell \ell)$ and hadronic $(\mathrm{Z} \rightarrow j j)$ decays. While $\mathrm{Z} \rightarrow j j$ events are difficult to select (due to the much larger dijet background), $\mathrm{Z} \rightarrow \ell \ell$ ones are not, and they constitute a reference subset that proves helpful for normalization and calibration. Top quarks, most of the times, are produced in t $\bar{t}$ pairs and decay via $\mathrm{t} \rightarrow \mathrm{Wb}$; they are identified by the decay products of $\mathrm{t} \overline{\mathrm{t}} \rightarrow \ell \nu \mathrm{b} \overline{\mathrm{b}} j j$ and $\mathrm{t} \overline{\mathrm{t}} \rightarrow \ell \nu \ell \nu \mathrm{b} \overline{\mathrm{b}}$.

This is a simplified list of features that are used to identify particles; nevertheless, it should suffice to show that there is a non-negligible chance of incorrectly associating two of these features, and of making a mistake in the particles' identification. This is particularly true in a high-multiplicity environment, such as the one at a hadron collider experiment.

Trigger The $\mathrm{p} \overline{\mathrm{p}}$ collision rate is much higher than the rate at which events can be read out and recorded. At the Tevatron, the $1.7 \mathrm{MHz}$ average collision rate is determined by the $6.28 \mathrm{~km}$ circumference and by the beam structure, with 36 bunches traveling close to the speed of light. Even just transmitting the data ouside the collision hall would be impossible at this rate. Furthermore, each event corresponds to about 250 kilobytes of data. Storing all the events would require a sizeable capacity, of the order of few terabytes per second. Finally, most of the collisions are elastic ones, which are not an interesting subject of study. Thus, 
only a very small fraction of events (typically one in a hundred of thousands) is recorded for subsequent offline analysis. Deciding, within microseconds, whether or not an event can be discarded is one of the major challenges at a hadron collider experiment. The trigger system is a set of hardware and software components rejecting the events that are not likely to be of any interest.

Energy and momentum calibration At a hadron collider, the momentum calibration and the energy calibration constitute difficult tasks; this is because the center of mass energy is not constrained by the beam energy. Calibration data is gathered before the detector installation, with test-beam studies, and afterwards, during everyday data taking. The momentum scale for track measurements is determined from known resonances, such as the $\mathrm{J} / \psi$ and $\mathrm{Z}$, that decay to muon pairs. Similarly, known resonances decaying to electron or phothon pairs can be used to determine the energy scale for electromagnetic showers. The calibration of the calorimeter response to jets is more challenging; in order to use a known dijet resonance, one would need to implement a trigger selecting such resonance from the large multijet background. Rather than pursuing this arduous approach, the jet energy scale is often determined using $\gamma+$ jet events, by balancing the transverse momentum of the jet recoiling against the photon.

Beamspot length and multiple interactions. The bunches of protons and antiprotons have a fusiform shape, a few micrometers wide and almost one meter long. As a consequence, the $\mathrm{p} \overline{\mathrm{p}}$ collisions take place in a region, the beamspot, that is precisely defined in the $x-y$ plane, but not in the beam direction: the $z$-coordinate of the interaction point is distributed with an RMS of $30 \mathrm{~cm}$. Although tracking detectors can resolve spatial coordinates with micrometric precision, the spread of the vertex position along the beamline has two major consequences.

First, one complication arises when calculating transverse energies. Because they are computed multiplying the energies measured in the calorimeter by $\sin (\theta)$, the polar angle 
$\theta$ cannot be the one determined with the coordinate origin at the center of the detector. Instead, $\theta$ is the angle between the beam direction and the line running from the vertex to the location of the calorimeter cells that measured the energy. This complication is not there for the $p_{\mathrm{T}}$ measured with the tracker, where the track curvature provides direct information about the momentum's transverse component.

Second, interactions that take place in the same bunch crossing can be disentangled, for the most part. There are two reasons for which one single bunch crossing can produce more than one interaction: either because multiple partons interact within one single $p \bar{p}$ collision, or because more than one $\mathrm{p} \overline{\mathrm{p}}$ pairs collide. Events due to the former are called "multiple interactions", and those due to the latter are called "pile up". Most of the time, these interactions produce jets, which can complicate the reconstruction of the hard-scattering event. While multiple-interaction jets originate from the same position of the hard scattering, pile-up jets usually originate from a different position, due to the longitudinal spread of proton and antiprotons within a bunch. Pile-up jets can therefore be identified as such, provided that there are enough tracks within the jet cone to determine the location of their vertex. Separating jets from different locations allows for a more accurate estimate of the number of jets from the hard scattering ${ }^{3}$.

Normalization The number of events expected for each process is normalized to the product of the cross section for that process and the total integrated luminosity. In turn, the integrated luminosity is usually normalized to the total $\mathrm{p} \overline{\mathrm{p}}$ cross section, $\sigma_{\text {tot }}$. A calculation from first principles of $\sigma_{\text {tot }}$, however, would require a precise knowledge of the beam sizes and fluxes, which we don't have. Another approach, used in the work described here, is to use the total integrated luminosity as a starting point, and then normalize all cross-sections to that of $\mathrm{Z} / \gamma^{*}(+$ jets $/ \gamma)$. This cross-section suits well as a reference: it can be computed easily, it is directly related to the Drell-Yan process used to fit the global parton distribution

\footnotetext{
${ }^{3}$ The next logical step, after counting the jets that originate from the hard scattering, would be to neglect the other jets when one computes the missing transverse energy. This idea has not been implemented yet, because it is difficult to disentangle energies in the calorimeter that migh be due to partially overlapping jets.
} 
functions, and can be measured precisely thanks to its distinctive signature. A description of the normalization scheme used in this analysis can be found in section 5.1. 


\section{CHAPTER 3

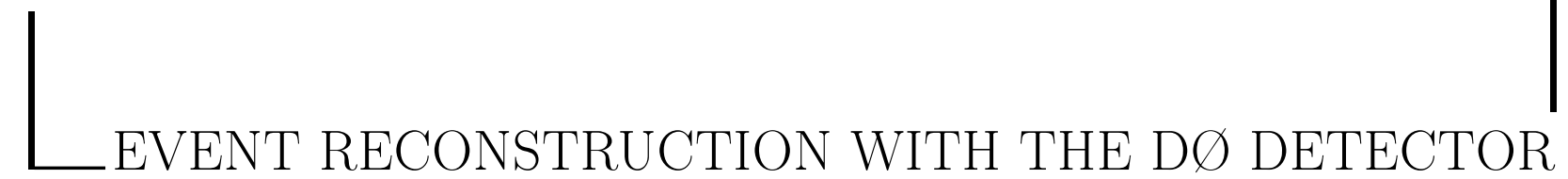

The DØ experiment was proposed in 1984 by a worldwide collaboration of scientists. The eponymous DØ detector was built in the following years, and it started collecting data at the Tevatron collider in 1987. The detector is extensively described elsewhere [25; 26], and here I review only those aspects that are relevant to the present investigation.

The $\mathrm{D} \varnothing$ detector functions as an advanced camera: in the same way that photons produce a set of electronic signals that can then be reconstructed as an image, the collision fragments go through the different parts of the detector, and produce a set of signals that can then be combined to provide an overall picture of the collision. The reconstruction of each collision, or event, is performed in three main steps. First, the bits from within each one of several subdetectors are combined: hits from the tracker are joined and fit into tracks, energy deposits from the calorimeter are combined into showers and jets, and hits from the muon system are used to find muon tracks. An overview of the subdetectors composing the $D \varnothing$ detector is shown in figure 3.1. Second, the pieces of information from different subdetectors are

combined. This allows for the identification of the particles, and for the measurement of global quantities, such as the missing transverse energy. In the third step, corrections that are specific to each object are applied to the quantities that have been measured. 


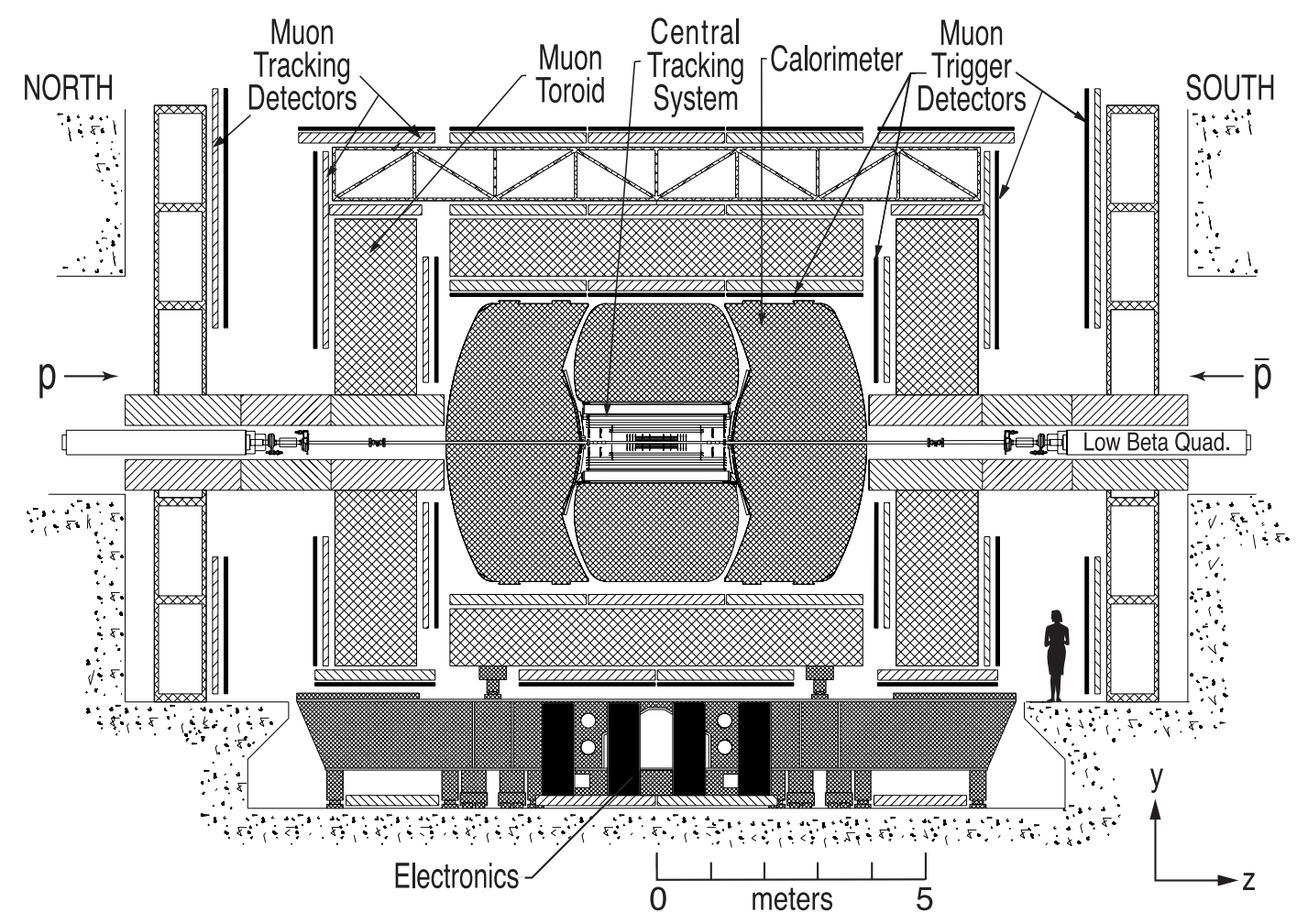

Figure 3.1: Overview of the D $\varnothing$ detector, from Ref.[26]

In the following sections, I describe the different subdetectors that compose the $\mathrm{D} \varnothing$ detector, and the pieces of information that each one of them provides. I will then describe the procedures used to reconstruct an event, that is to combine those pieces of information and identify physics objects such as leptons, jets, and missing energy.

\subsection{Tracking}

When a charged particle goes through material, it interacts with the atoms in the material, mainly by leaving energy deposits along its trail. Although these energy losses might be negligible in comparison to the energy of a relativistic particle, thus causing only a marginal deceleration, they can deflect its path and cause multiple scatterings. The multiple scattering effect is characterized by the formula (3.1), describing the angular dispersion $\sigma_{\theta}$ as a function 
of the material budget in units of radiation legths $X_{0}$ :

$$
\sigma_{\theta}=\frac{13.6 \mathrm{MeV}}{\beta p \mathrm{c}} \sqrt{\frac{X}{X_{0}}} .
$$

Tracking detectors, which are meant to measure the particles' paths, are built with materials and geometries designed to minimize these distorsions, and to maximize the precision with which the trajectory can be measured. For this reason, most of the time they are built as concentric layers of thin detectors, each providing one spatial measurement.

Trackers are usually immersed in a strong magnetic field, which allows for the simultaneous measurement of the trajectory and, from the track curvature, of the transverse momentum $p_{\mathrm{T}}$. The transverse momentum resolution is determined by the precision with which the track sagitta $s$ can be measured; such precision is limited by the spatial resolution of each layer and by the number of layers. To a first order approximation, the transverse momentum is inversely proportional to the sagitta, as indicated in equation (3.2).

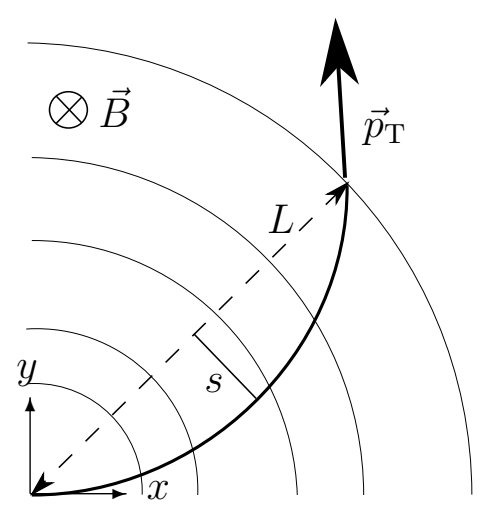

$$
s \simeq \frac{0.3 \mathrm{~B}[\mathrm{~T}] \mathrm{L}^{2}[\mathrm{~m}]}{8 p_{\mathrm{T}}[\mathrm{GeV}]}
$$

For example, the $\mathrm{D} \emptyset$ tracking system is housed within a $2 \mathrm{~T}$ superconducting solenoidal magnet, which has a length of $2.8 \mathrm{~m}$ and an inner radius of $60 \mathrm{~cm}$. With these parameters, the track of a muon with a transverse momentum of $10 \mathrm{GeV}$ has a sagitta of $2.7 \mathrm{~mm}$. This requires the tracking detector to have micrometric precision, and at the same time to be built with components that have a very low ${ }^{1}$ material budget. Furthermore, given the high flux of ionizing particles in the region around the interaction point, these components must be

\footnotetext{
${ }^{1}$ The material budget of the $\mathrm{D} \varnothing$ tracking system amounts to less than one radiation length [27].
} 


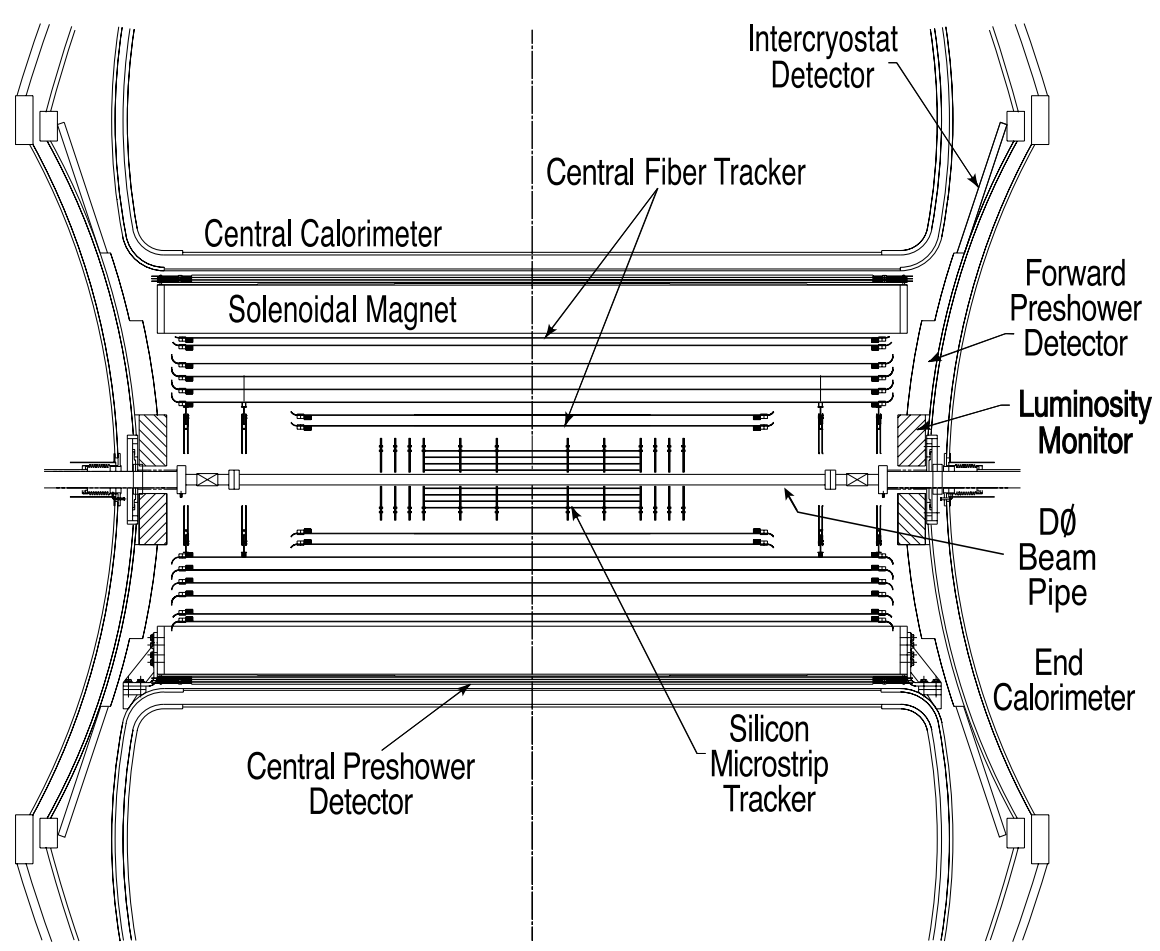

Figure 3.2: $\quad$ Crosssectional view of the $\mathrm{D} \varnothing$ RunII tracking system, from Ref. [26]. The SMT and the CFT are contained within the solenoid. The CFT layers are organized in concentric cylinders, while the SMT layers are either cylinders or disks. Outside the solenoid are the preshower detectors and the luminosity monitor.

resistant to radiation damage.

After recording several hits for one particle, each hit corresponding to a spatial measurement in one layer, the hits are combined to reconstruct the trajectory of the particle. Tracks that originate from a common point in space are then combined to reconstruct their vertex of origin.

\subsubsection{Layout of the Tracking Detectors}

The D $\varnothing$ tracking system is composed of several layers; the number of layers traversed by a particle originating from the interaction point, at the center of the detector, varies as a function of $\eta$ between 6 and 24. The inner layers of the tracking system, where tracks have a higher density and a finer granularity is needed, are made of silicon microstrip detectors. The outer layers, where a coarser granularity is sufficient, are made of scintillating fibers. The layout of the tracking detectors is show in figure 3.2. 
The Silicon Microstrip Tracker (SMT) The SMT is composed of more than 600 basic detectors, also called modules, arranged in 5 concentric cylindrical layers and 16 disks, as described in Ref. [28]. Each module is made of a 300- $\mu$ m thick silicon wafer along with its front-end electronics; the silicon is segmented in parallel strips with pitch varying from $50 \mu \mathrm{m}$ to $150 \mu \mathrm{m}$. An inverse bias is applied to deplete the doped silicon, and to collect electron-hole charges created by the passage of an ionizing particle.

The arrangement of the SMT modules in disks and barrels is dictated by the elongated shape of the interaction region, described in section 2.3.2. This layout, with interspersed barrels and disks, guarantees that high- $p_{\mathrm{T}}$ tracks, despite originating from a $z$-position with a 30-cm RMS, are perpendicular to the detector surfaces even at high $\eta$. The advantage of such a choice is that the spatial resolution is better for tracks that are perpendicular to the silicon plane in which the strips lie.

The SMT tracker was first built with six barrels and 16 disks. Subsequently, the SMT was upgraded with the introduction of one additional layer of modules on a cylinder around the beampipe; the main goal of this upgrade, described in Ref. [29], was to compensate for the aging of the inner layers due to radiation damage. Each barrel has four cylindical layers, with radii from $2.7 \mathrm{~cm}$ to $10.5 \mathrm{~cm}$. The disks are of two types: $12 \mathrm{~F}$-disks with $9.96 \mathrm{~cm}$ outer radius, and $4 \mathrm{H}$-disks with $25 \mathrm{~cm}$ outer radius. Four F-disks are located between the barrels, while the remaining disks are ouside the barrels, in the high- $\eta$ region.

The Central Fiber Tracker (CFT) The CFT is made of scintillating fibers with a $835 \mu \mathrm{m}$ diameter, mounted on 16 concentric cylinders. The radius of the cylinders varies from $20 \mathrm{~cm}$ to $51.6 \mathrm{~cm}$; fibers within each cylinder are either parallel to the beam direction, for axial layers, or tilted with a stereo angle of $\pm 3^{\circ}$, for stereo layers. Going from the innermost cylinder outward, the axial and stereo layers alternate. The length of the 12 outermost cylinders is $2.52 \mathrm{~m}$, while the innermost 4 are shorter, $1.66 \mathrm{~m}$, in order to accomodate for the SMT H-disks. 
The following two subdetectors, the preshower and the luminosity monitor, are not strictly part of the tracking system; nevertheless, they are located within the extended tracking volume, so I describe them at this point.

Preshower Detectors The Central Preshower (CPS) and the Forward Preshower (FPS) are two subdetectors located on the inner faces of the calorimeter cryostats. They are composed by layers of scintillating fibers, three in the CPS and four in the FPS, and a layer of lead absorber. They provide additional tracking information, which is mostly used for the identification of EM objects.

Luminosity Monitor Located between the H-disks and the end calorimeters is the luminosity monitor. This system, composed of 24 plastic scintillator wegdes covering $2.7<|\eta|<4.4$, is used to detect the remnants from inelastic collisions. The luminosity monitor measures the instantaneous luminosity by counting the number of empty bunch crossings.

\subsubsection{Track Reconstruction}

The reconstruction of a track is performed in three main steps. First, the hits from adjacent electronics channels, within the same silicon module or fiber layer, are grouped into clusters. Second, track hypoteses are constructed by associating clusters that may belong to the same trajectory. Third, the parameters of the hypothesized tracks are accurately determined with a fit.

Each track is typically reconstructed from $10-20$ hits, and more than 100 tracks can be generated in a single event with high instantaneous luminosity. Given the high number of possible combinations that one can construct from the large number clusters of a single event, the most challenging issue with the track reconstruction procedure is the identification of real tracks. This step is called track finding, and at DØ two algorithms [30; 31], with complementary limitations and strenghts, are used to reconstruct tracks.

The $p_{\mathrm{T}}$ resolution of the $\mathrm{D} \varnothing$ tracker is shown in figure 3.3. It is usually parametrized as 


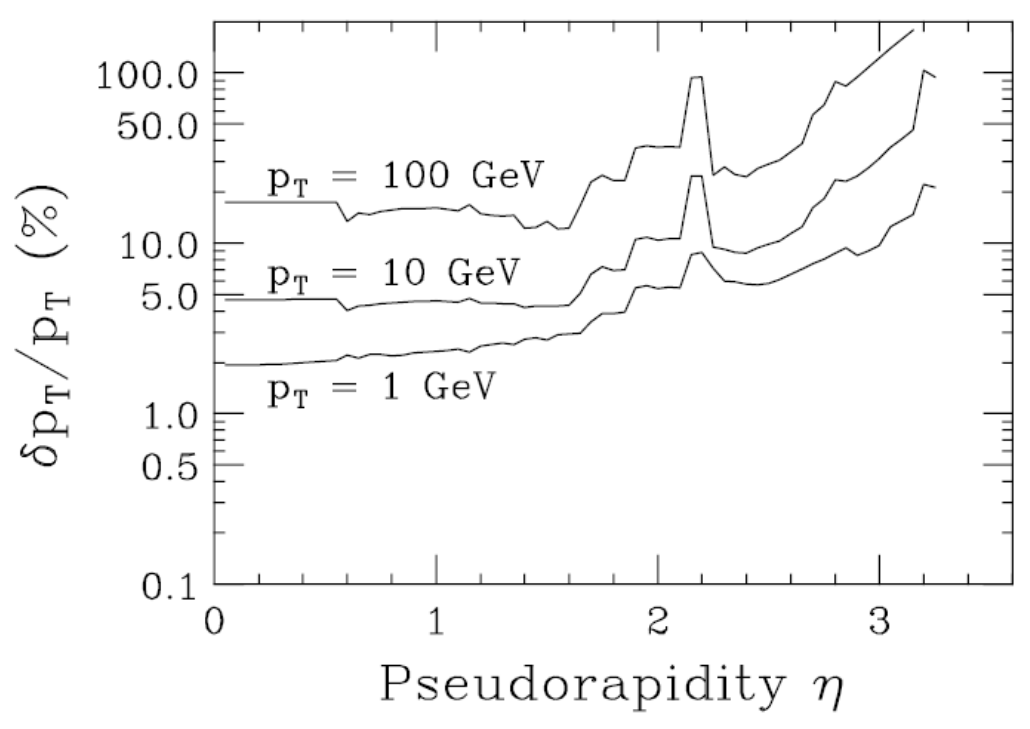

$$
\frac{\sigma_{1 / p_{\mathrm{T}}}}{1 / p_{\mathrm{T}}}=\sqrt{A^{2} \frac{p_{\mathrm{T}}^{2}}{L^{4}}+\frac{B^{2}}{L \sin \theta}}
$$

where the first term corresponds to the single hit resolution, and the second term to the multiple scattering. The tracker momentum resolution is worse at higher energies, which correspond to smaller sagitta values. Conversely, calorimeter measurements become more precise with increasing energy, as described in section 3.2.

\subsubsection{Vertex Reconstruction}

A vertex is defined as the point in space from which multiple tracks originate. The reconstruction of vertices is done by grouping the tracks and performing a fit where they are constrained to a common origin. Starting from the set of all tracks that satisfy loose reconstruction requirements, the track that contributes the most to the $\chi^{2}$ of the fit is removed; the fit is then re-iterated until a pre-defined $\chi^{2}$ threshold is reached and at least two tracks are left. The same procedure is then repeated with the tracks that have not been assigned to any vertex, until there are tracks that can be matched to any other track.

Vertices can be due to high-energy collisions, in which case they are called primary vertices, or to particle decays, in which case they are called secondary vertices. 
Secondary vertices, for example, are reconstructed in the case of $B$-mesons, which can be produced within a jet initiated by $b$-quarks. These mesons travel several millimiters and then decay into lighter hadrons, whose tracks can then be associated with a secondary, displaced vertex. Algorithms based on the displacement of secondary vertices allow for the identification of jets initiated by $b$-quarks. The identification of $b$-jets constitutes a powerful data-analysis tool, usually referred to as $b$-tagging, which is further described in section 3.2.

As discussed in section 2.3.2, multiple collisions can occur in a single beam crossing. Thus, multiple primary vertices are usually reconstructed for one event. Only one of them, however, corresponds to the interesting collision with a high energy transfer. This event is usually referred to as the "hard scattering", while most of the other collisions within the crossing are "soft" ones, that is with low energy transfers. The hard scattering vertex is called the primary vertex, PV.

The identification of the primary vertex among the reconstructed vertices is performed by an algorithm, which is based on the number of high- $p_{\mathrm{T}}$ tracks associated with each one of them. In the course of this study, it was observed that this algorithm fails for a small percentage of the events. Misidentification of the PV can happen if two vertices with high- $p_{\mathrm{T}}$ tracks are found and, for example, the first vertex only has two tracks, while the second has several. In this case, the algorithm identifies the second one as the PV, while for a dilepton analysis the first one is the vertex of interest. We established a correction procedure, called re-vertexing, to recover these events and update the PV coordinates. This procedure also recomputes the energy transverse component for objects reconstructed with the calorimeter, such as jets, electrons, and $E_{\mathrm{T}}$, because the updated $z$-position of the vertex requires an updated $\sin \theta$ factor. 


\subsection{Calorimetry}

Calorimeters are blocks of material in which particles can interact and gradually deposit all of their energy until they are stopped. The energy deposition takes place either via electromagnetic interactions, pair production and bremsstrahlung, or hadronic interactions; in both cases, a multiplicative phenomenon takes place, in which a shower of secondary particles is generated.

The typical dimension of an electromagnetic shower is a radiation length, $X_{0}$. The typical dimension of a hadronic shower is an interaction length, $\lambda_{I}$. For all the materials practically used in the construction of calorimeters, a radiation length is shorter ${ }^{2}$ than an interaction length. Thus, hadronic showers are larger than electromagnetic ones, and their characteristic size is what determines the typical volume and granularity of calorimeters for a collider experiment. Because of the large volume required to contain a hadronic shower, hadronic calorimeters are often sampling ones, in which the deposited energy can be detected only in a fraction of the material, while the remaining part is passive absorber.

The basic processes involved in the two types of showers are different. Thus, in general, the calorimeter response to an electromagnetic shower is different from the response to a hadronic shower, in which case the calorimeter is said to be non-compensating. Compensation can be achieved with a careful choice of the active and passive materials, and of their relative proportions. Nevertheless, most of the calorimeters are non-compensating.

\subsubsection{Layout of the Calorimeter}

The $\mathrm{D} \varnothing$ calorimeter is a sampling calorimeter located outside the solenoid. Passive layers, made of depleted uranium and steel, alternate with liquid argon active layers, in which the ionization of argon generates a detectable electronic signal.

The central part of the calorimeter, CC, is located inside a central cryostat, and it covers

\footnotetext{
${ }^{2} X_{0}$ and $\lambda_{I}$ are parametrized as a function of the material properties by the formulas: $X_{0}=$ $\frac{716 \mathrm{~g} / \mathrm{cm}^{2} A}{Z(Z+1) \log (287 / \sqrt{Z})}, \lambda_{I} \sim 35 A^{1 / 3} \mathrm{~g} / \mathrm{cm}^{2}$. The numerical values for uranium are $X_{0}=0.32 \mathrm{~cm}$ and $\lambda_{I}=10.5 \mathrm{~cm}$.
} 


\begin{tabular}{lcc}
\hline Layer & CC & EC \\
\hline Outer CH & - & 6.0 \\
CH & 3.2 & 4.4 \\
\hline FH3 & 0.76 & 1.1 \\
FH2 & 1.0 & 1.1 \\
FH1 & 1.3 & 1.1 \\
\hline EM4 & 9.8 & 9.3 \\
EM3 & 6.8 & 7.9 \\
EM2 & 2.0 & 2.6 \\
EM1 & 1.4 & 1.6 \\
\hline
\end{tabular}

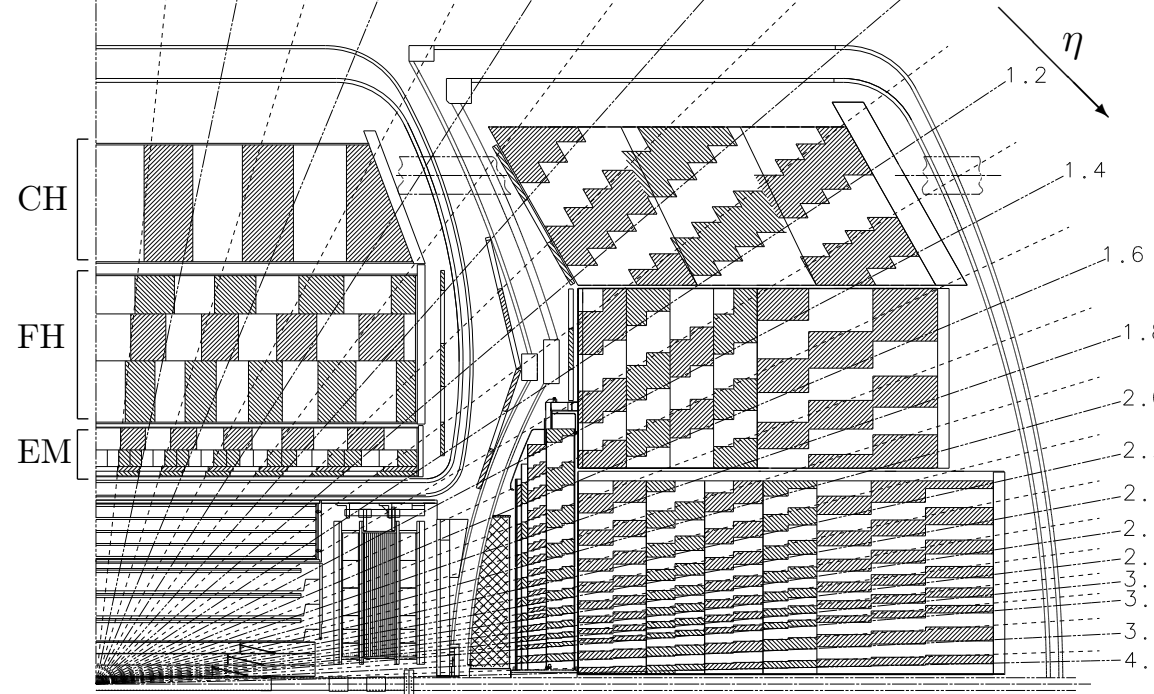

Figure 3.4: $y-z$ section of one quadrant of the D $\varnothing$ calorimeter, from Ref. [26]. Cells are arranged in projective towers, each tower of size $\Delta \eta \times \Delta \phi=0.1 \times 0.1$. Absorber thicknesses are indicated in the table. Electromagnetic layers are measured in radiation lengths $X_{0}$. Hadronic absorber layers are measured in interaction lengths $\lambda_{I}$.

the pseudorapidity region $|\eta|<1.2$. The north and south cryostats contain the two end parts of the calorimeter, EC, covering the region with pseudorapidity up to $|\eta| \simeq 4.5$. Cables and mechanical structures are located in the region between central and end cryostats. The energy deposited in this region is sampled with liquid argon cells, massless gap, and scintillating tiles, inter-cryostat detector, ICD.

The calorimeter readout is segmented in cells, shown in figure 3.4; This segmentation allows for the reconstruction of both shower position and shower longitudinal shape. The segmentation in the $(\eta, \phi)$ coordinates yields the tower structure; the segmentation in the radial direction yields to the layer structure. Most of the towers have dimensions of $0.1 \times 0.1$ in $(\eta, \phi)$ coordinates. The layers' thicknesses vary, as indicated in figure 3.4: the inner ones are a few radiation lengths thick, the outer ones are a few interaction lengths thick. The four innermost layers constitute the electromagnetic (EM) section, surrounded by three layers of the fine hadronic $(\mathrm{FH})$ section, and the outermost layer of the coarse hadronic $(\mathrm{CH})$ section. 


\subsubsection{Cluster and Jet Reconstruction}

Signals from neighboring calorimeter cells are grouped into clusters that correspond to showers. Different clustering algorithms are used for electromagnetic clusters and for hadronic ones.

Electromagnetic Clusters The clustering algorithm used to reconstruct electromagnetic objects starts from a list of seed EM towers. A tower corresponds to cells from different layers and located at the same $(\eta, \phi)$ coordinates. A seed EM tower must have a total transverse energy greater than $1.5 \mathrm{GeV}$, where the total transverse energy is obtained by summing the contributions from the EM layers and from the first hadronic layer. A calorimeter cluster is then built from the cells within a cone of radius $R=\sqrt{\Delta \eta^{2}+\Delta \phi^{2}}=0.4$. The cone is initially centered around the seed EM tower; in subsequent iterations, its center is computed again as the energy-weighted centroid, repeating the calculation until a stable cluster is found. Subsequently, if a matching cluster is found in the preshower, the EM object's quantities are updated: the energy from the preshower is included, and the position is adjusted accounting for this additional measurement. Energy corrections are then applied to electron and photons reconstructed with the EM cluster. The energy resolution of an EM object is shown in figure 3.5 .

When there is a reconstructed track pointing to the cluster center (within $\Delta \eta \times \Delta \phi=$ $0.05 \times 0.05)$, the EM cluster is considered to be an electron candidate. For electron candidates, additional quantities are computed to improve their correct identification and reject early showering hadrons. These quantities include, among others, the electromagnetic fraction, the isolation fraction, the spatial track match $\chi^{2}, E_{\mathrm{T}} / p_{\mathrm{T}}$, the H-matrix $\chi^{2}$, which provides information about the shower shape, and the electron likelihood, which is built from the probability distributions of the above variables for electrons and fakes.

Hadronic Clusters and Jets Quarks or gluons generated in a hard scattering produce jets. A jet is usually defined as a collection of collimated colorless hadrons, mostly pions and 


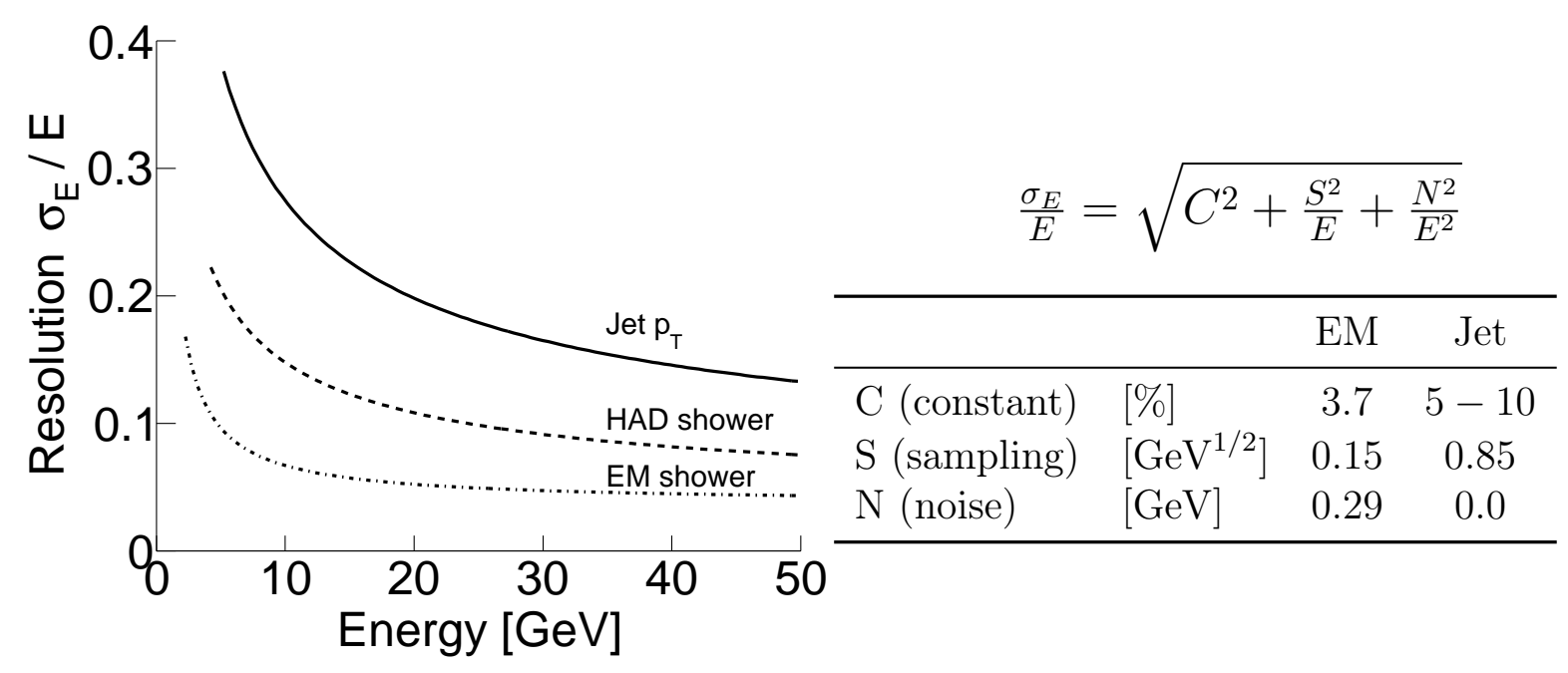

Figure 3.5: Typical energy resolutions for objects reconstructed with the D $\varnothing$ calorimeter. The energy resolution is parametrized with three terms: a constant term $C$ accounting for calibration and non-uniformities, a sampling term $S$ accounting for stochastic fluctuations, and a noise term $N$ accounting for fluctuations due to electronics, uranium decays, and pileup. The values of the parameters reported in the table are from Ref. [33; 34], and correspond to the resolution for objects reconstructed in the central calorimeter.

kaons, moving in a direction close to the trajectory of the original parton.

In practice, the definition of a jet depends on the algorithm used to reconstruct the jet, that is to convert the energy deposits due to neighboring hadrons showering in the calorimeter into a collection of clusters that we call jet. The algorithm used by most of $\mathrm{D} \varnothing$ analyses is the mid-point cone algorithm, described in Ref. [35].

First, towers with more than $0.5 \mathrm{GeV}$ are combined into proto-clusters. Then, the protoclusters that are within a cone of fixed radius ${ }^{3}$ are grouped together, and the center of the cone is iteratively determined as the $E_{\mathrm{T}}$-weighted centroid. The resulting list of proto-jets is enlarged by centering the cones on the mid-points of the corresponding pairs. The proto-jets are then updated with a split-and-merge procedure based on their overlapping towers and the corresponding energies. At this point, quality requirements are applied to remove those jets that could be due to EM clusters or to calorimeter noise.

The energy measured in a jet at the detector level does not usually match the one at particle level, that is the one of the original parton. There are a number of effects that can

\footnotetext{
${ }^{3}$ In this analysis the jets have been reconstructed with $R=0.5$.
} 
systematically bias the measurement, the most important of which are:

- non-linear calorimeter response, which can be due to non-compensation ${ }^{4}$ or noninstrumented regions;

- out-of-cone energy;

- energy offsets that are not due to particles from the hard scattering, for example electronic noise, pile-up, or minimum-bias interactions;

- muons produced by in-flight decays of heavy flavor hadrons;

The jet energy scale (JES) correction is applied to compensate for these effects. A second correction - based on a method called jet shifting, smearing, and removal (JSSR) - is applied to match the jet resolution observed in Monte Carlo with the one observed in data. A thorough description of the jet algorithms and corrections used at D $\varnothing$ can be found in Ref. [35].

Tracks inside a jet cone can provide important pieces of information regarding the jet. In the study described here we use two techniques relying on tracks within the jet: vertex confirmation and $b$-tagging.

Vertex confirmation is a technique to determine whether or not a jet originates from a given vertex. It requires that at least two tracks within the jet cone be associated with the event primary vertex. Because the resolution in the $z$ coordinate is much better for tracks than for jets, with this requirement we can improve the precision with which we count jets in an event. In particular, we can reject additional jets that are due to multiple collisions within one bunch crossing, thus originating from a different $z$ position.

A heavy-flavor likelihood, commonly referred to as $b$-tag output, can be associated to each jet. Before illustrating the $b$-tag procedure used at $\mathrm{D} \varnothing$, we shall define the jet taggability. In the same way that jets are built from calorimeter clusters, track jets are built grouping

\footnotetext{
${ }^{4}$ Many of the particles within a jet have a relatively low energy, as low as $2 \mathrm{GeV}$, and at this low energy the calorimeter response is often not linear.
} 
together neighboring tracks ${ }^{5}$. A calorimeter jet is "taggable" if its cone direction matches the one of a track-jet within a distance of $\Delta R<0.5$. A $b$-tagged jet is a taggable jet in which the tracks are associated to a displaced vertex. At D $\varnothing$, jets are not labeled with a binary value indicating whether or not the jet originated from a b quark; instead, a continous $b$-tag value is used. This value is computed combining several pieces of information. They include: the significance of the tracks displacements, the secondary vertex position, and the presence of muons ${ }^{6}$. These pieces of information are combined within a multivariate discriminant, whose continuous output is a measure of the $b$-tag likelihood. The efficiency and the fake rate of the $b$-tag algorithms vary significantly as a function of the jet $p_{\mathrm{T}}$ and $\eta$. Nevertheless, efficiencies above $50 \%$ with fake rates below $1 \%$ are obtained for energetic central jets. A detailed description of the $b$-tag techniques used at $\mathrm{D} \varnothing$, along with the resulting performance, can be found in Ref. [36].

\subsection{Muon System}

The muon identification at $\mathrm{D} \varnothing$ relies on the fact that muons are the only interacting particles that can reach the outer layers of the detector, the muon system, while all the other particles are stopped by the absorber material of the calorimeter. Therefore, any charged track that is reconstructed outside of the calorimeter volume is inferred to be due to a muon. The calorimeter outer radius is about $3 \mathrm{~m}$, hence the large surface covered by the muon system requires that its detectors have a coarse granularity and a limited resolution. The muon system is composed of two types of detectors: scintillators and proportional wire chambers. The scintillators provide fast and accurate timing information that is used to select events of interest. The wire chambers provide spatial information that allows for the reconstruction of the muon tracks. Furthermore, with the timing coincidence betweeen these two detectors,

\footnotetext{
${ }^{5}$ Quality requirements are applied on the tracks used to build a track-jet. These requirements are similar to the ones applied to the calorimeter clusters used to build standard jets. For example, they ensure that the tracks are reconstructed in a fiducial region of the detector, and that the tracks have a minimum $p_{\mathrm{T}}$.

${ }^{6} \mathrm{~A} b$-meson decays to a muon $10 \%$ of the time. Thus, the presence of a muon within a jet cone is an indication that the jet might be originating from heavy-flavor quarks.
} 


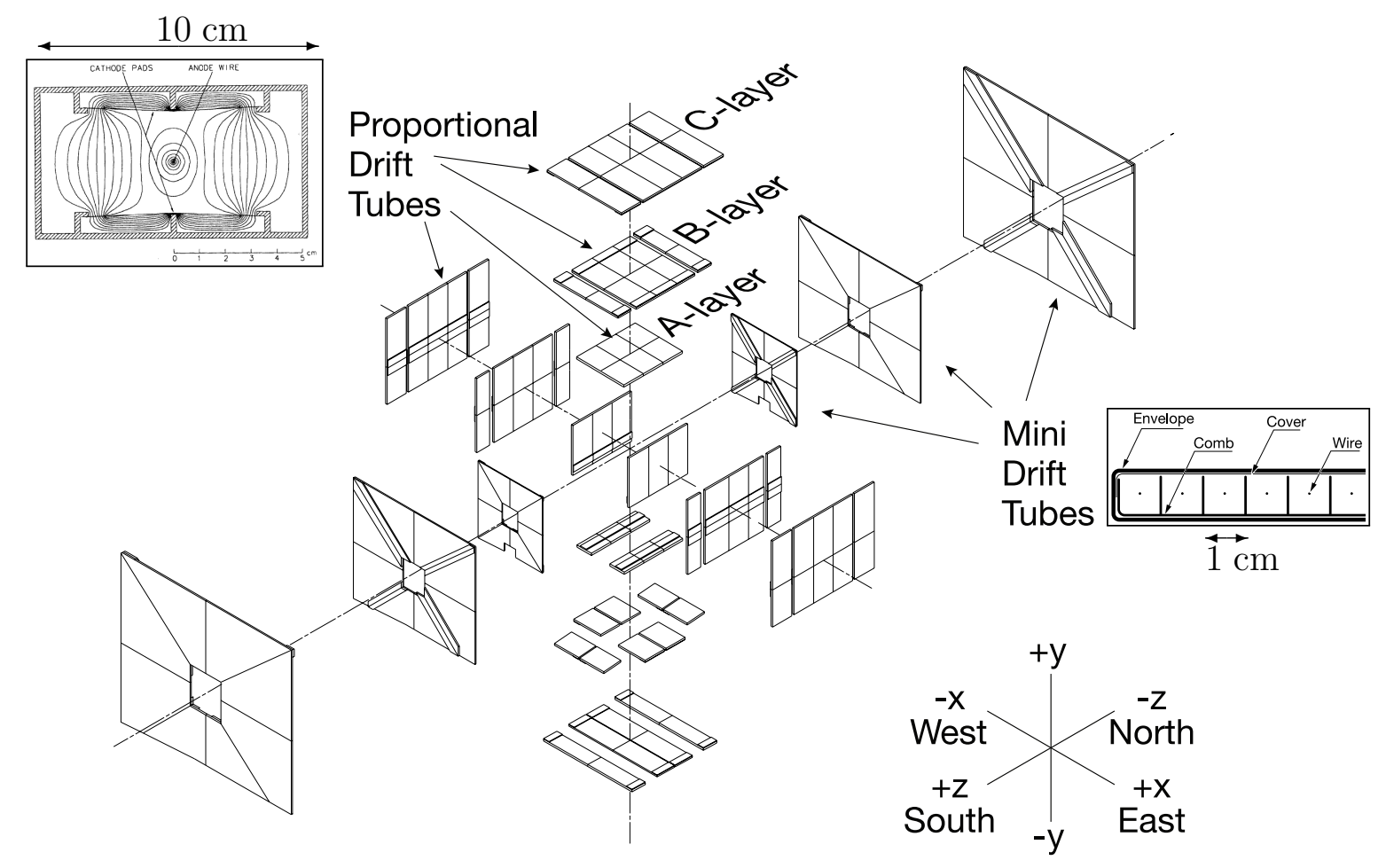

Figure 3.6: Overview of the $\mathrm{D} \varnothing$ muon detector, from Ref. [26]. The layout of the wire chambers is shown. The readout of each layer is subdivided in eight octants, for both FAMUS and WAMUS. Unit cells of the PDT and MDT are illustrated in the two insets. The scintillators, not shown, are arranged in the same rectangular layout.

each reconstructed muon can be unambiguosly associated to a bunch crossing.

\subsubsection{Layout of the Muon Detectors}

The $\mathrm{D} \varnothing$ muon detectors are positioned on planes arranged in a rectangular prism, shown in figure 3.6. The detectors located on the two faces perpendicular to the beam line constitute the forward angle muon system, FAMUS, covering the pseudorapidity region $1 \lesssim|\eta| \lesssim 2$. The detectors located on the other four faces constitute the wide angle muon system, WAMUS, covering the region $|\eta| \lesssim 1$. The supporting structure for the muon detectors is provided by three toroidal magnets, one central and two forward, shown in figure 3.1. Each side of the prism has three layers of muon detectors: one inside the toroids, named A layer, and two outside, named B layer and C layer. Each layer is instrumented with both scintillators and wire chambers. The scintillators' coverage, however, is not uniform on all six sides; 
for example, the bottom side is not fully instrumented due to the presence of the toroid support structures. The wire chambers in the WAMUS are proportional drift tubes; the wire chambers in the FAMUS are mini drift tubes. The drift tubes are arranged in decks, allowing for multiple measurements within each layer, and each layer in subdivided in eight octants. A more detailed description of the muon system can be found in Ref. [26].

\subsubsection{Muon Reconstruction}

A muon is identified by a track reconstructed in the muon system that is matched to an isolated track in the central tracking system. First, track segments are obtained by fitting a straight line to hits from the drift tubes. Two segments are reconstructed: one inside the toroid (fitting hits from the A layer) and one outside the toroid (fitting hits from the B and C layers). The fit $\chi^{2}$, along with the timing information from the scintillators, is used to select the best two segments within each octant. The two segments are then matched with a fit to a local trajectory that takes into account the deflection caused by the $1.8 \mathrm{~T}$ toroidal magnetic field. While the muon curvature is determined during the local track fit, for $p_{\mathrm{T}}$ above $10 \mathrm{GeV}$ the transverse momentum measurement from the central tracker is more precise ${ }^{7}$ than the one from the wire chambers. For the study described here, in which only high- $p_{\mathrm{T}}$ muons are considered, the information from the muon system is only used for identification purposes, and not for the $p_{\mathrm{T}}$ determination. The local muon track is then extrapolated through the calorimeter, accounting for the muon energy due to ionization of the absorber, and fit to a central isolated track. The best fitting central track, that is the one with the minimum matching $\chi^{2}$, is then used to compute the muon quality variables, along with the other pieces of information from the muon system. The muon $p_{\mathrm{T}}$ resolution is shown in figure 3.7.

Reconstructed muons are categorized in classes of different quality: tight, medium, and loose. Each class is defined by the value of the following variables, which are measured either

\footnotetext{
${ }^{7}$ At high momentum, the $p_{\mathrm{T}}$ resolution of the muon system is limited by the individual hit resolution of the wire chambers. At low momentum, it is limited by multiple scattering in the iron of the toroid magnet. Nevertheless, at low momentum the $p_{\mathrm{T}}$ measurement from the muon system is competitive with the one from the central tracker, because the outer measurement is performed over a longer length $L$, see equation (3.2).
} 


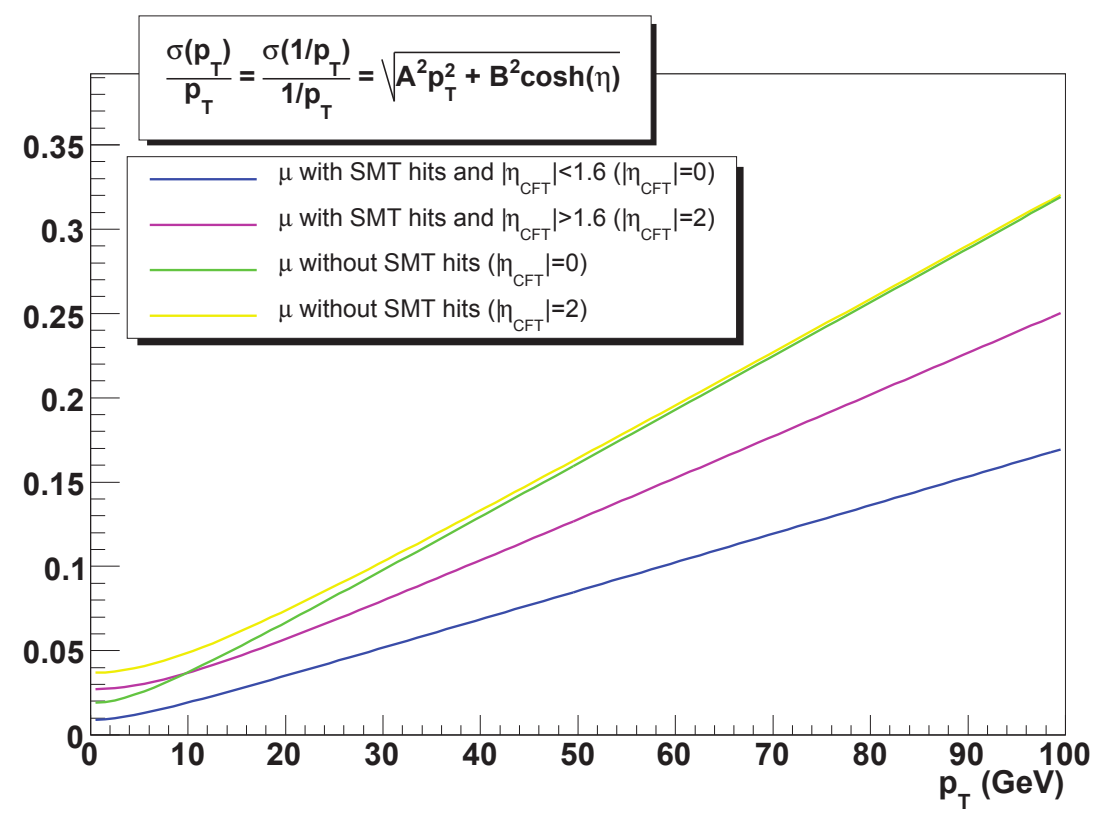

Figure 3.7: The muon transverse momentum resolution, $\quad \sigma_{p_{\mathrm{T}}} / p_{\mathrm{T}}$, for reconstructed muons matching various type of central tracks. From Ref. [37].

in the muon system or in the central tracking system:

- number of segments reconstructed in the muon system, $\mathrm{N}_{\mathrm{seg}}$;

- number of hits in the muon scintillators and wire chambers;

- timing of the scintillator hits;

- for the central track, distance of closest approach (DCA) in the $x-y$ plane with respect to the primary vertex;

- $\chi^{2} / \mathrm{DOF}$ of the central track;

- $\chi^{2} / \mathrm{DOF}$ for the match between the central track and the local muon track;

- calorimeter isolation, computed as the energy deposited in a hollow cone centered around the muon: callso $=E_{\mathrm{T}}^{\mathrm{cal}}(\mathrm{R}<0.4)-E_{\mathrm{T}}^{\mathrm{cal}}(\mathrm{R}<0.1)$, or as calIso scaled $=$ callso $/ p_{\mathrm{T}}^{\mu}$;

- track isolation, computed as the $\mathrm{sum}^{8}$ of the tracks' transverse momenta in a cone centered around the muon: trackIso $=p_{\mathrm{T}}^{\text {tracks }}(\mathrm{R}<0.5)$, or as trackIso ${ }_{\mathrm{scaled}}=\operatorname{trackIso} / p_{\mathrm{T}}^{\mu}$.

\footnotetext{
${ }^{8}$ The muon $p_{\mathrm{T}}$ is not included in this sum.
} 
In this study, we use loose muons, that is muons having at least two drift chamber hits in the A layer and at least two drift chamber hits in the BC layers. Medium muons are loose muons that, in addition, have one matching scintillator in the A layer, and one matching scintillator in the $\mathrm{BC}$ layers. Tight muons are medium muons that, in addition, have a third drift chamber hit in the BC layers, and a track fit that converged. In this study, muons must also have a tracknewmedium track, that is a track with $|\mathrm{DCA}|<0.2 \mathrm{~cm}$ or $|\mathrm{DCA}|<0.04 \mathrm{~cm}$ if there are SMT hits, $\chi^{2} /$ DOF $<9.5$ and at least two CFT hits. In this study, muons must also satisfy the TrkLooseScaled isolation requirement: trackIso scaled $<0.25$, calIso scaled $_{1}<0.4$. A more detailed description of the muon quality criteria can be found in Ref. [38; 39].

\subsection{Missing Energy}

While the momentum of the colliding partons is unknown, its transverse components are in general very small, close to zero. Momentum conservation implies that the sum of the transverse momenta of all the particles produced in a collision is also zero. The production of highly energetic neutrinos in a collision, or of any particle that can escape the detector without being detected, can be inferred by the measurement of an imbalance in the total transverse momentum, usually referred to as "missing transverse energy", MET or $E_{\mathrm{T}}$. Because the $E_{\mathrm{T}}$ is computed by summing the transverse momenta of all objects in the event, its measurement can be performed only after they have all been reconstructed.

\subsubsection{Missing Energy Reconstruction}

The missing energy reconstruction is performed in two main steps. In the first, the vectorial sum of the energies from all calorimeter cells is computed. All of the calorimeter cells with positive energy are used in this step, with the exception of the ones in the $\mathrm{CH}$ section, that have too high a noise level. If the $z$-position of the dilepton pair does not correspond to that of the vertex identified as the primary one, the re-vertexing procedure described in 
section 3.1.3 is performed, and the vectorial sum is updated with the transverse components computed with respect to the dilepton $z_{\ell \ell}$ position.

In the second step, objects that have been fully identified and reconstructed are taken into account. An EM energy scale correction is applied to cells associated to an electron or a photon. Jet energies are corrected for JES, JSSR, in-cone muons originating from $b$-decays, and leakage of hadronic showers in the $\mathrm{CH}$ section of the calorimeter. A $\tau$-energy scale is applied to cells associated with reconstructed taus. Muons that are not associated with jets are taken into account by using their central $p_{\mathrm{T}}$ measurement.

This second step of the $E_{\mathrm{T}}$ reconstruction also gives, as a by-product, another quantity called the unclustered energy, UE. The UE, which is sometimes referred to as LeBOb, Low Energy Background Object, is defined as the vectorial sum of the energy deposits in the calorimeter that are not associated with any reconstructed object. Such deposits can be either due to low- $p_{\mathrm{T}}$ objects produced in zero-bias interactions or to detector effects, such as calorimeter noise. The UE characterizes the calorimetric underlying activity of the event.

\subsection{Trigger}

At the time of writing, the Tevatron has been able to deliver instantaneous luminosities up to $3.7 \times 10^{32} \mathrm{~cm}^{-2} \mathrm{~s}^{-1}$, that is $0.37 \mathrm{nb}^{-1} \mathrm{sec}^{-1}$. While the total $\mathrm{p} \overline{\mathrm{p}}$ cross section is about 0.078 barn (b), the cross sections for interesting processes are much smaller, of the order of $\mathrm{nb}$ and $\mathrm{pb}$. For example, a few $\mathrm{W}$ bosons are produced every second $(\sigma=27 \mathrm{nb})$, a $\mathrm{Z}$ boson is produced every few seconds $(\sigma=8 \mathrm{nb})$, and a few $\mathrm{t} \overline{\mathrm{t}}(\sigma=7.8 \mathrm{pb})$ or diboson $\mathrm{W}^{+} \mathrm{W}^{-}$ $(\sigma=12 \mathrm{pb})$ pairs are produced every hour. If the Higgs boson exists, the cross section for its production at the Tevatron is expected to be less than $1 \mathrm{pb}$, corresponding to several signal events every day.

To reject uninteresting events, the $\mathrm{D} \emptyset$ trigger system relies on the fact that the interesting ones, in which for example a massive particle is produced, show some very distinctive features. 
These signatures include a high- $p_{\mathrm{T}}$ lepton or jet and a large imbalance in the total transverse energy, which might indicate an energetic neutrino. A three-level trigger system is used at $\mathrm{D} \varnothing$ to reduce the event rate from $7.59 \mathrm{MHz}$, which is the detector readout frequency, to $100 \mathrm{~Hz}$, which is the event storage average rate.

At the first level (L1), the information from each subdetector, which is read out every $132 \mathrm{~ns}$, is processed by dedicated hardware. Within $4.2 \mu \mathrm{s}$, the L1 system determines whether one of the 128 unique trigger primitive requirements was satisfied, reducing the event rate to about $2.5 \mathrm{kHz}$. At this stage, the silicon tracker is the only subdetector not to be considered, because the time required for its readout would be too long; the pieces of information from each one of the other subdetectors are read out and processed separately. When an event is accepted at L1, the data, which until now has been kept in the collision hall, is moved to the readout crates. This operation takes $10 \mu \mathrm{s}$, which limits the maximum L1 accept rate to $10 \mathrm{kHz}$.

At the second level (L2), a combined software and firmware system puts together the pieces of information from different subdetectors, and reconstructs primitive physics objects, such as tracks and calorimeter clusters. The L2 decision is made within $100 \mu$ s, and it reduces the event rate to $1 \mathrm{kHz}$.

At the third level (L3), a software program running on a farm of Linux computers performs a simple reconstruction of the event, using the full precision readout of the detector. The reconstruction can take up to $50 \mathrm{~ms}$, and the L3 output rate is about $100 \mathrm{~Hz}$.

Three trigger-related terms often recur when discussing collider experiments. The first one is "prescale". Several types of triggers within a trigger list contribute to the total output rate. Some of them, however, have a much higher rate than others. For this reason, only a predefined fraction of events might be accepted, such as one out of fitfy; in this case, the trigger is said to be prescaled, or to have a prescale. The second and third terms are "zero bias" and "minimum bias". A zero bias event is an event recorded with a random trigger, that is an event of no particular interest. A minimum bias event, or more properly a minimum 
bias scattering, is a collision that was recorded only because it took place in the same bunch crossing in which a hard scattering took place.

\subsection{Event Simulation}

Accurate Monte Carlo (MC) simulations are used in order to make precise predictions about the events that one should expect for the different processes and hypothesized models. Event generators are necessary to perform any measurement at a collider experiment. They simulate, or "produce", events that imitate Nature's behavior in the experiment, and allow for the prediction of the rate and kinematic characteristics of each process under study.

In this section I will summarize the concepts that are relevant to the analysis that will follow; a more detailed overview of this topic can be found in Ref. [40].

\subsubsection{Overview of the Monte Carlo Tools}

Event generators are Monte Carlo software tools that can simulate a complex phenomenon, such as the collision of SM particles, by subdividing into in several, isolated phases. Each phase is ruled by a differerent dynamic, for which an appropriate theoretical or phenomenological description has been developed. This approach is illustrated in figure 3.8, and it can be summarized in three main regimes: hard process, hadronization, and underlying event. These three steps are usually followed by a simulation of the detector acceptance and response.

The hard scattering, in which there is a large-momentum exchange, is usually the process of interest. Colliding hadrons are made of partons, quarks and gluons, each carrying a fraction of the total hadron momentum. This fraction is distributed according to the parton distribution functions, PDF. Inside a hadron, each parton is continuously exchanging gluons, mostly soft and virtual ones, with the other hadron components. Nevertheless, these interactions can be neglected on the timescale of the hard scattering that happens at a higher energy scale and can be described by perturbative calculations. Parton-level event generators can simulate 


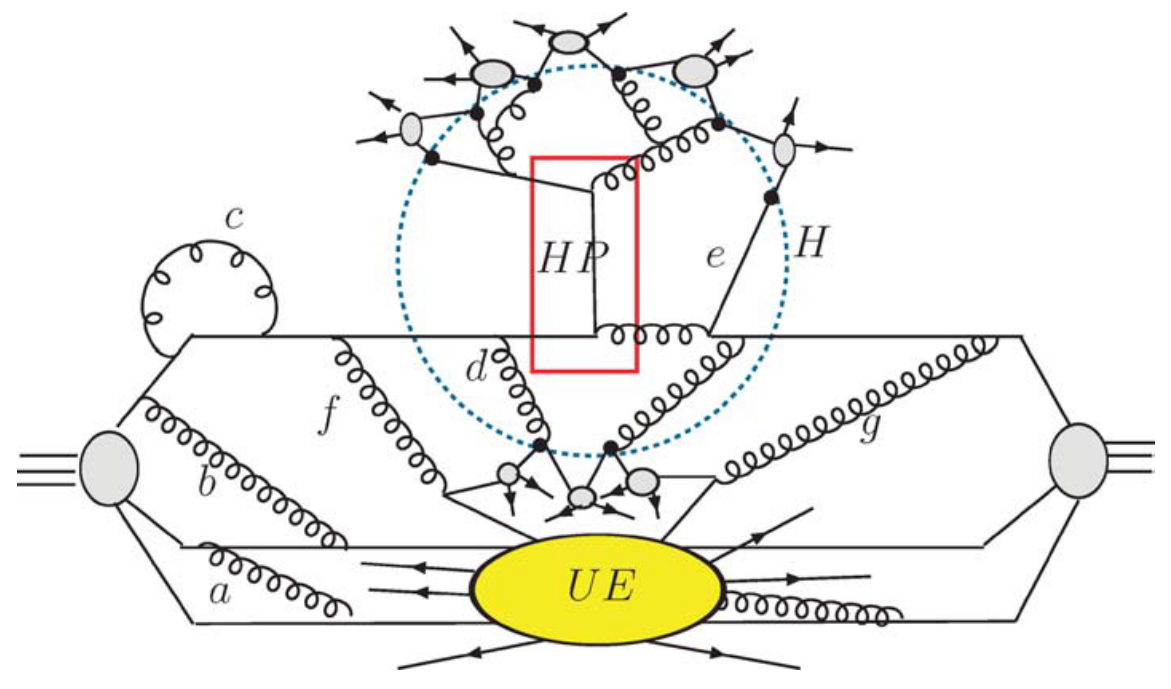

Figure 3.8: Schematic view of a collision between two hadrons, from Ref.[40]. The hard process, indicated with HP, in which two fundamental partons are the main actors, is usually the phenomenon that we want to study. The hadron remnants, which are not involved in the HP, produce the underlying event, indicated with UE. The hadronization takes place after the HP, and evolves until a lower energy scale is reached, indicated by the region labeled as $\mathrm{H}$.

the hard scattering by treating the partons as a set of quasi-free particles. This is done by computing, to the desired level of accuracy in perturbation theory, the corresponding matrix elements convoluted with the PDF. The calculation is usually labeled according to the order at which the matrix elements are computed in perturbation theory, for example at leading order (LO), next-to-leading order (NLO), and next-to-next-to-leading order (NNLO). ALPGEN is one example of a parton-level event generator that can compute at a fixed order, in this case LO, the matrix elements for a large set of processes. It is used in this study to simulate the $\mathrm{Z} / \gamma^{*}(+$ jets $/ \gamma)$ and $\mathrm{W}(+$ jets $/ \gamma)$ processes. The PythiA event generator can be used to simulate a large number of processes as well. As opposed to Alpgen, however, PyтнiA uses a combination of analytical calculations and phenomenological models.

In the following phase, called hadronization, particles that were produced in the hard scattering move apart. If they carry color charge, while moving and accelerating they can also emit additional partons. These colored partons turn into color-less hadrons, due to QCD confinement effects. The hadronization phase is described by phenomenological models, 
implemented for example within the PYTHIA software package.

The underlying event is the set of interactions due to the hadron remnants that are not involved in the hard scattering. Most of these remnants travel in the beam direction without being detected. However, they carry color charge, and therefore have a non-zero probability of interacting with other colored fragments, thus causing additional jets.

Lastly, an important issue, arising from this approach in which the simulation is subdivided in separate phases, should be mentioned. Sometimes, the same hadrons that can be produced within higher-order diagrams can also be generated during the hadronization phase. In this case, special care must be taken to match the color and flavor between the hard scattering phase and that of hadronization. With this matching procedure, one can remove contributions having the same color and flavor, and avoid double counting.

\subsubsection{Usage of Monte Carlo Tools at DØ}

Pythia and Alpgen are the two event generators used in this analysis to simulate the hard scattering. While PyтніA can simulate both the hard scattering and the hadronization steps, AlPGen can only compute the matrix elements. Events generated with AlPGEN are therefore subsequently processed by PүтнIA to simulate the hadronization and the decay of short-lived particles. In this case, in order to avoid the double countings described in the section 3.6.1, the matching procedure is performed, followed by heavy-flavor removal described in Ref. [41; 42].

The particles produced during the three simulation steps described in section 3.6.1 are then propagated through a model of the $\mathrm{D} \varnothing$ detector. In this stage, the GEANT software package [43] is used to determine the effects of magnetic fields and multiple scattering on the particles' trajectories, as well as the energy deposited in each part of the detector. The energy deposited in the instrumented parts of the detector is then converted into expected readout values. Zero bias data events are overlayed to these simulated readout values, to account for effects such as calorimeter noise and pile up. The resulting simulated events are 
then processed with the same reconstruction algorithms used for data events.

The normalization of each MC samples is computed using equation (3.4), where $\mathrm{L}$ is the dataset integrated luminosity, $\sigma$ is the cross section for the corresponding process, and $N_{\mathrm{MC}}$ is the number of $\mathrm{MC}$ events being used.

$$
\frac{\sigma \times \mathrm{L}}{N_{\mathrm{MC}}}
$$

Object reconstruction efficiencies for data and MC events are taken into account in the MC event weight used for normalization purposes. Furthermore, when the resolution of a quantity measured in $\mathrm{MC}$ events is better than the one observed in data, additional MC corrections are applied.

For some of the processes, the hard scattering simulation is performed at LO, giving the differential spectra at LO. Nevertheless, calculations of the corresponding cross section are sometimes available with higher-order accuracy. In such cases, the event rate is therefore adjusted, and the normalization is adjusted by a correction factor $\sigma_{\text {higher order }} / \sigma_{\mathrm{LO}}$, which is called ${ }^{9} k$-factor. The value of the $k$-factor can vary depending on the quark flavor content, resulting in different $k$-factors for final states with heavy quarks, such as $\mathrm{c}$ and $\mathrm{b}$.

\footnotetext{
${ }^{9}$ The name $k$-factor originates from the fact that the ratio of the corrected and LO cross sections is almost constant in rapidity, as described in Ref. [44]
} 


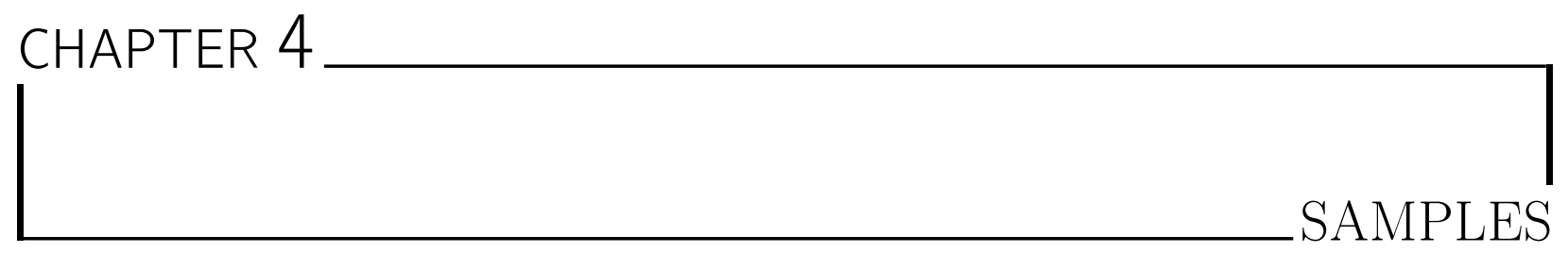

This chapter illustrates the event samples used in the analysis. Section 4.1 provides a description of the dataset used. In section 4.2 , the simulated samples used to predict the signal and background contributions are described. Section 4.3 describes the requirements that the events have to satisfy in order to be considered in the subsequent steps of the analysis.

The Higgs boson search described in this thesis is performed in two configurations. In the first configuration, we use a neural network (NN) discriminant, and analyze a dataset of $5.4 \mathrm{fb}^{-1}$ collected from April 2002 through June 2009. We refer to this study as the "NN analysis". In the second configuration, we use a decision tree (DT) discriminant, and analyze a dataset of $8.1 \mathrm{fb}^{-1}$ which supersedes the previous one by including the additional $2.7 \mathrm{fb}^{-1}$ of data collected through December 2010. We refer to this study as the "DT analysis". Most of the selection criteria and samples are common to the NN study and the DT study; we will mention the minor differences between the two studies in the following sections. On the contrary, the two procedures used in the two analyses present significant differences. These two procedures are described in chapter 5, and illustrated in figure 5.1. 


\subsection{Data sample}

The 8.1- $\mathrm{fb}^{-1}$ dataset recorded by the DØ detector between April 2002 and December 2010 is called the RunII dataset. The Tevatron RunII is currently ongoing, and the RunII dataset growing. The Tevatron RunII is scheduled to end in September 2011; at that time, the data collected by the DØ experiment will amount to $11 \mathrm{fb}^{-1}$. Since the start of RunII, the detector configuration has changed, most notably with the introduction of the SMT "layer 0" in the summer of 2006. For this reason, the RunII dataset is usually subdivided into RunIla, which corresponds to the $1.1 \mathrm{fb}^{-1}$ recorded before the upgrade, and RunIIb, which corresponds ${ }^{1}$ to the $7.0 \mathrm{fb}^{-1}$ recorded after the upgrade.

The RunII dataset is reduced by the $\mathrm{D} \varnothing$ collaborators to smaller subsets with specific final states, called "skims." In this analysis, we used the 2MuHighPt skim, which contains more than 120 million events with two very loosely reconstructed muons that have a $p_{\mathrm{T}}>10 \mathrm{GeV}$. The requirements that we apply, in addition to the presence of two muons, are illustrated in the following two sections, which describe the trigger and the data quality.

Trigger We do not require the events to have fired any specific trigger. This allows us to use those events in which two muons are reconstructed offline, even though the events are not recorded because of a muon trigger, but rather because of other triggers.

Previous strategies [45; 46] for analyses involving dimuon final states relied on requiring the logic OR of multiple single-muon triggers; this approach is usually referred to as SingleMuonOR. Although the acceptance that can be achieved with the SingleMuonOR approach is significanlty higher than the one that can be achieved with a single muon trigger, its efficiency is still limited. The SingleMuonOR efficiency, which involves contributions from several triggers with different prescales, has been estimated in Ref. [45; 46] using the tag-and-probe method.

With respect to the SingleMuonOR strategy, the inclusive approach used in this analysis gives almost a $20 \%$ increase [47] in the number of data events that can be analyzed. The

\footnotetext{
${ }^{1}$ The RunIIb dataset was $4.3 \mathrm{fb}^{-1}$ for the NN study; the RunIIb dataset was $7.0 \mathrm{fb}^{-1}$ for the DT study.
} 
additional contributions are mostly due to the Muon+Jets triggers, and the fraction of additional events depends on the number of jets in each of them. This requires the normalization and $\mathrm{MC}$ correction procedures described in chapter 5.

Data quality and Luminosity The quality of the data is continously monitored by the operations crew while the data is being taken. For every run, any malfunction in any of the subdetector systems is logged. In addition, a more detailed assessment of the data quality (DQ) is performed offline by the DØ DQ group [48]. In addition to being more accurate than the online assessment, the offline DQ is determined on a finer timescale, in which the base unit is a "luminosity block" corresponding to about four minutes of data taking. Within a data-taking run, four minutes also correspond to the unit of time over which the instantaneous luminosity is determined by averaging multiple detector readings.

The dataset integrated luminosity is measured by summing the instantaneous luminosity of all the valid luminosity blocks. Its absolute normalization is computed with respect to the total inelastic cross section for $\mathrm{p} \overline{\mathrm{p}}$ at $1.96 \mathrm{TeV}$ by counting the number of events that fire the experiment's highest $E_{\mathrm{T}}$ jet trigger. This trigger requires at least one jet with $E_{\mathrm{T}}>125 \mathrm{GeV}$ and is never prescaled [49;50]. The resulting uncertainty on the dataset integrated luminosity is $6.7 \%$.

\subsection{Monte Carlo Samples}

For all signal processes and for most of the background ones, we use MC simulated samples to determine the expected number of events and their kinematic characteristics. As described in section 5.2, only the background component due to multijet production is estimated from data. 


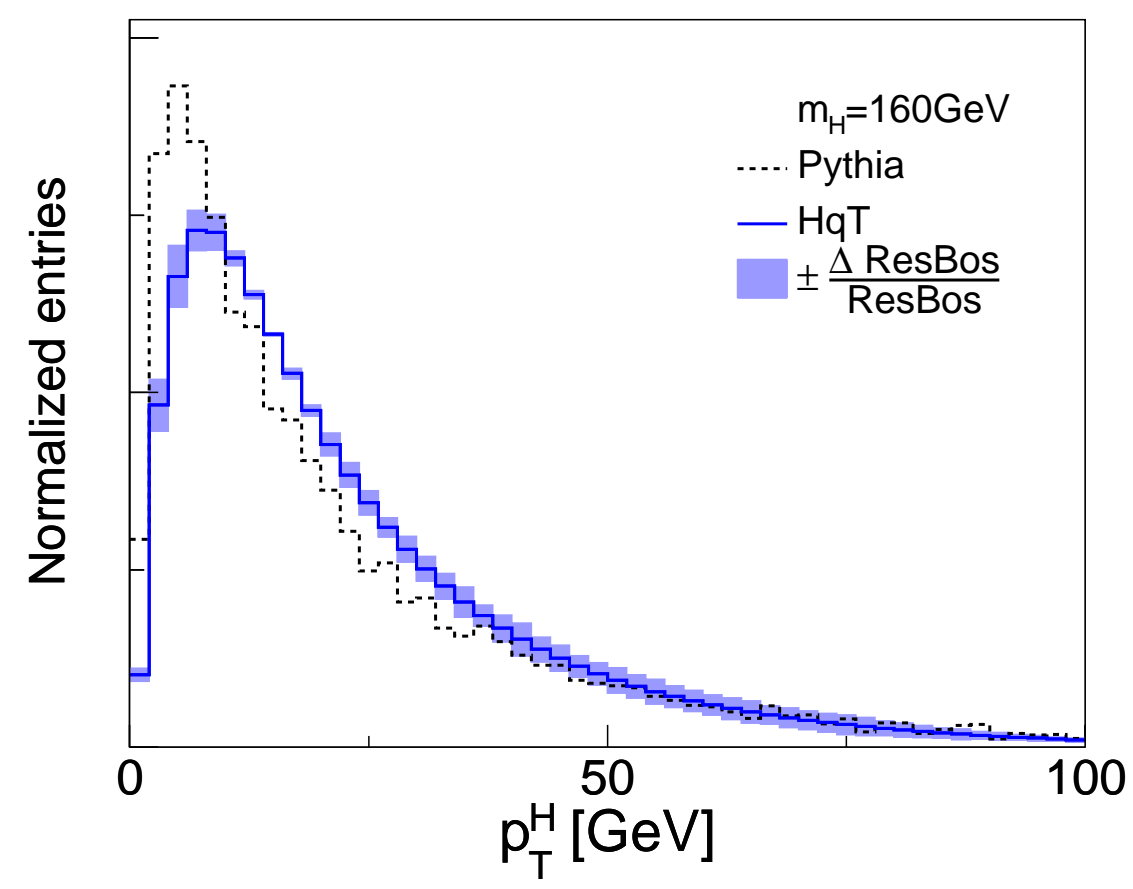

Figure 4.1: Higgs boson transverse momentum: comparison of the LO prediction by PYTHIA with the NLO prediction by HQT [60]. The gg $\rightarrow \mathrm{H}$ samples generated with PYTHIA are reweighted to match the harder $p_{\mathrm{T}}^{\mathrm{H}}$ spectrum predicted at NLO. The HQT calculation also includes the resummation of logarithmicallyenhanced contributions at second order in QCD perturbations, NNLL.

\subsubsection{Signal Samples}

The signal samples are generated at LO with PYTHIA ${ }^{2}$ [51] using the CTEQ6L [52] parton distribution functions. We consider the following Higgs production mechanisms: gluon-gluon fusion $(\mathrm{gg} \rightarrow \mathrm{H})$, associated production $(\mathrm{WH}$ and $\mathrm{ZH})$, and vector boson fusion $(q q \rightarrow q q \mathrm{H})$. The signal cross sections are calculated for different Higgs boson masses at NNLO [53] as listed in Ref. [54-59], and at NLO in the case of the vector boson fusion process. In addition, the spectrum of the Higgs boson transverse momentum in $\mathrm{gg} \rightarrow \mathrm{H}$ samples is reweighted to match the more accurate NLO predictions, as illustrated in figure 4.1. Despite the fact that the $p_{\mathrm{T}}$ of the Higgs boson, $p_{\mathrm{T}}^{\mathrm{H}}$, is not measurable in the $\bar{\ell} \nu \ell \bar{\nu}$ final state, this reweighting is applied in order to improve the modeling of those observables that depend on $p_{\mathrm{T}}^{\mathrm{H}}$, such as the dilepton $p_{\mathrm{T}}$ and the angular separation between the two leptons. The Higgs boson branching ratios are determined at NLO using HDECAY [20].

For the $\mathrm{gg} \rightarrow \mathrm{H}$ process, samples are generated considering the following decays: $\mathrm{H} \rightarrow$ $\mathrm{W}^{+} \mathrm{W}^{-} \rightarrow \bar{\ell} \nu \ell \bar{\nu}$, and $\mathrm{H} \rightarrow \mathrm{ZZ}$, where $\mathrm{ZZ} \rightarrow \ell \bar{\ell} \ell \bar{\ell}, \mathrm{ZZ} \rightarrow \ell \bar{\ell} j j$, or $\mathrm{ZZ} \rightarrow \ell \bar{\ell} \nu \bar{\nu}$. For the other

\footnotetext{
${ }^{2}$ Version 6.323 and 6.409 is used.
} 
signal processes, the $\mathrm{H} \rightarrow \mathrm{W}^{+} \mathrm{W}^{-} \rightarrow \bar{\ell} \nu \ell \bar{\nu}$ is considered.

Signal samples are generated for hypothetical mass values $\left(\mathrm{m}_{\mathrm{H}}\right)$ in the range $115-200 \mathrm{GeV}$ with $5 \mathrm{GeV}$ increments. For the fourth-generation study described in section 6.3.2, additional gg $\rightarrow \mathrm{H}$ samples are generated in the range $210-300 \mathrm{GeV}$ with $10 \mathrm{GeV}$ increments. Numerical values of the signal cross section can be found in table A.1; numerical values of the branching fractions can be found in table A.2.

\subsubsection{Background Samples}

Within the standard model, several processes not involving the production of a Higgs boson can produce events with two high- $p_{\mathrm{T}}$ leptons and missing transverse energy. These backgrounds processes are usually grouped in two categories: intrinsic backgrounds and instrumental backgrounds.

Intrinsic backgrounds are SM processes with a genuine dilepton plus missing energy final state. Examples of such background processes include: diboson production, comprehending

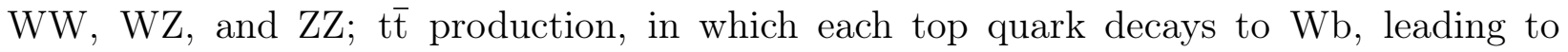
$\mathrm{t} \overline{\mathrm{t}} \rightarrow \ell \nu \ell \nu \mathrm{b} \overline{\mathrm{b}}$.

Instrumental backgrounds are SM processes with a final state that is not exactly the same as the signal one. Nevertheless, because of the detector reconstruction effects or the misidentification of one of the particles, events due to instrumental backgrounds look like signal events. Examples of such backgrounds include: $\mathrm{Z} / \gamma^{*}(+$ jets $/ \gamma)$ events, in which a poorly reconstructed lepton $p_{\mathrm{T}}$ can mimic an imbalance in the transverse energy; $\mathrm{W}(+$ jets $/ \gamma)$ events, in which a jet or photon is misidentified as a lepton; multijet events in which jets are misidentified as leptons or contain muons produced by hadron decays within the shower.

As mentioned at the beginning of section 4.2, only the multijet background is estimated from data, whereas all the other backgrounds are estimated with MC simulations. We use the ALPGEn [61] event generator ${ }^{3}$ to model the $\mathrm{Z} / \gamma^{*}(+$ jets $/ \gamma)$ and $\mathrm{W}(+$ jets $/ \gamma)$ backgrounds,

\footnotetext{
${ }^{3}$ Version 2.11 was used.
} 
and Pythia [51] for the diboson and $t \bar{t}$ ones. The expected rate for $\mathrm{Z} / \gamma^{*}(+\mathrm{jets} / \gamma)$ and $\mathrm{W}(+\mathrm{jets} / \gamma)$ is normalized by applying the appropriate $k$-factor to the NNLO calculation of Ref. [62], performed using the MRST 2004 NNLO PDF set [63]. The corresponding numerical values can be found in tables A.3 to A.5. The expected rate for tt production is normalized to the NNLO calculation from Ref. [64], and the ones for WW, WZ, and ZZ are normalized to the NLO calculation from Ref. [65]. The corresponding numerical values can be found in table A.6.

\subsection{Preselection}

This search for the SM Higgs boson is performed using events that are reconstructed in a final state consistent with the one expected for $\mathrm{H} \rightarrow \mathrm{W}^{+} \mathrm{W}^{-} \rightarrow \bar{\ell} \nu \ell \bar{\nu}$ potential signal events. Therefore, before performing any analysis of the data, we select events with at least two reconstructed leptons. This section describes the set of initial selection criteria that we apply to the data and $\mathrm{MC}$ samples after the $\mathrm{DQ}$ requirements. The events that satisfy these criteria constitute what is often referred to as the "preselection" stage. The preselection requirements are similar for the three channels $\left(\mathrm{e}^{+} \mathrm{e}^{-}, \mathrm{e}^{ \pm} \mu^{\mp}\right.$, and $\left.\mu^{+} \mu^{-}\right)$in which the $\mathrm{D} \emptyset \mathrm{H} \rightarrow \mathrm{WW}$ search is performed. We will only address the dimuon ones here.

The events considered in this study have at least two muons with high transverse momentum. Additional reconstructed leptons are disregarded. Throughout the following chapters, we refer to the muon with the highest $p_{\mathrm{T}}$ as $\mu_{1}$, and to the one with the second-highest $p_{\mathrm{T}}$ as $\mu_{2}$. We require that $p_{\mathrm{T}}^{\mu_{1}}>20 \mathrm{GeV}$ and $p_{\mathrm{T}}^{\mu_{2}}>10 \mathrm{GeV}$. In the DT analysis, the $p_{\mathrm{T}}^{\mu_{1}}>20 \mathrm{GeV}$

requirement is replaced by $p_{\mathrm{T}}^{\mu_{1}}>15 \mathrm{GeV}$, allowing for the recovery of up to $4 \%$ of signal events at low mass, as illustrated in figure 4.2.

We select muons that have been reconstructed with the criteria listed in table 4.1, established by the MuonID group, and described in section 3.3.2. For signal events, we expect the two muons to be collinear, as shown in figure 4.3. The TrkLooseScaled isolation 

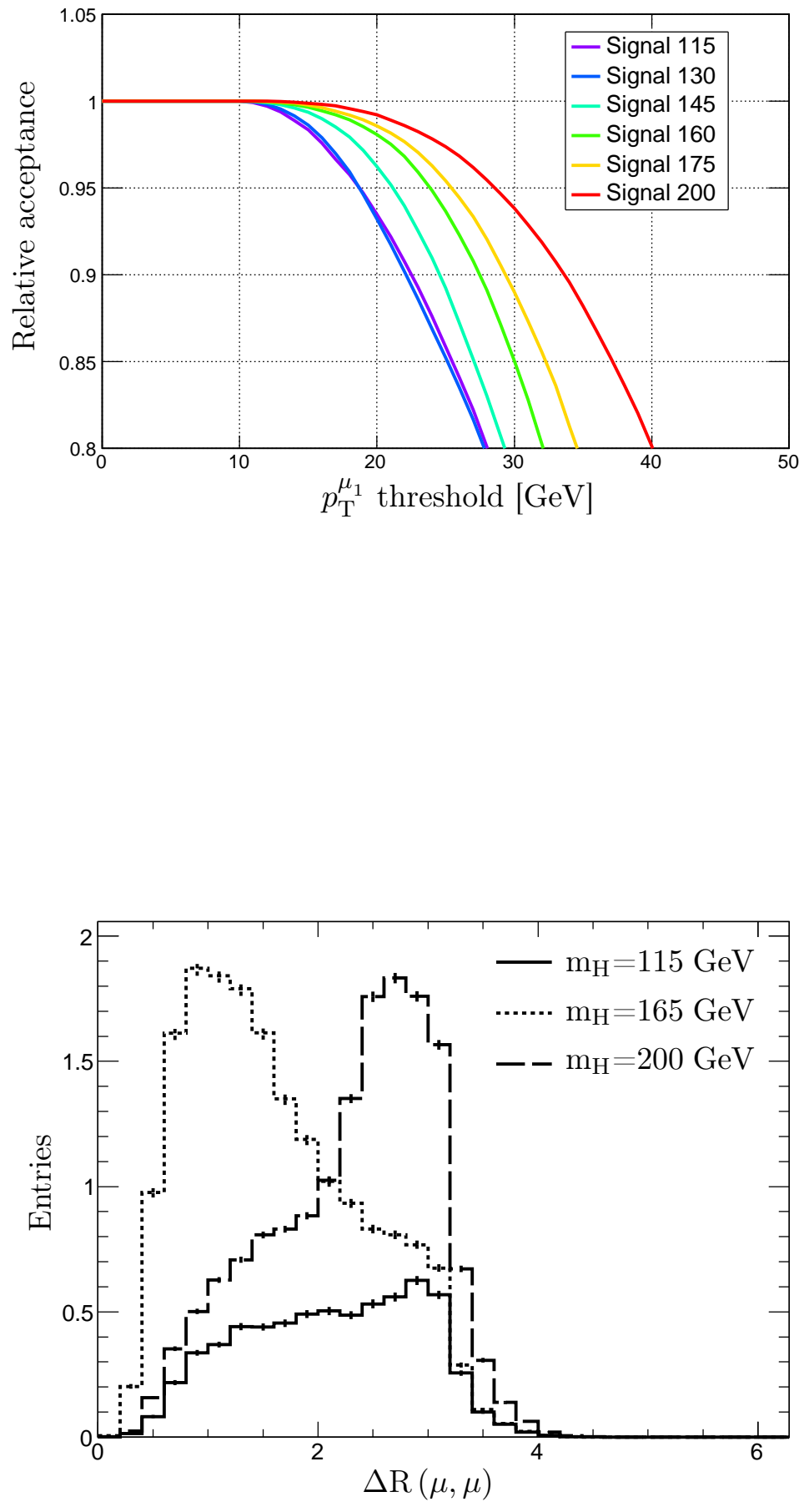

Figure 4.2: Signal acceptance as a function of the $p_{\mathrm{T}}^{\mu_{1}}$ minimum threshold, shown for several $\mathrm{m}_{\mathrm{H}}$ values. The acceptance is relative to the $p_{\mathrm{T}}^{\mu_{1}}>10 \mathrm{GeV}$ criterion.

Figure 4.3: Signal events: angular separation $\Delta \mathrm{R}(\mu, \mu)$ for several $\mathrm{m}_{\mathrm{H}}$ values at preselection stage. Dimuon pairs with small separation, namely with $\Delta R<0.5$, would fail the TrkLooseScaled isolation requirement. The modified isolation requirement used in this study can recover these signal events. The fraction of signal events that are recovered is different for different values of $\mathrm{m}_{\mathrm{H}}$. 


\begin{tabular}{lr}
\hline \multicolumn{2}{c}{ Muon reconstruction criteria } \\
\hline Muon quality & loose \\
Central track quality & newmedium \\
Muon Isolation & TrkLooseScaled \\
\hline
\end{tabular}

Table 4.1: Muon reconstruction criteria; each criterion is described in section 3.3.2.

criterion requires that calIso scaled $<0.4$ and that trackIso scaled $<0.25$, however. Therefore, a non-negligible fraction of signal events would be lost, due to the fact that, in a pair of collinear muons, the $p_{\mathrm{T}}$ of one muon is accounted for when computing the isolation of the other muon. In this study, we modify the isolation requirement so that the $p_{\mathrm{T}}$ contribution due to the other lepton in the dilepton pair is not considered when summing the $p_{\mathrm{T}}$ of the tracks in the cone. Up to $3 \%$ of the signal events are recovered, with the fraction of recovered signal events varying for different values of $\mathrm{m}_{\mathrm{H}}$.

In addition to the MuonID criteria, we apply the following requirements, which are specific to $\mathrm{H} \rightarrow \mathrm{W}^{+} \mathrm{W}^{-} \rightarrow \mu^{+} \nu \mu^{-} \bar{\nu}$ events and summarized in table 4.2: the two muons must correspond to the same hard-scattering event, and are therefore required to originate from the same location. This translates into one of the following two constraints: either the two muons are associated with the same primary vertex, or they have the same $z$ coordinate at $(x, y)=$ (beamspot location). The two muons must have high transverse momenta. They must have opposite charge (but this requirement is reversed for the multijet estimation described in section 5.2). Their invariant mass $M_{\mu \mu}$ must be above $15 \mathrm{GeV}$, in order to avoid contributions from low-mass resonances (mainly $\mathrm{J} / \psi$ and $\Upsilon$ ). To avoid contaminations from muons due to in-flight hadronic decays, they must be well separated from any jet in the event. In addition to the TrkLooseScaled conditions, they must also satisfy tighter isolation requirements. For the NN analysis, we require that the total scaled isolation,

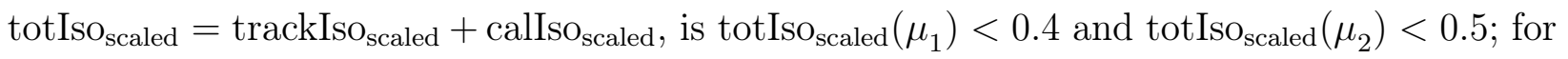
the DT analysis, we require that the absolute calorimeter isolation be less than $10 \mathrm{GeV}$ for both muons. 
Table 4.2 indicates, for the $5.4 \mathrm{fb}^{-1} \mathrm{NN}$ analysis and for the $8.1 \mathrm{fb}^{-1} \mathrm{DT}$ analysis, the exact thresholds for each one of the above requirements. There are only minor differences between the two sets of preselection criteria: the $p_{\mathrm{T}}^{\mu_{1}}$ threshold for the $8.1 \mathrm{fb}^{-1} \mathrm{DT}$ analysis is lower than that used for the $5.4 \mathrm{fb}^{-1} \mathrm{NN}$ analysis; the isolation requirement is changed from the scaled isolation to a calorimeter isolation, which is more robust at high instantaneous luminosity; the requirement that the two leptons originate from the same vertex is replaced by a $\Delta\left(z_{\mu 1}, z_{\mu 2}\right)$ selection, allowing for the inclusion of signal events in which the PV identification algorithm failed.

Table 4.2: Preselection requirements.

\begin{tabular}{|c|c|c|}
\hline & NN $5.4 \mathrm{fb}^{-1}$ analysis & DT $8.1 \mathrm{fb}^{-1}$ analysis \\
\hline Dimuon invariant mass & \multicolumn{2}{|c|}{$M_{\mu \mu}>15 \mathrm{GeV}$} \\
\hline Transverse momentum $\mu_{1}$ & $p_{\mathrm{T}}^{\mu_{1}}>20 \mathrm{GeV}$ & $p_{\mathrm{T}}^{\mu_{1}}>15 \mathrm{GeV}$ \\
\hline Transverse momentum $\mu_{2}$ & \multicolumn{2}{|c|}{$p_{\mathrm{T}}^{\mu_{2}}>10 \mathrm{GeV}$} \\
\hline Isolation $\mu_{1}$ & totIsO scaled $\left(\mu_{1}\right)<0.4$ & $\operatorname{calIso}\left(\mu_{1}\right)<10 \mathrm{GeV}$ \\
\hline Isolation $\mu_{2}$ & $\operatorname{totIsO}_{\text {scaled }}\left(\mu_{2}\right)<0.5$ & callso $\left(\mu_{2}\right)<10 \mathrm{GeV}$ \\
\hline Muon separation from jets & \multicolumn{2}{|c|}{$\min \left(\Delta R\left(\mu_{1}\right.\right.$, jet $), \Delta R\left(\mu_{2}\right.$, jet $\left.)\right)>0.1$} \\
\hline Track origin & Shared PV, $\left|z_{\mathrm{PV}}\right|<60 \mathrm{~cm}$ & $\left|\Delta\left(z_{\mu 1}, z_{\mu 2}\right)\right|<2 \mathrm{~cm}$ \\
\hline Central track match & - & matching $\chi^{2}<100$ \\
\hline
\end{tabular}




\section{CHAPTER 5 ANALYSIS}

In chapter 4 we defined the data, signal, and background samples used in this thesis; chapter 5 addresses the analysis of the data. It describes the methods used to separate the background events from the candidate signal events, and to construct a variable to test the Higgs boson hypothesis.

The first step of the data analysis consists in normalizing the event yields. The normalization of the number of MC event is performed at preselection and is described in section 5.1.

The second step of the analysis sets out to estimate the multijet background. Its contribution is determined from the sample of events that have two muons with the same electric charge, as described in section 5.2. Corrections to the Monte Carlo samples are then applied, as described in section 5.3.

The third step aims to reduce the number of $\mathrm{Z} / \gamma^{*}(+$ jets $/ \gamma)$ events. The $\mathrm{Z} / \gamma^{*}(+$ jets $/ \gamma)$ background, often referred to as the Drell-Yan (DY) background, has the greatest event yield at preselection: there are at least 29 thousands DY events for each signal event, at any $\mathrm{m}_{\mathrm{H}}$ value under consideration.

In the fourth step of the data analysis, we construct a multivariate discriminant to separate 


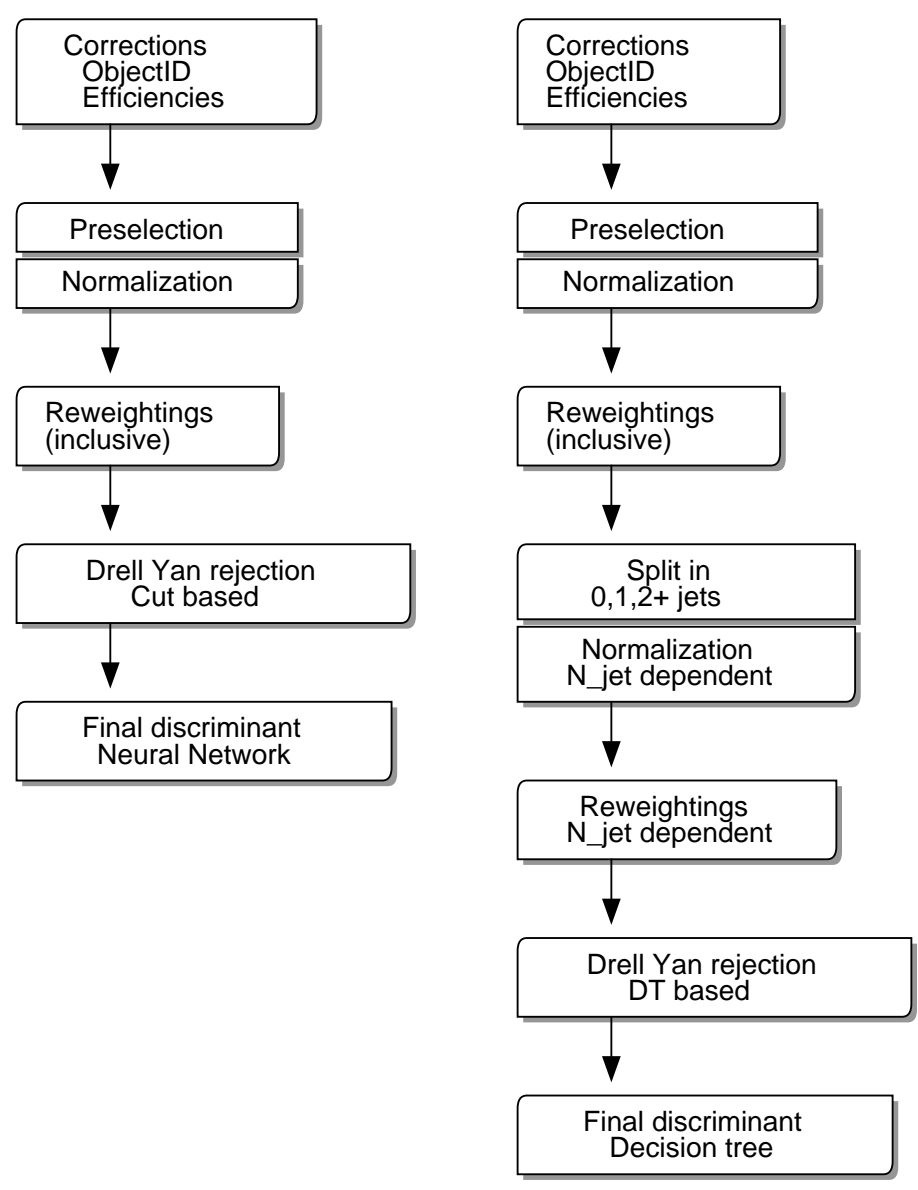

Figure 5.1: Overview of the analysis workflow for the NN study (left) and for the DT study (right).

candidate signal events from those produced by all backgrounds, including the DY one. We then use the output of this final discriminant to test the Higgs boson hypothesis. Section 5.6 describes the multivariate discriminants for the NN study, neural networks, and for the DT study decision trees.

In section 5.5, we describe two methods designed to reject DY events. The first method, based on kinematic selections, is used in the $5.4 \mathrm{fb}^{-1} \mathrm{NN}$ study. The second method, based on a multivariate discriminant, is used in the $8.2 \mathrm{fb}^{-1}$ DT study. Both methods use several event variables, and most of these variables are common to the two methods. In addition to being used to reject DY events, several of these variables are also inputs to the final discriminants. Before illustrating the DY rejection in section 5.5, and the final discriminant in section 5.6, we describe all the input variables in section 5.4.1. 


\subsection{Normalization}

The MC samples are normalized through a two-step procedure performed at the preselection stage, in which the total event yield is dominated by the $\mathrm{Z} / \gamma^{*}(+$ jets $/ \gamma)$ background. For both steps, our normalization procedure is based on counting the number of events in the $\mathrm{Z}$ peak. An important feature of this normalization procedure is that the luminosity uncertainty, along with part of uncertainties due to trigger and lepton ID efficiencies, are now included in the normalization uncertainty.

In the first step, a surface normalization is calculated to account for any MC mismodeling of lepton ID, trigger efficiency, or luminosity. This normalization factor is calculated as follows:

$$
S=\frac{N_{\text {data }}}{N_{M C}} \text {, for } 80 \mathrm{GeV}<M_{\ell \ell}<100 \mathrm{GeV}
$$

The $S$ factor, reported in table 5.1 for the RunIIa and RunIIb epochs, is applied to all signal and background MC samples. Figure 5.2 shows the dimuon invariant mass distributions after the $S$ factor is applied. The $15 \%$ deviation from unity of the $S$ factor in the $\mu \mu$ final state is mainly due to the lack of any trigger requirement for the MC.

In the second step, a jet-multiplicity-dependent normalization factor is computed. This second step is performed for the DT study only and not for the NN study. The jet-multiplicitydependent factor $\alpha_{i-j e t}$ is computed after applying the inclusive surface normalization $S$ :

$$
\alpha_{i-j e t}=\frac{N_{d a t a}^{i-j e t}}{S \times N_{M C}^{i-j e t}} \text { for } 80 \mathrm{GeV}<M_{\ell \ell}<100 \mathrm{GeV}
$$

with $i-j e t$ equal to the $0-j e t$ exclusive, $1-j e t$ exclusive, and $2-j e t$ inclusive samples. This cross-section correction is applied solely to the $Z+$ jets MC samples, and the values of these corrections are listed in table 5.1. The statistical error on these factors is less than $1 \%$, but there is a larger systematic uncertanity associated with the choice of $\mathrm{Z}$ mass window. The deviation from unity of the $\alpha_{2-j e t}$ factors can be attributed to the generator-based mismodellings of the Alpgen $Z+$ jets samples. 


\begin{tabular}{ccc}
\hline & \multicolumn{2}{c}{$\mu \mu$} \\
\cline { 2 - 3 } & RunIIa & RunIIb \\
\hline Surface Norm. $(S)$ & 0.85 & 0.99 \\
\hline$\alpha_{0-\text { jet }}$ & 1.00 & 0.99 \\
$\alpha_{1-\text { jet }}$ & 0.95 & 1.02 \\
$\alpha_{2-\text { jet }}$ & 1.14 & 1.21 \\
\hline
\end{tabular}

Table 5.1: Table summarising the surface normalization factors $S$, and jet-multiplicitydependent corrections $\alpha_{i-j e t}$

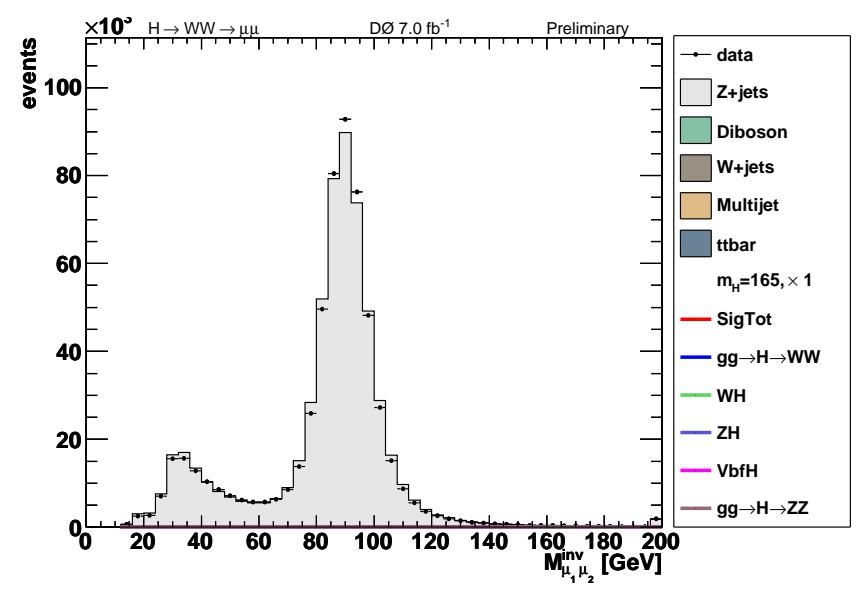

Figure 5.2: Distribution of the dimuon invariant mass for the RunIIb period after the surface normalization $S$ is applied.

\subsection{Multijet Estimate}

The multijet background is estimated using data events in which the two muons have the same charge, under the assumption that multijet events are equally likely to mimic same-sign dimuon pairs and opposite-sign dimuon pairs. The number of same-sign events predicted by the Monte Carlo simulation is scaled by the normalization factor $S$, which is obtained from the opposite sign sample, as described in section 5.1. The normalization factor $S$ is applied under the assumption that $S$ accounts mostly for trigger effects, and that these effects have the same magnitude for same-sign events and for opposite-sign events. We then consider the $p_{\mathrm{T}}$ distributions for the two muons in the same-sign sample, shown in figure 5.3, in which the missing contribution due to multijet is visible. On the contrary, the 
multijet contribution is negligible at the preselection stage for the opposite-sign sample. The quantity $($ data $-M C) /$ data is then computed for each bin, and fitted with a decreasing exponential. Following this procedure, we obtain two scale functions $e_{1}\left(p_{\mathrm{T}}^{\mu_{1}}\right)$ and $e_{2}\left(p_{\mathrm{T}}^{\mu_{2}}\right)$, shown in figure 5.4. The sign of the second lepton for events from the same-sign data sample
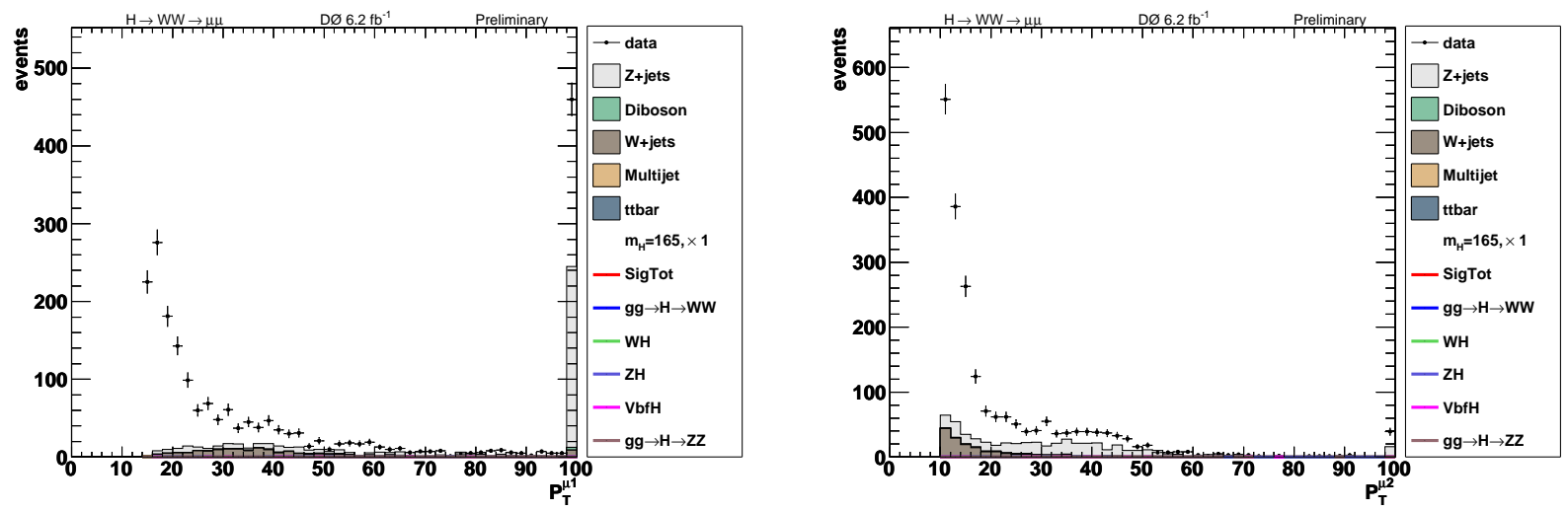

Figure 5.3: Transverse momenta distributions of the two muons for like-sign events for the RunIIb epoch.
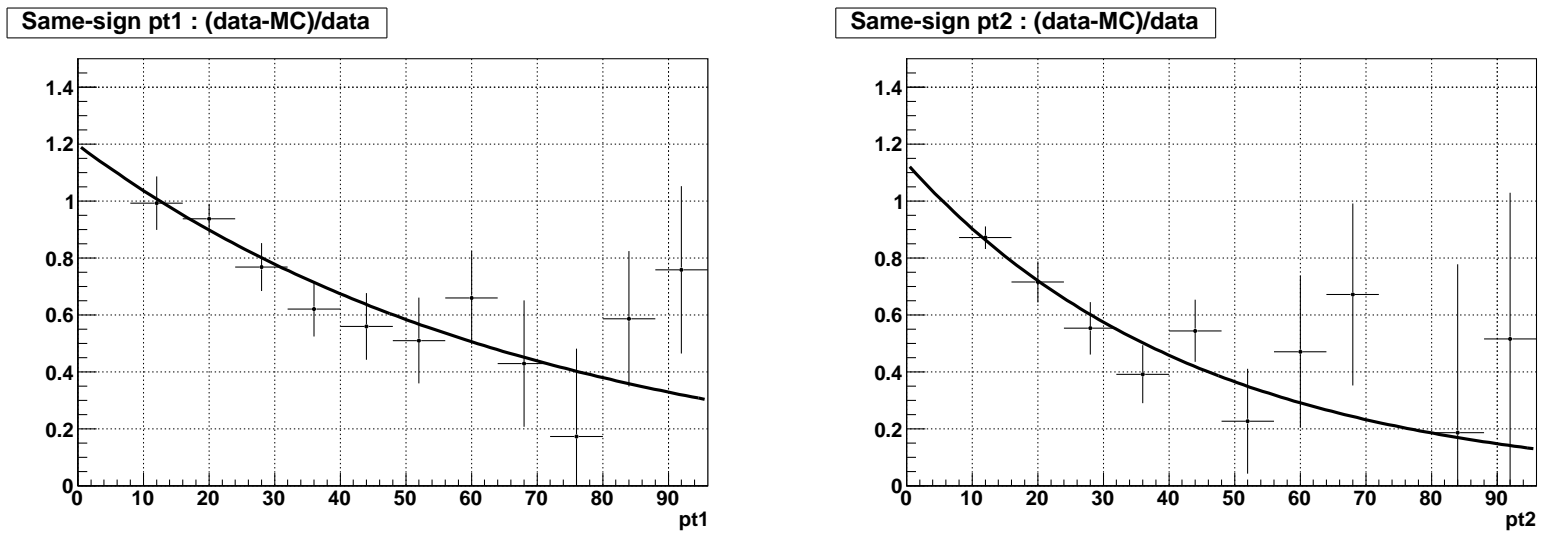

Figure 5.4: Fit to the di-muon multijet scale factors $e_{1}\left(p_{\mathrm{T}}^{\mu_{1}}\right)$ and $e_{2}\left(p_{\mathrm{T}}^{\mu_{2}}\right)$ for the RunIIb epoch.

is then inverted, and the event weight is set to the average:

$$
\frac{e_{1}\left(p_{\mathrm{T}}^{\mu_{1}}\right)+e_{2}\left(p_{\mathrm{T}}^{\mu_{2}}\right)}{2} .
$$

The resulting set of opposite-sign events constitutes our multijet estimate. This procedure is performed separately for the RunIIa and RunIIb data, in order to account for potential 
differences due to higher instantaneous luminosity of the RunIIb dataset. Nevertheless, the resulting scale functions are similar, as shown in Fig. 5.5.
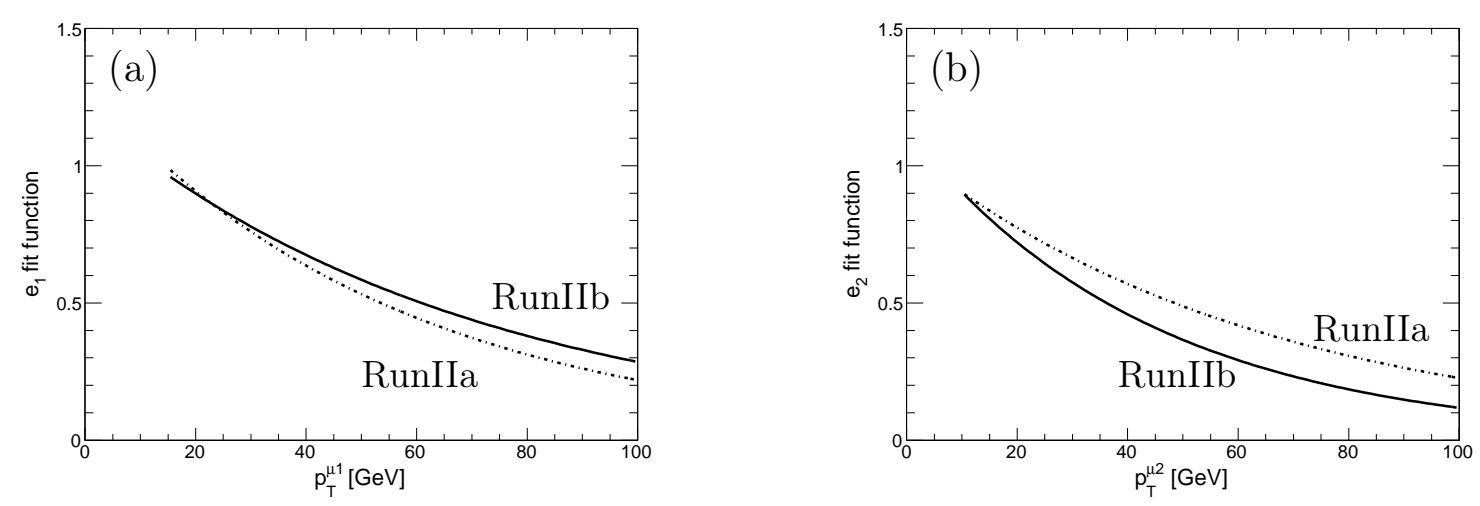

Figure 5.5: Comparison of the scale factors $e_{1}\left(p_{\mathrm{T}}^{\mu_{1}}\right)$ and $e_{2}\left(p_{\mathrm{T}}^{\mu_{2}}\right)$ obtained for the RunIIa and RunIIb epochs. The differences are only visible for high- $p_{\mathrm{T}}$ values, where the fit is dominated by statistical fluctuations.

\subsection{Corrections}

The MC samples do not always reproduce all measured quantities well. This can be attributed to a number of factors. First, the simulation of the hard scattering and of the accompanying QCD phenomena is complicated and difficult, as discussed in section 3.6. Despite the fact that the MC generators usually describe most of the dataset features accurately, for some specific variables, or for a selected subsample of the data, the same might not be true. Second, the detector model implemented in the software is an idealized one. This can lead to a detection efficiency that is overestimated or to a resolution that is better than the effective one.

$\mathrm{D} \varnothing$ collaborators are continuously studying and implementing modifications that aim to improve the accuracy of the simulation. However, it is not possible to re-generate the MC samples each time an improvement is implemented, because this would require significantly more computing resources that those available to the experiment. Thus, corrections are applied to the existing MC samples only. 


\subsubsection{Object Corrections}

The corrections described in this section are specific to each object that is reconstructed in an event. Here, we describe the corrections for muons and jets, which are the main reconstructed objects of this study; similar corrections are applied to electrons and taus, for example.

The identification and reconstruction efficiencies of each muon are also taken into account. These efficiencies are determined with the tag-and-probe method as a function of the muon $\eta_{\mathrm{det}}, p_{\mathrm{T}}$, and isolation; they are provided by the MuonID group and documented in Ref. [39]. In addition, the muon momentum resolution in simulated events is typically $30 \%$ better in $\mathrm{MC}$ than in the actual resolution observed in the data. The origin of this discrepancy is difficult to identify, because it could be attributed to several effects due to limited modelings of several quantities, such as: the single-hit efficiency, the cluster resolution, the detector alignment, and the magnetic field mapping. A consequence of this inconsistency is that the width of the $\mathrm{Z} / \gamma^{*}$ dimuon peak measured in $\mathrm{MC}$ is narrower than that measured in data. To reduce this discrepancy, the $p_{\mathrm{T}}$ of $\mathrm{MC}$ muons is adjusted by a scale factor and smeared. The correction parameters are derived separately for muons with different track quality and from different data-taking epochs, as described in Ref. [66].

The JES and JSSR corrections are applied to jets. In addition, because we require the jets to satisfy the vertex confirmation criterion, a scale factor is applied to MC events to correct the differences in tracking efficiencies between data and MC. This factor, documented by the JetID group in Ref. [67], is computed for each jet in the event as a function of the jet pseudorapidity. For jets with $b$-tag, a taggability and b-tag efficiency correction is applied as well, as documented in Ref. [68; 69].

\subsubsection{Reweightings}

Object corrections are specific to each object that is reconstructed in an event. Sometimes, however, object corrections are not sufficient to obtain a satisfactory modeling of some important variable. In this case, a reweighthing is applied. It modifies the overall event weight. 
It does so by a factor that can be obtained either from a generator variable corresponding to a MC-truth parameter, or from a reconstructed variable. These two types of reweighthings are illustrated in the following two paragraphs.

Generator variable reweighting The reconstructed dilepton- $p_{\mathrm{T}}$, shown in figure 5.6, is a powerful variable to discriminate signal events; therefore, it has to be modeled with great accuracy. The transverse momentum of $\mathrm{Z}$ boson, which is measured in Drell-Yan events as the dilepton $p_{\mathrm{T}}$, is an example of $\mathrm{MC}$ generator variable that is reweighted. According to the calculation performed at leading order in perturbation theory, $\mathrm{W}$ and $\mathrm{Z} / \gamma^{*}$ bosons are produced with small transverse momentum. The leading order prediction corresponds to events in which only two leptons are reconstructed; the dilepton distribution for the 0 - jet subset is shown in figure 5.6(a). However, higher-order effects, such as the emission of gluons and quarks, cause the boson to recoil against jets. As a consequence, the dilepton $p_{\mathrm{T}}$ is, in general, greater for events with jets. The distributions of the dimuon $p_{\mathrm{T}}$ for events with one jet is shown in figure 5.6(b); the distribution for two or more jets is shown in figure 5.6(c). In order to obtain a satisfactory modeling of the dimuon $p_{\mathrm{T}}$ variable, a correction to the $p_{\mathrm{T}}$ of the $\mathrm{Z}$ boson is applied to the ALPGEN-generated $\mathrm{Z} / \gamma^{*}$ samples. The correction is derived from data, after unfolding resolution and acceptance effects, and it is documented in Ref. [70; 71]. A similar correction, documented in Ref. [72], is applied to the $p_{\mathrm{T}}$ of the $\mathrm{W}$ boson for the $\mathrm{W}(+$ jets $/ \gamma)$ samples.
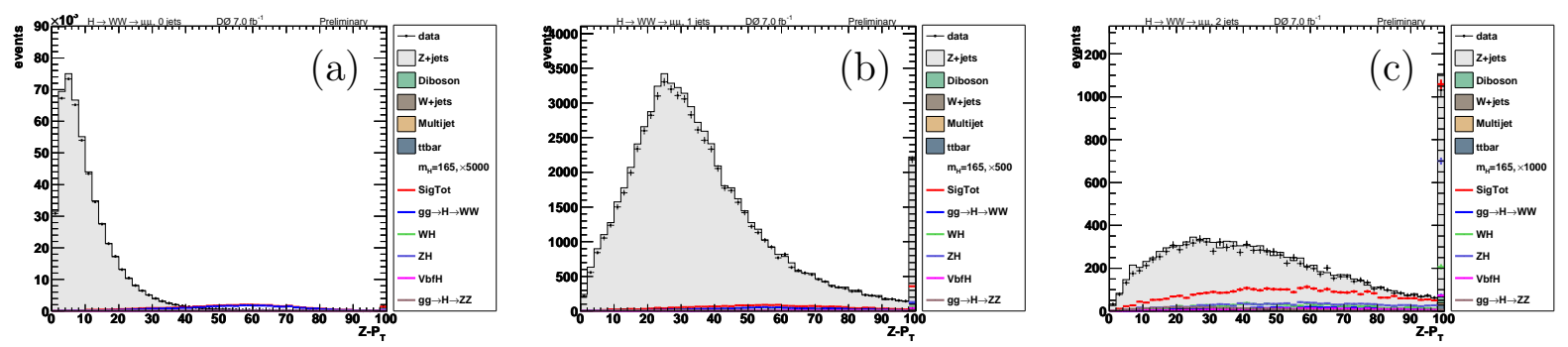

Figure 5.6: Distribution of the dimuon $p_{\mathrm{T}}$ at preselection for the $0-j$ et subset (a), $1-$ jet subset (b), and $2^{+}-$jet subset (c). Here the distributions are shown for the RunIIb sample, after reweighting the $p_{\mathrm{T}}$ of the $\mathrm{W}$ and $\mathrm{Z}$ bosons. The signal normalization is increased to make the signal visible. The last bin contains the overflow events. 
A correction to the PYTHIA-generated diboson WW samples is applied as well-see Ref. [73]. In this case, the momenta of the two bosons are adjusted according to the spectra predicted by the MC@NLO event generator [74], which includes important higher-order QCD corrections.

Reconstructed variable reweighting For some of the variables measured in the event, the correction applied to the event weight is derived from the distribution of the variable itself. The correction factor is computed as the bin-by-bin ratio between data and MC in that distribution. In order not to bias the result, this is done in a sample for which the ratio is not significantly affected by a potential signal. A complete description of the reweightings applied to reconstructed variables is provided in appendix B. The procedure is illustrated with two examples here: the first pertains to the muons' pseudorapidity, and the second the unclustered energy.

The two muons' pseudorapidity distributions predicted by MC do not match the corresponding ones observed in the data. The difference, visible in figure 5.7(a) for $\mu_{1}$, is due to the fact that the trigger efficiency varies for different regions of the detectors. Trigger efficiency corrections are computed for each specific trigger, but because we use an inclusive trigger approach, we cannot apply a single trigger efficiency ${ }^{1}$ correction. Therefore, for each of the two muons, we calculate a reweighting function based on the data/MC ratio of the muon $\eta$ distributions. This function is computed at the preselection stage and, while modifying the event's weight, it preserves the overall normalization of each MC sample. The $\eta$ distribution and reweighting for $\mu_{1}$ are shown in figure 5.7 for RunIIb; this reweighting is calculated separately for RunIIa and RunIIb because the triggers used in the two data-taking periods were different.

The missing transverse energy is one of the variables that discriminate most effectively the

\footnotetext{
${ }^{1}$ A different approach, employed in Ref. [75] for example, consists in applying the lepton-trigger corrections only to those events for which a trigger match can be found for the reconstucted lepton. A reweighting is applied to the other events only. In the context of this thesis, we find that our reweighting procedure leads to a satisfactory data-MC agreement. Nevertheless, a correction similar to that of Ref. [75], based on a combination of trigger corrections and reweightings, is envisioned in the near future.
} 

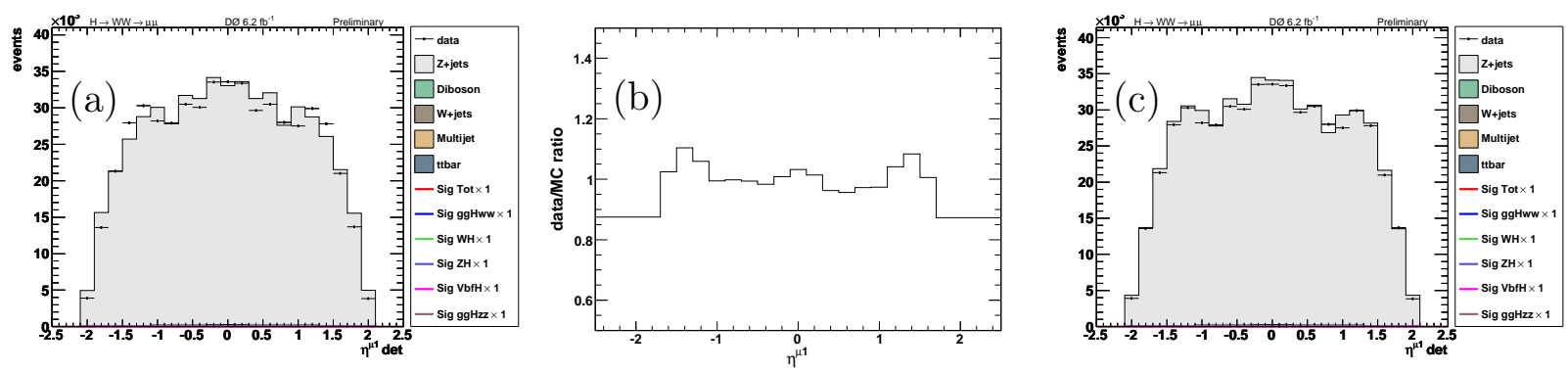

Figure 5.7: The reweighting of the muon $\eta$. The plots, shown here for the RunIIb sample, illustrate the distribution of the variable before (a) and after (c) the reweighting, and the reweighting function being applied (b). This mismodeling is an effect of the detector; thus, the $\eta$ variable used in this reweighting, $\eta_{\text {det }}$, is computed with the origin at the center of the detector, rather than at the PV position.

DY background. Unfortunately, it is also the most difficult one to model correctly, because its determination involves all the other objects reconstructed in the event. In particular, residual data/MC differences in the instantaneous luminosity profile can cause a mismodeling in the $E_{\mathrm{T}}$ distribution. This effect is mostly caused by pile-up jets whose $p_{\mathrm{T}}$ is too low to be recontructed. The energy deposits they cause, however, are not lost: they are included in the unclustered energy (UE). We therefore introduce a reweighting based on the scalar unclustered energy. This reweighting is calculated and applied at preselection. Because it is strictly related to the number of jets recontructed in the event, the data/MC ratio histograms are computed separately for the subsamples with zero, one, two or more jets. The effects of the UE reweighting are shown in figure 5.8.

\subsection{Multivariate Discriminant}

In most analyses, several variables can be used to discriminate signal events from background ones. A traditional approach is to reject most of the background events by applying cuts on all but one variables. The distribution of the last variable is then used to compute the statistical significance of the measurement. However, this approach does not use the information that is available in the correlations among variables.

Multivariate classifiers can exploit such correlations and increase the sensitivity of the 

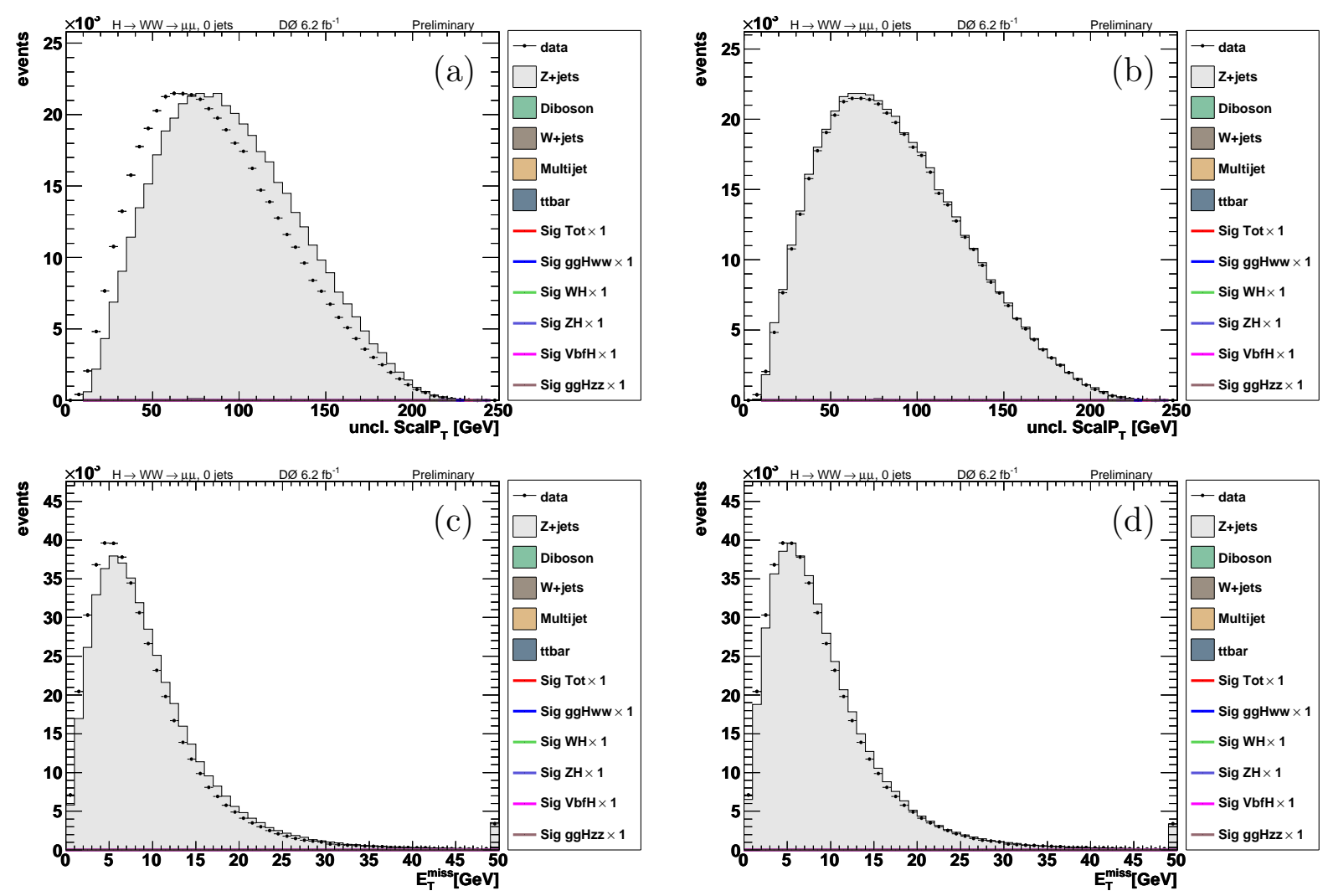

Figure 5.8: Effects of the scalar unclustered energy reweighting shown at the preselection stage for the RunIIb subset with zero recontructed jets. The distribution of the scalar unclustered energy before (a) and after (b) the reweighting; the distribution of the missing transverse energy before (c) and after (d) the reweighting. 
analysis. While several implementations of different classifiers and learning algorithms are available, in general, the mode of operation is the same. A training set, which comprise the input variables and the desired output for each event, is used to optimize the parameters of the classifier. The classifier's output is then used to separate the signal from the backgrounds and to build a distribution that can be used to test the hypothesis under study.

In this search, we use two types of multivariate discriminants: neural networks and decision trees. In both cases, we use a two-step procedure: the first step aims at reducing the large number of backgrounds events due to the $\mathrm{Z} / \gamma^{*}(+$ jets $/ \gamma)$ process; the second step consists in building a final discriminant that can be used to separate the signal candidate events from the remaining background ones.

Neural networks are used in the $5.4 \mathrm{fb}^{-1}$ analysis. In the NN study, the $\mathrm{Z} / \gamma^{*}(+$ jets $/ \gamma)$ background is rejected with cut-based criteria and the neural networks as a final discriminant.

Decision trees are used in the $8.1 \mathrm{fb}^{-1}$ analysis. A first discriminant, which we call the Drell-Yan decision tree (DY DT), is trained against the $\mathrm{Z} / \gamma^{*}(+$ jets $/ \gamma)$ background. We then select events for which the DY DT output is above some threshold, and we train a second decision tree to separate the signal from all backgrounds. We call this second discriminant the final discriminant decision tree (FD DT).

\subsubsection{Event Variables}

Several variables are used in both studies - the $5.4 \mathrm{fb}^{-1} \mathrm{NN}$ analysis and the $8.1 \mathrm{fb}^{-1} \mathrm{DT}$ analysis. In the simplest case, these variables are required to be above a minimum value or below a maximum value; otherwise, these variables are used as the inputs to a multivariate discriminant. In section 5.4.1, we illustrate all of the variables that are relevant to this search; in sections 5.5 and 5.6 we describe the use of these variables within the subsequent steps of the two studies. 


\section{Lepton quality variables}

In addition to the official muon-quality criteria, we use two muon-quality variables to reject background events. The first variable is the muon isolation. It can suppress the multijet and the $\mathrm{W}(+$ jets $/ \gamma)$ backgrounds, for which we expect at least one of the muons to be a misrecontructed jet. We use either the track isolation, or a combination of the track and calorimeter isolations. The second variable is the muon quality. Candidate signal events are more likely to be true if the muons are well-reconstructed. We define a discrete muonquality variable based on the muon reconstruction categories described in section 3.3.2. The muon-quality is defined in equation (5.3), and we use the minimum quality of the two muons, $\min _{\mu_{1}, \mu_{2}}(\mu-$ qual), as an input to the multivariate discriminants.

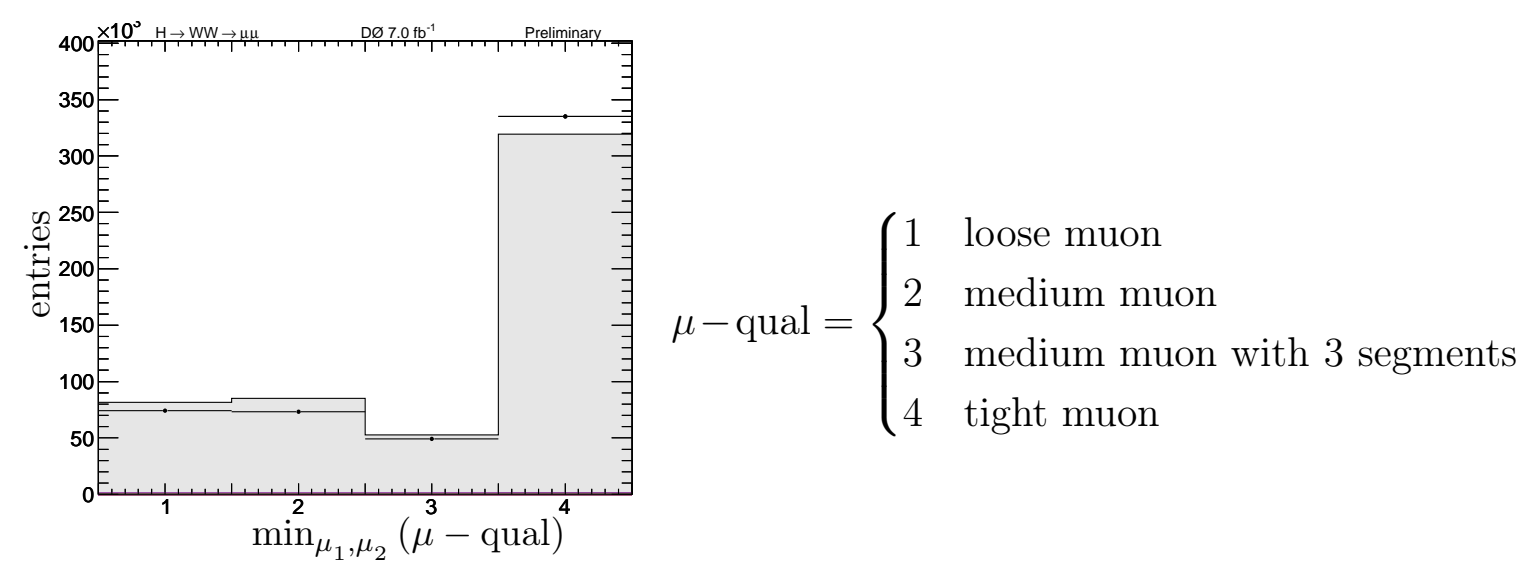

\section{Lepton kinematic variables}

Most of the time, the variables describing the kinematic of the leptons reconstructed in the event provide the best discrimination between signal and background. In the simplest case of a $\mathrm{H} \rightarrow \mathrm{W}^{+} \mathrm{W}^{-} \rightarrow \bar{\ell} \nu \ell \bar{\nu}$ event, the Higgs boson is produced at rest; the energy from its decay is split almost evenly among the two Ws; it is then split again almost evenly between the muons and the neutrinos. Thus, the $p_{\mathrm{T}}^{\mu_{1}}$ and $p_{\mathrm{T}}^{\mu_{2}}$ variables constitute good indicators of the nature of an event, as shown in figure 5.13.

The dimuon invariant mass $M_{\mu \mu}$ is shown in figure 5.2. It is the most intuitive choice 

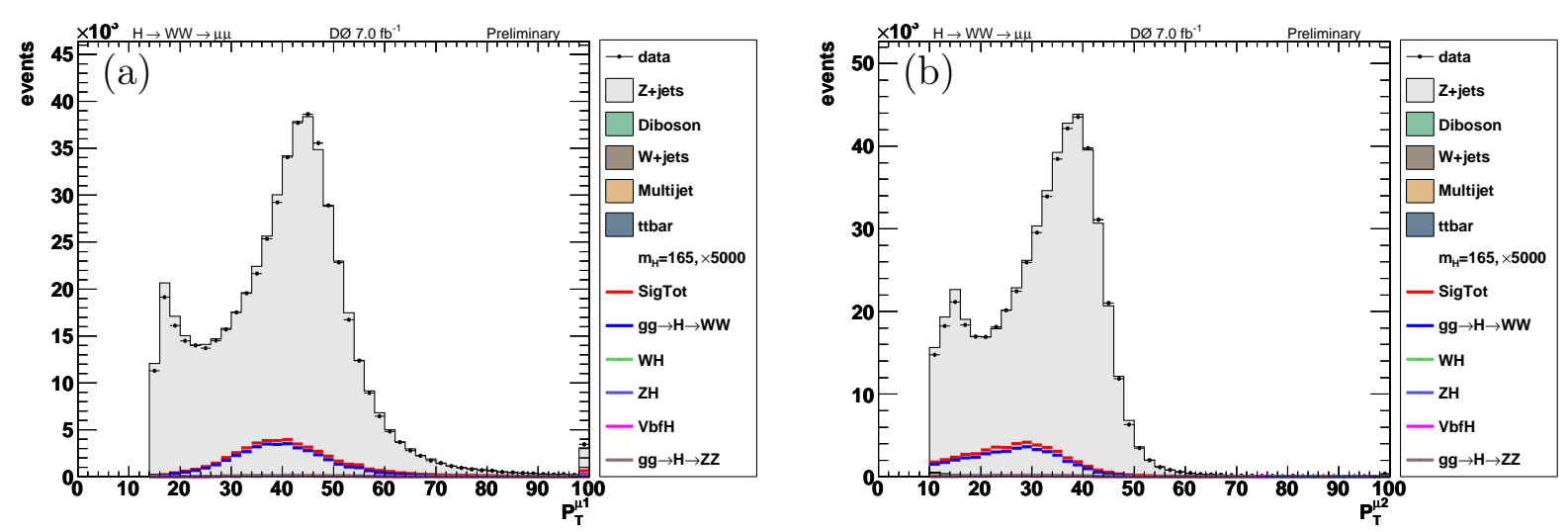

Figure 5.9: Transverse momentum distributions of $\mu_{1}$ (a), and $\mu_{2}$ (b), for the RunIIb dataset at preselection. The expected signal spectrum is shown for $m_{\mathrm{H}}=165 \mathrm{GeV}$, with the normalization increased 5000 times.

for a variable to reject $\mathrm{Z} / \gamma^{*}(+$ jets $/ \gamma)$ events, because most of these events are in the $\mathrm{Z}$ peak at $91 \mathrm{GeV}$. Shown in figure 5.6 is the dimuon total transverse momentum $p_{\mathrm{T}}^{\mu \mu}$ that, along with the opening angle between the two muons, can provide good discrimination against DY events. For most DY events, the two muons have approximately the same momentum and are diametrically opposed in the transverse plane. As a consequence, the dimuon system has little total momentum. Conversely, for $\mathrm{Z} / \gamma^{*}(+$ jets $/ \gamma)$ events in which the $\mathrm{Z}$ boson recoils against one or more jets, the muons are not diametrically opposed and have a high $p_{\mathrm{T}}^{\mu \mu}$.

The opening angle between the two muons, measured either as $\Delta R$ or as $\Delta \phi$ and shown in figure 5.10, is expected to be small for signal events. Because the Higgs boson has spin equal to zero, and the $\mathrm{W}$ bosons have spin equal to one, angular momentum conservation requires that the Ws' spins be anti-correlated. Due to the parity-violating nature of the weak force, the left-handed $\mu^{-}$is emitted along the $\mathrm{W}^{-}$spin direction, and the right-handed $\mu^{+}$is emitted along the $\mathrm{W}^{+}$spin direction. For this reason, the two muons generated in a $H \rightarrow W^{+} W^{-}$ decay tend to be collinear. Nevertheless, the discriminating power of the opening angle is reduced in the hypothesis of a heavy Higgs boson, namely with $\mathrm{m}_{\mathrm{H}}>180 \mathrm{GeV}$; in this case, the two $\mathrm{W}$ bosons are boosted, leading to more collimated products from the $\mathrm{W} \rightarrow \ell \nu$ decay.

The direction in $\eta$ of the two muons encodes useful information as well, in particular for 

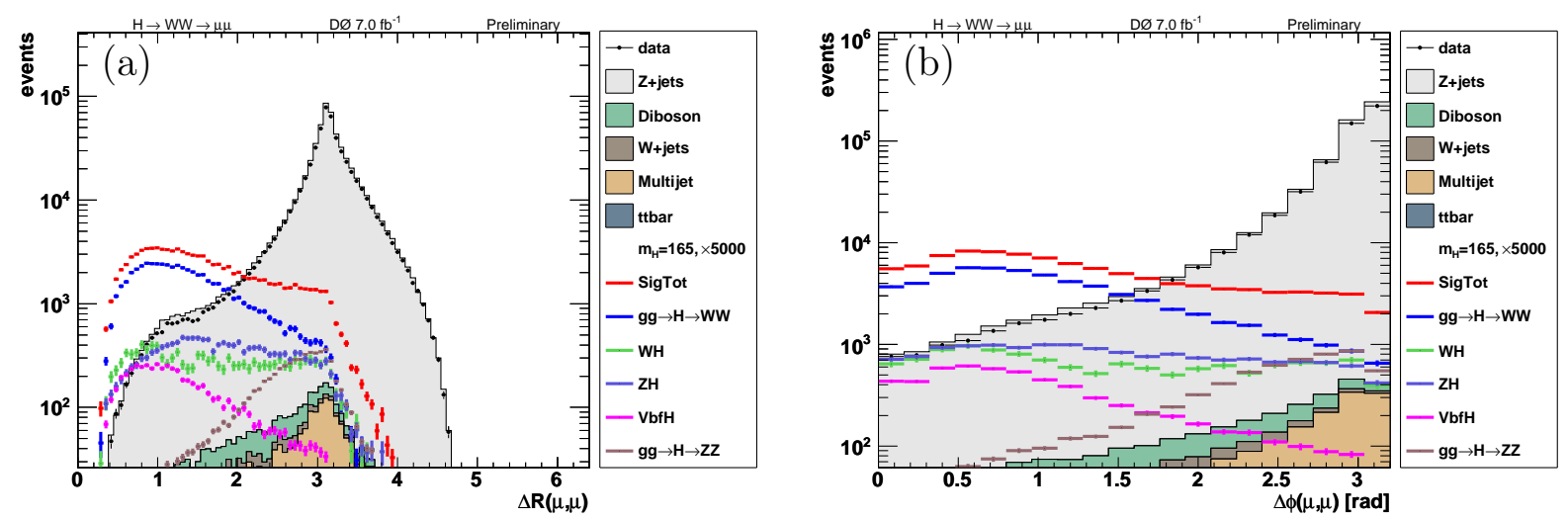

Figure 5.10: The opening angle between the two muons: (a) measured in $(\eta, \phi)$ as $\Delta \mathrm{R}(\mu, \mu)$, and (b) measured in the transverse plane as $\Delta \phi(\mu, \mu)$. In these distributions corresponding to the RunIIb dataset at preselection, the signal spectrum is shown for $\mathrm{m}_{\mathrm{H}}=165 \mathrm{GeV}$, with normalization increased 5000 times.

events due to the $\mathrm{W}(+$ jets $/ \gamma)$ background in which a $\mathrm{W}$ boson is produced. On account of the asymmetry in the quarks' PDF for protons and antiprotons, the $\mathrm{W}^{+}$boson tends to be produced along the direction of the proton beam, with positive $\eta$, whereas the $\mathrm{W}^{-}$ boson tends to be produced along the antiproton direction. For this reason, the variables $\operatorname{sign}\left(q_{1}\right) \cdot \eta_{1}$ and $\operatorname{sign}\left(q_{2}\right) \cdot \eta_{2}$ can help discriminate from the $\mathrm{W}(+$ jets $/ \gamma)$ background. For this background, most of the time, $\mu_{1}$ is due to the $\mathrm{W}^{ \pm}$decay; $\mu_{2}$, with lower $p_{\mathrm{T}}$, is due to a misreconstructed jet. The $\operatorname{sign}\left(q_{1}\right) \cdot \eta_{1}$ distribution for the RunIIb dataset at final selection is shown in figure 5.11 .

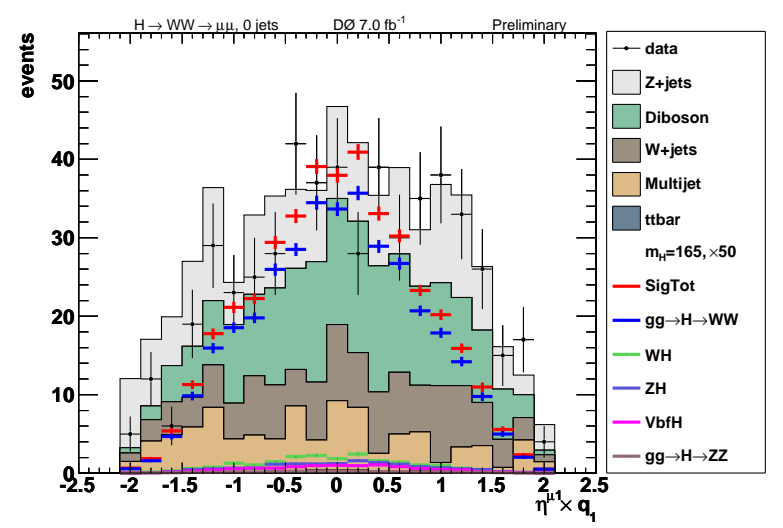

Figure 5.11: The $\operatorname{sign}\left(q_{1}\right) \cdot \eta_{1}$ distribution for the RunIIb dataset at final selection. The asymmetry due to the $\mathrm{W}^{ \pm}$production is visible for the $\mathrm{W}(+$ jets $/ \gamma)$ background; this asymmetry is not visible at preselection, where most of the events are due to $\mathrm{Z} / \gamma^{*}(+$ jets $/ \gamma)$. 


\section{Jet variables}

The main signal process, $\mathrm{gg} \rightarrow \mathrm{H} \rightarrow \mathrm{W}^{+} \mathrm{W}^{-} \rightarrow \bar{\ell} \nu \ell \bar{\nu}$, is expected to have low jet-activity, as shown in figure 5.12. The number of jets reconstructed in the event is a useful quantity to reject backgrounds, such as $\mathrm{W}(+$ jets $/ \gamma)$ and $t \bar{t}$. We consider jets that satisfy the vertex confirmation requirement, and that have a $p_{\mathrm{T}}>20 \mathrm{GeV}$. When there are such jets, several reconstructed quantities, beside $N_{\text {jets }}$, can help identify potential signal events.

The jet energy, and the jet $(\eta, \phi)$ coordinates provide useful information. In addition, for $\mathrm{Z} / \gamma^{*}(+$ jets $/ \gamma)$ events, the jet usually recoils against the dilepton system. The jet separation from other objects assesses their quality: a lepton that is well separed from a jet is unlikely to be due to a hadron decay within the jet, and a $E_{\mathrm{T}}$ that is opposite in $\phi$ to a jet is likely to be due to a mismeasurement of the jet energy.

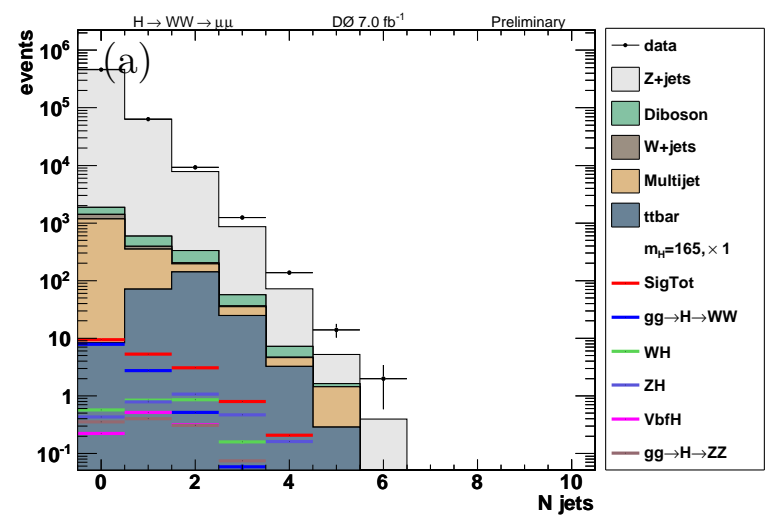

Figure 5.12: Number of jets reconstructed in each event.

In events with two jets, the dijet invariant mass helps identify jet pairs from massive vector boson decays. The reconstruction of $\mathrm{W} \rightarrow j j$ or $\mathrm{Z} \rightarrow j j$ decays is a signature that can be used to find potential VH events.

Lastly, the $b$-tag output of each jet is useful to the identification of t $\bar{t}$ background events, in which two $b$-jets should be reconstructed from $\mathrm{t} \rightarrow \mathrm{Wb}$. 

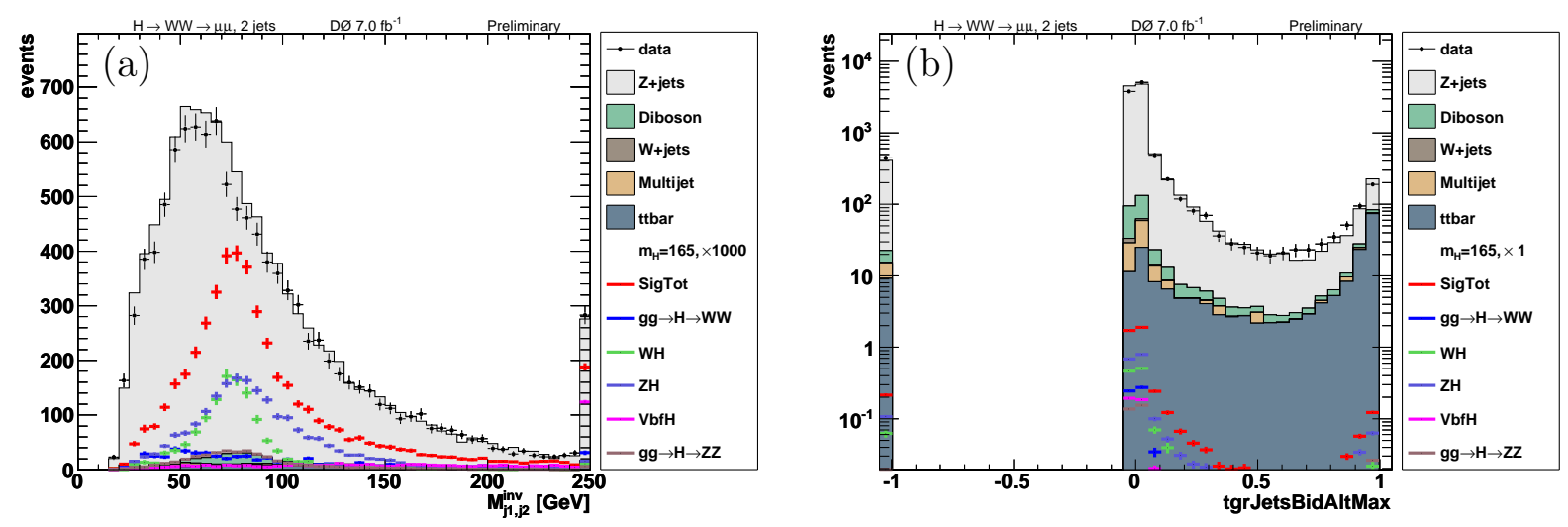

Figure 5.13: Two discriminating variables for events with two or more jets, shown here for the RunIIb dataset and for $\mathrm{m}_{\mathrm{H}}=165 \mathrm{GeV}$. The dijet invariant mass (a): the most numerous signal contributions are those due to associated production, for which $M_{j j}$ has a peak at $M_{\mathrm{W}}$ or $M_{\mathrm{Z}}$. The maximum value of the $b$-tag output computed for all taggable jets (b). Non-taggable jets correspond to the most negative bin.

\section{Missing-energy variables}

Signal events are expected to have significant $E_{\mathrm{T}}$, as shown in figure 5.14(a). In addition to the genuine contributions from neutrinos, however, the transverse energy imbalance can result from poorly measured momenta or energies. The extent of these fictitious contributions can be estimated from the angular separation of muons and jets from the $E_{\mathrm{T}}$ direction. For example, the angular separation $\Delta \phi\left(E_{\mathrm{T}}, \mu_{1}\right)$ is shown in figure 5.14(b). More advanced algorithms, described in the following paragraphs, can better detect real $E_{\mathrm{T}}$.

The scaled missing transverse energy, $E_{\mathrm{T}}^{\text {scaled }}$, aims to assess the $E_{\mathrm{T}}$ significance associated with jets. Its computation relies on the $\eta$-dependent parametrization of the jet energy resolution, which allows for an estimate of each jet's energy fluctuation $\Delta E^{\text {jet }}(\eta)$, as documented in Ref. [76]. Contributions due to any jet reconstructed in the event are taken into account; these include jets that are not from the primary vertex. The scaled missing transverse energy is then defined as $E_{\mathrm{T}}$ divided by the sum in quadrature of all possible fluctuations in the jets' energy measurements:

$$
E_{\mathrm{T}}^{\text {scaled }}=\frac{E_{\mathrm{T}}}{\sqrt{\sum_{\text {jets }}\left(\Delta E^{\text {jet }} \cdot \sin \theta^{\text {jet }} \cdot \cos \Delta \phi\left(\text { jet }, E_{\mathrm{T}}\right)\right)^{2}}}
$$



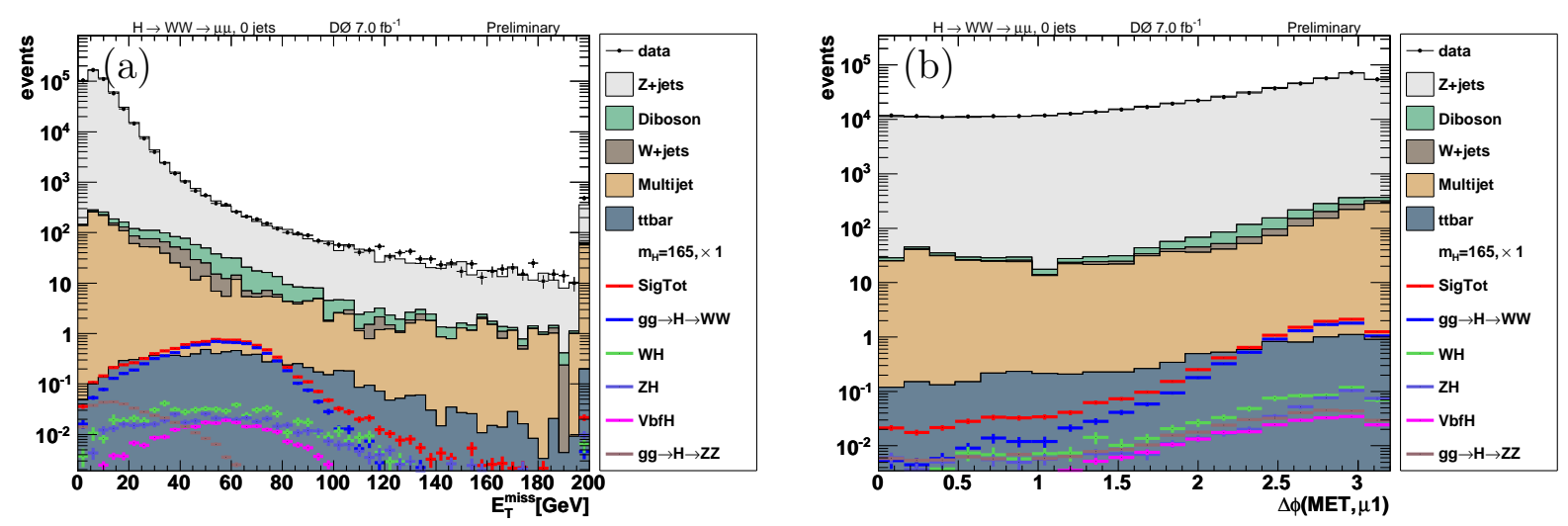

Figure 5.14: Clean events without reconstructed jets: (a) missing energy distribution, shown here for the RunIIb dataset. For $\mathrm{m}_{\mathrm{H}}=165 \mathrm{GeV}$ a missing energy of about $70 \mathrm{GeV}$ is expected. For a large fraction of events, the missing energy is in the opposite azimuthal direction of the muon with highest $p_{\mathrm{T}}$, indicating that the $E_{\mathrm{T}}$ could be in fact due to a fluctuation in the $p_{\mathrm{T}}$ measurement.

The $E_{\mathrm{T}}^{\text {scaled }}$ distribution is shown in figure 5.15(a) at preselection stage for events with at least one jet.

The special missing transverse energy, $E_{\mathrm{T}}^{\text {special }}$, is defined to reduce the impact on $E_{\mathrm{T}}$ of any potential mismeasurement, from either jets or leptons. It proves particularly useful to reject $\mathrm{Z} / \gamma^{*}(+$ jets $/ \gamma)$ events, and is defined as follows:

$$
E_{\mathrm{T}}^{\text {special }}= \begin{cases}E_{\mathrm{T}} & \text { if } \Delta \phi\left(E_{\mathrm{T}}, \ell \| j\right)>\pi / 2 \\ E_{\mathrm{T}} \cdot \sin \Delta \phi\left(E_{\mathrm{T}}, \ell \| j\right) & \text { otherwise }\end{cases}
$$

where $\Delta \phi\left(E_{\mathrm{T}}, \ell \| j\right)$ is the opening angle in the transverse plane between the $E_{\mathrm{T}}$ and the nearest lepton or jet. The $\mathcal{E}_{\mathrm{T}}^{\text {special }}$ distribution is shown in figure 5.15(b) at the preselection stage.

The complete event kinematic cannot be reconstructed because the 4-momenta of the two neutrinos cannot be disentangled. Nevertheless, the $E_{\mathrm{T}} 4$-momentum can be combined with the lepton 4-momenta, and provides indirect information about the invariant mass of the WW system.

The transverse $\mathrm{W}$ mass is defined as $M_{\mathrm{T}}\left(\ell, E_{\mathrm{T}}\right)=\sqrt{2 p_{\mathrm{T}}^{\ell} E_{\mathrm{T}}\left(1-\cos \Delta \phi\left(\ell, E_{\mathrm{T}}\right)\right)}$. The 

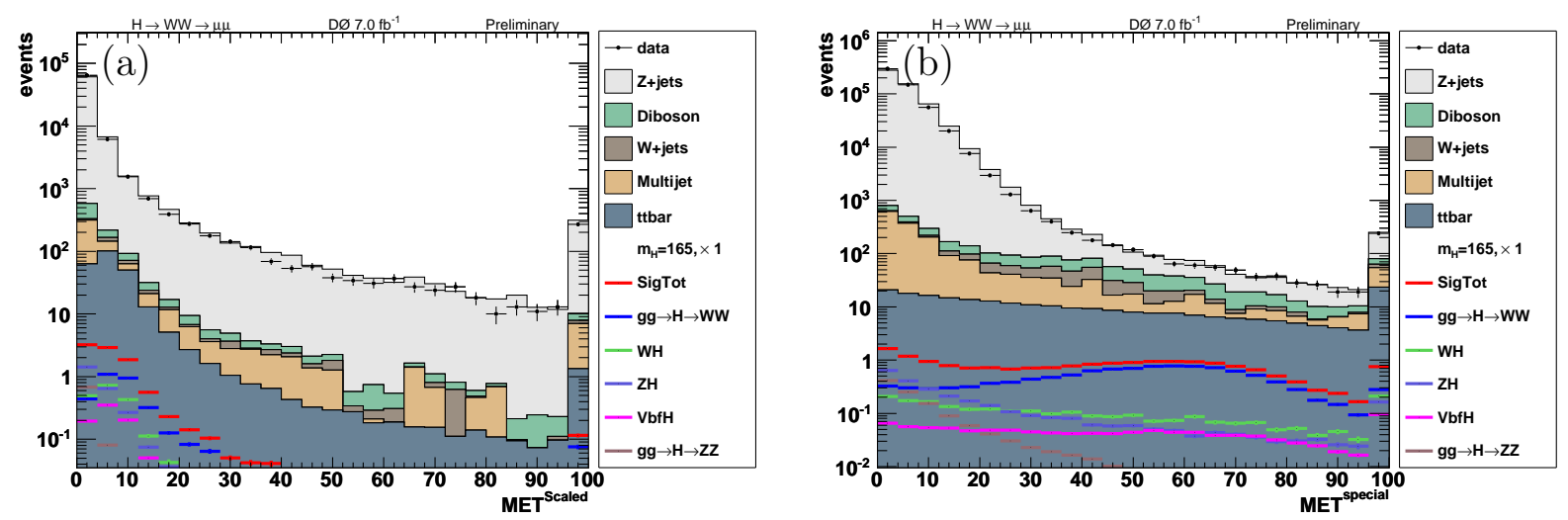

Figure 5.15: (a) The $E_{\mathrm{T}}^{\text {scaled }}$ distribution at preselection, for RunIIb events with at least one reconstructed jet. (b) The $E_{\mathrm{T}}^{\text {special }}$ at preselection, for RunIIb events. Signal histograms are shown for $\mathrm{m}_{\mathrm{H}}=165 \mathrm{GeV}$.

transverse $\mathrm{W}$ mass, not to be confused with the kinematic transverse mass, was introduced when the $\mathrm{W}$ boson was first observed [77]. When a single $\mathrm{W}$ is produced, $M_{\mathrm{T}}$ is constrained to be less than $\mathrm{m}_{\mathrm{W}}$, and provides a way to estimate the $\mathrm{W}$ mass from the jacobian peak in the $M_{\mathrm{T}}$ distribution. For this project, we compute the values of the transverse mass for the two leptons, and then use their minimum $M_{\mathrm{T}}^{\min }=\min \left[M_{\mathrm{T}}\left(\ell_{1}, E_{\mathrm{T}}\right), M_{\mathrm{T}}\left(\ell_{2}, E_{\mathrm{T}}\right)\right]$, which can provide indirect information on the mass of the Higgs boson; the $M_{\mathrm{T}}^{\min }$ distribution is shown in figure 5.16(a).

The stransverse mass $M_{\mathrm{T} 2}$, shown in figure 5.16(b), is an extension of the $M_{\mathrm{T}}$ variable to the case in which there are multiple decay products that cannot be detected. It was first proposed by Summers and Lesters [78] in the context of the searches for supersymmetric decay chains. Strictly speaking, $M_{\mathrm{T} 2}$ is not defined as an event variable, but rather as a function of the two unknown neutrino momenta. For each event, this function is then minimized and, in fact, we refer to $M_{\mathrm{T} 2}$ to indicate its minimum value. The minimization is performed over all possible 2-momenta $\boldsymbol{p}_{1}$ and $\boldsymbol{p}_{2}$ whose sum matches the measured missing transverse energy $\boldsymbol{p}_{T}=\left(E_{x}, E_{y}\right)$ :

$$
M_{\mathrm{T} 2}^{2}=\min _{\boldsymbol{p}_{1}+\boldsymbol{p}_{2}=\boldsymbol{p}_{T}}\left[\max \left\{M_{\mathrm{T}}^{2}\left(\ell^{+}, \nu_{1}\right), M_{\mathrm{T}}^{2}\left(\ell^{-}, \nu_{2}\right)\right\}\right] .
$$

To perform the minimization and compute $M_{\mathrm{T} 2}$, we use Cheng and Han's implementation [79]. 
This implementation is faster than the algorithms available in other libraries, and it requires minimal constraints, namely the mass-shell conditions for the Ws and the constraint on the total missing momentum measured.
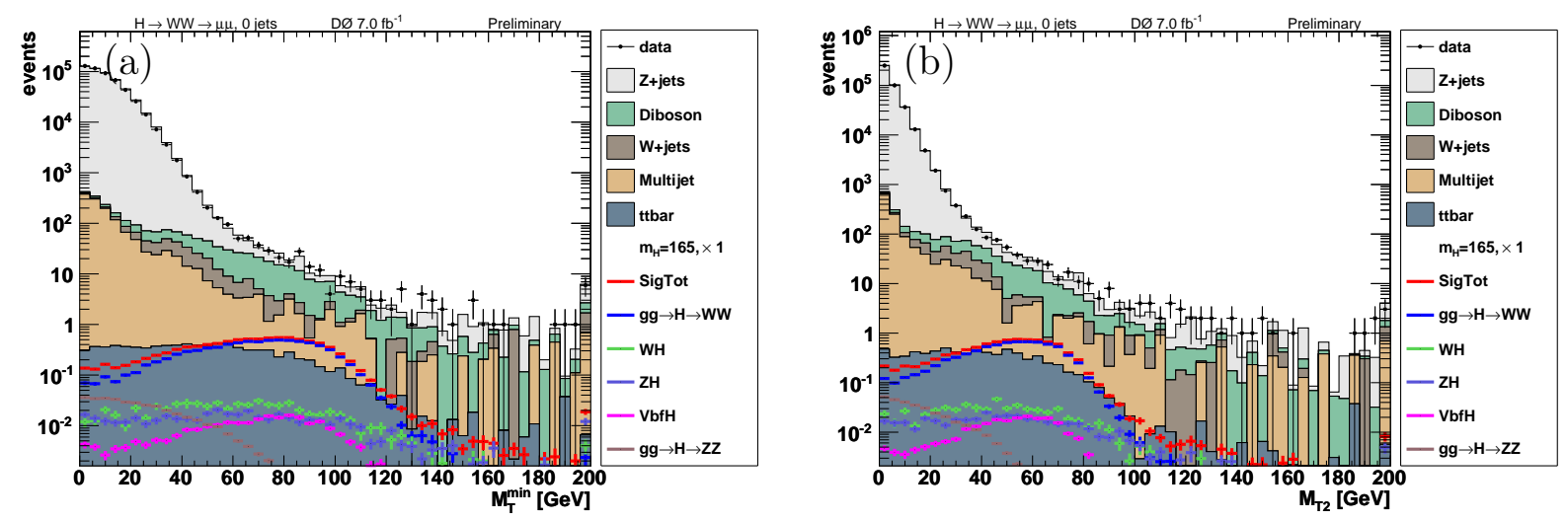

Figure 5.16: The minimum transverse mass (a), and the stransverse mass (b). These two variables have the best discriminating power when there are no jets in the events, all of the event's kinematic information is in the four-momenta of the two leptons and of the missing energy. Here, they are shown for the RunIIb dataset events with no jets.

\subsection{DY Rejection}

Two different strategies, illustrated in sections 5.5.1 and 5.5.2, are used to reject the $\mathrm{Z} / \gamma^{*}(+$ jets $/ \gamma)$ background. The first one, used in the $5.4 \mathrm{fb}^{-1}$ analysis, relies on cut-based criteria; the second one, used in the $8.1 \mathrm{fb}^{-1}$ analysis, relies on a multivariate discriminant.

\subsubsection{Cut-based DY Rejection}

In the $\mathrm{NN} 5.4 \mathrm{fb}^{-1}$ analysis, the $\mathrm{Z} / \gamma^{*}(+$ jets $/ \gamma)$ background is suppressed with three cut-based criteria, as illustrated in table 5.2. These selection criteria are optimized for a Higgs boson with a mass of $165 \mathrm{GeV}$. This mass value corresponds to the hypothesis in which this analysis has its maximum sensitivity. For $\mathrm{m}_{\mathrm{H}}=165 \mathrm{GeV}, 99.6 \%$ of the $\mathrm{Z} / \gamma^{*}$ events are suppressed with these criteria; at the same time, less than $30 \%$ of the signal events are lost. The number of events selected with this cut-based criteria is reported in table 5.3. 


\begin{tabular}{lllr}
\hline Variable description & \multicolumn{2}{c}{ Criterion } \\
\hline Opening azimuthal angle & $\Delta \phi\left(\mu_{1}, \mu_{2}\right)$ & $<2.0 \mathrm{rad}$ \\
Missing transverse energy & $E_{\mathrm{T}}$ & $>25.0 \mathrm{GeV}$ \\
Minimum transverse mass & $M_{\mathrm{T}}^{\min }$ & $>20.0 \mathrm{GeV}$ \\
\hline
\end{tabular}

Table 5.2: Selection criteria for the $5.4 \mathrm{fb}^{-1}$ analysis.

\begin{tabular}{lcc}
\hline & \multicolumn{2}{c}{$\mu^{+} \mu^{-}$} \\
Sample & Preselection & Final selection \\
\hline$Z / \gamma^{*} \rightarrow \mu^{+} \mu^{-}$ & 373582. & $1247 \pm 37$ \\
$Z / \gamma^{*} \rightarrow \tau^{+} \tau^{-}$ & 2659. & $12.0 \pm 0.7$ \\
$\mathrm{t} \overline{\mathrm{t}}$ & 184. & $74.6 \pm 6.8$ \\
$\mathrm{~W}(+$ jets $/ \gamma)$ & 236. & $91.5 \pm 6.5$ \\
$W W$ & 272. & $107 \pm 9$ \\
$W Z$ & 171. & $21.5 \pm 2.0$ \\
$Z Z$ & 147. & $18.0 \pm 1.8$ \\
Multijet & 408. & $53.8 \pm 10.3$ \\
Signal, $\mathrm{m}_{\mathrm{H}}=165 \mathrm{GeV}$ & 12.7 & $9.0 \pm 1.0$ \\
Total background & 377659. & $1625 \pm 41$ \\
\hline Data & 384083 & 1613 \\
\hline
\end{tabular}

Table 5.3: Expected and observed event yields for the $5.4 \mathrm{fb}^{-1}$ dataset, after preselection and at the final selection. The systematic uncertainty after fitting is shown for all samples at the final selection stage.

\subsubsection{Decision Tree DY Rejection}

While the cut-based criteria are quite effective in the $\mathrm{m}_{\mathrm{H}}=165 \mathrm{GeV}$ region, where $\mathrm{gg} \rightarrow$ $\mathrm{H} \rightarrow \mathrm{W}^{+} \mathrm{W}^{-} \rightarrow \bar{\ell} \nu \ell \bar{\nu}$ is the main signal contribution, their effectiveness is reduced outside of this mass region. For mass values approaching to $130 \mathrm{GeV}$ or to $200 \mathrm{GeV}$, signal production processes other than $\mathrm{gg} \rightarrow \mathrm{H}$ provide significant contributions, and these processes do not have the clear signature that $\mathrm{gg} \rightarrow \mathrm{H} \rightarrow \mathrm{W}^{+} \mathrm{W}^{-} \rightarrow \bar{\ell} \nu \ell \bar{\nu}$ has. At low $\mathrm{m}_{\mathrm{H}}$, many signal events are due to the associated production, and VH events often have jets from the decay of the weak boson. At high $\mathrm{m}_{\mathrm{H}}$, the branching ratio for $H \rightarrow \mathrm{ZZ}$ becomes significant. For all of the possible $H \rightarrow \mathrm{ZZ}$ decays, $\mathrm{ZZ} \rightarrow \ell \bar{\ell} \ell \bar{\ell}, \mathrm{ZZ} \rightarrow \ell \bar{\ell} \nu \bar{\nu}$, and $\mathrm{ZZ} \rightarrow \ell \bar{\ell} j j$, the event's kinematics are different from the $H \rightarrow W^{+} W^{-}$ones that are typical at $\mathrm{m}_{\mathrm{H}} \simeq 165 \mathrm{GeV}$. If the $\mathrm{ZZ} \rightarrow \ell \bar{\ell} \ell \bar{\ell}$ 
decay takes place, the apparent missing energy must originate from leptons that go through a low-efficiency region of the detector and are therefore not reconstructed. If the $\mathrm{ZZ} \rightarrow \ell \bar{\ell} \nu \bar{\nu}$ decay takes place, the two leptons do not display the angular correlations that are typical of $H \rightarrow W^{+} W^{-}$. If the $\mathrm{ZZ} \rightarrow \ell \bar{\ell} j j$ decay takes place, the missing energy is mostly due to jet mismeasurements, making signal events look much like $\mathrm{Z} / \gamma^{*}(+$ jets $/ \gamma)$ ones.

In order to exploit the distinct signal contributions that are present in different $\mathrm{m}_{\mathrm{H}}$ regions, and to avoid the signal loss caused by the cut-based criteria, we use a different approach in the most recent analysis of the $8.1 \mathrm{fb}^{-1}$ dataset. In the $8.1 \mathrm{fb}^{-1}$ analysis, we employ decision trees rather than kinematic criteria to reduce the number of $\mathrm{Z} / \gamma^{*}(+$ jets $/ \gamma)$ events.

A DT discriminant is trained at each $\mathrm{m}_{\mathrm{H}}$ using only the DY and the signal samples. The $\mathrm{Z} / \gamma^{*}(+$ jets $/ \gamma)$ background is then suppressed by requiring a minimum value of the DT output. We use the decision trees implemented in the TMVA [80] software package. Each DT discriminant is composed of two hundred trees in a random forest configuration. The exact parameters of the DT discriminant are listed in table 5.4. The configuration of the

\begin{tabular}{lr}
\hline \multicolumn{1}{c}{ Random Forest DT configuration } & Value \\
Parameter & 200 \\
\hline Number of trees & 8 \\
Number of variables used per node splitting & 9 \\
Maximal depth of the tree & 50 \\
Minimum events per final leaf & False \\
UseYesNoLeaf flag & True \\
UseRandomisedTrees flag & Gini index \\
SeparationType & False \\
Pruning &
\end{tabular}

Table 5.4: Main parameters of the RF discriminant used for the DY DT and FD DT. An extensive description of the paramters can be found in the TMVA documentation [80].

DY DT is the same one used for the FD DT. Nevertheless, the input variables used for the Drell-Yan discriminant are only a subset of those used for final discriminant, as indicated in table 5.5. Decision trees are trained separately for each jet multiplicity; this allows for the inclusion of the additional pieces of information from one or more jets recontructed in 
the event. The DY DT output distributions are shown in figure 5.17 for $\mathrm{m}_{\mathrm{H}}=165 \mathrm{GeV}$, for events with zero, one, two or more jets.
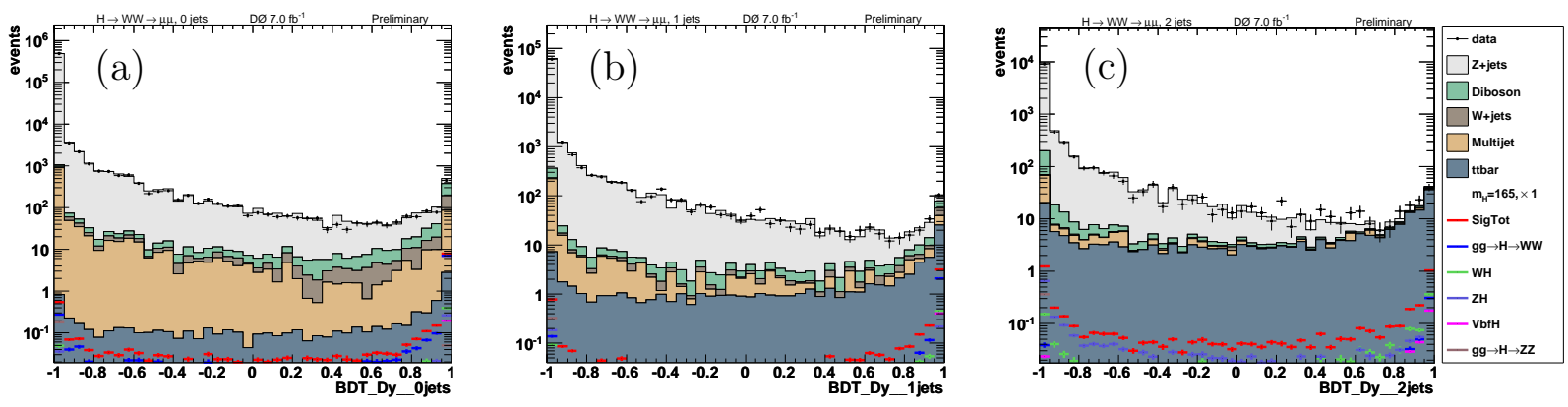

Figure 5.17: The DY DT distributions for events with no jets (a), with one jet (b), and with two or more jets (c).

\begin{tabular}{|c|c|c|}
\hline & Decision T & \\
\hline & DY or FD & FD \\
\hline Any $N_{\text {jets }}$ & $\begin{array}{l}p_{\mathrm{T} \ell_{1}}, p_{\mathrm{T} \ell_{2}}, p_{\mathrm{T} \ell \ell} \\
\Delta \phi(\ell, \ell), \Delta \mathrm{R}(\ell, \ell), q_{1} \cdot \eta_{1}, q_{2} \cdot \eta_{2} \\
E_{\mathrm{T}}, E_{\mathrm{T}}^{\text {ssaled }}, E_{\mathrm{T}}^{\text {special }} \\
M_{\mathrm{T}}^{\text {min }}, M_{\mathrm{T} 2}, M_{\mathrm{T}}\left(\ell \ell, E_{\mathrm{T}}\right) \\
\min _{\ell_{1}, \ell_{2}} \Delta \phi\left(E_{\mathrm{T}}, \ell\right), \max _{\ell_{1}, \ell_{2}} \Delta \phi\left(E_{\mathrm{T}}, \ell\right)\end{array}$ & $\begin{array}{l}\min _{\mu_{1}, \mu_{2}}[\mu-\text { qual }] \\
\text { trackIso }_{\text {scaled }} \text { for } \mu_{1}, \mu_{2}\end{array}$ \\
\hline$N_{\text {jets }}>0$ & $\begin{array}{l}p_{\mathrm{T} j_{1}} \\
\Delta \phi\left(\not E_{\mathrm{T}}, j_{1}\right)\end{array}$ & $b-\operatorname{tag} j_{1}$ \\
\hline$N_{\text {jets }}>1$ & $\begin{array}{l}p_{\mathrm{T} j_{2}} \\
\Delta \eta\left(j_{1}, j_{2}\right), M\left(j_{1} j_{2}\right), M\left(j_{1} j_{2} \ell \ell\right) \\
\min _{j_{1}, j_{2}} \Delta \phi\left(E_{\mathrm{T}}, j\right), \max _{j_{1}, j_{2}} \Delta \phi\left(E_{\mathrm{T}}, j\right)\end{array}$ & $b-\operatorname{tag} j_{2}$ \\
\hline
\end{tabular}

Table 5.5: Input variables for the DY DT and for the FD DT. The core set of variables is used for the 0-jet DY DT. In addition, jet-related variables are used when one or more jets are reconstructed in the event. Additional variables, such as lepton isolation and jet $b-\operatorname{tag}$, are used only for the FD DT.

We then only select the events for which the DY DT output is above a certain threshold. The threshold is chosen so that we obtain at least the same DY rejection that would be obtained with the cut-based criteria, as illustrated in figure 5.18. The value of the threshold is chosen for each DY DT training, or equivalently for each jet multiplicity and for each $\mathrm{m}_{\mathrm{H}}$. The number of events selected with the DY DT criteria is reported in table 5.6. 

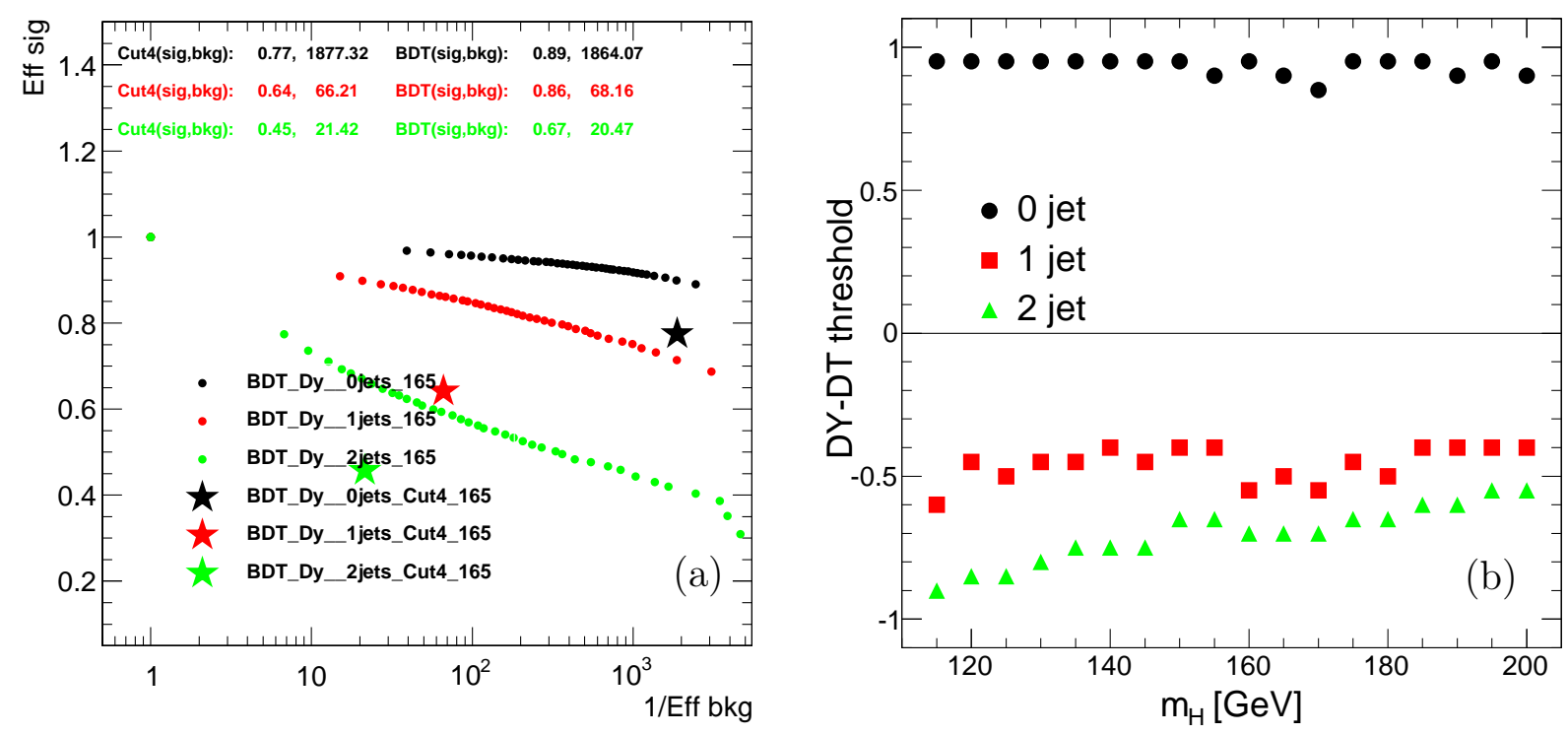

Figure 5.18: These two plots illustrate the thresholds used to cut on the output of the DY DT to reject the Drell-Yan background. The signal efficiency is shown as a function of the background inverse efficiency in (a), for a DY DT trained at $\mathrm{m}_{\mathrm{H}}=165 \mathrm{GeV}$. For each jet multiplicity, solid circles correspond to the efficiencies for several values of the threshold in the range $[-1,+1]$; solid stars indicate the efficiencies that can be obtained with the cut-based selection. For the same background inverse efficiency, the DY DT always provides higher signal efficiency than the cut-based selection. At each mass point, we choose the value of the DY DT threshold that matches the cut-based background inverse efficiency. These values are shown as a function of $\mathrm{m}_{\mathrm{H}}$ in figure (b). 


\begin{tabular}{|c|c|c|c|c|c|c|c|}
\hline \multirow[t]{2}{*}{ Sample } & \multirow{2}{*}{$\begin{array}{c}\text { Inclusive } \\
\text { presel }\end{array}$} & \multicolumn{2}{|c|}{0 Jets } & \multicolumn{2}{|c|}{1 Jets } & \multicolumn{2}{|c|}{2 Jets } \\
\hline & & presel & final & presel & final & presel & final \\
\hline Signal, $\mathrm{m}_{\mathrm{H}}=165 \mathrm{GeV}$ & 24.0 & 11.3 & 9.3 & 6.9 & 5.5 & 5.8 & 3.7 \\
\hline HWW & 13.7 & 9.4 & 8.2 & 3.6 & 3.2 & 0.77 & 0.69 \\
\hline $\mathrm{HZZ}$ & 1.2 & 0.39 & 0.07 & 0.43 & 0.06 & 0.42 & 0.02 \\
\hline WH & 3.5 & 0.73 & 0.47 & 1.2 & 0.96 & 1.6 & 1.2 \\
\hline $\mathrm{ZH}$ & 4.0 & 0.52 & 0.32 & 1.0 & 0.66 & 2.5 & 1.2 \\
\hline VBF & 1.5 & 0.26 & 0.22 & 0.71 & 0.64 & 0.58 & 0.52 \\
\hline $\mathrm{Z} / \gamma^{*} \rightarrow \mu^{+} \mu^{-}$ & 684570.0 & 590715.5 & 201.8 & 80333.2 & 969.1 & 13521.4 & 579.6 \\
\hline $\mathrm{Z} / \gamma^{*} \rightarrow \tau^{+} \tau^{-}$ & 5809.6 & 4995.3 & 2.7 & 696.4 & 109.8 & 117.8 & 46.8 \\
\hline $\mathrm{t} \overline{\mathrm{t}}$ & 363.5 & 9.5 & 3.8 & 95.6 & 76.4 & 258.3 & 209.4 \\
\hline $\mathrm{W}(+$ jets $/ \gamma)$ & 367.5 & 296.9 & 136.6 & 59.2 & 38.0 & 11.4 & 7.2 \\
\hline WW & 502.9 & 423.1 & 198.2 & 68.3 & 47.0 & 11.4 & 8.7 \\
\hline WZ & 308.3 & 94.5 & 22.8 & 109.4 & 16.3 & 104.4 & 9.6 \\
\hline $\mathrm{ZZ}$ & 269.5 & 80.0 & 19.6 & 83.1 & 11.1 & 106.4 & 9.9 \\
\hline Multijets & 1904.6 & 1470.2 & 104.2 & 346.6 & 45.6 & 87.9 & 19.5 \\
\hline Total background & 694095.8 & 598085.0 & 689.7 & 81791.7 & 1313.2 & 14219.1 & 890.8 \\
\hline Data & 685872.0 & 592539.0 & 612.0 & 79759.0 & 1420.0 & 13574.0 & 888.0 \\
\hline
\end{tabular}

Table 5.6: Expected and observed event yields for the $8.1 \mathrm{fb}^{-1}$ dataset, after preselection and at the final selection.

\subsection{Final Discriminant}

The variable used as a final discriminant for the $5.4 \mathrm{fb}^{-1}$ analysis is the output of a neural network. We use the TMultilayerPerceptron implementation [81] of the neural network discriminant with sigmoid activation function. The neural network is trained with a steepestdescent algorithm based on the Broyden-Fletcher-Goldfarb-Shanno method. The NN has thirteen input variables, listed in table 5.7, and one hidden layer with eight neurons. In the $5.4 \mathrm{fb}^{-1}$ analysis, the dataset is not split into the subsets corresponding to events with zero, one, and two or more jets. We therefore train one single neural network for each mass value that we consider. An example of the output distribution for the neural network discriminant is shown in figure 5.19.

The variable used as a final discriminant for the $8.1 \mathrm{fb}^{-1}$ analysis is the output of a decision tree. As mentioned in section 5.5.2, the configuration of the FD DT is almost the 


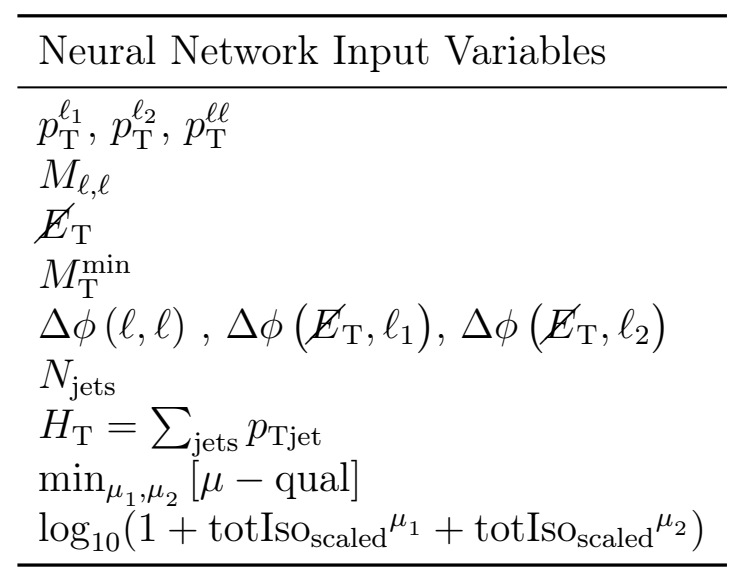

Table 5.7: Input variables for the neural network discriminant.
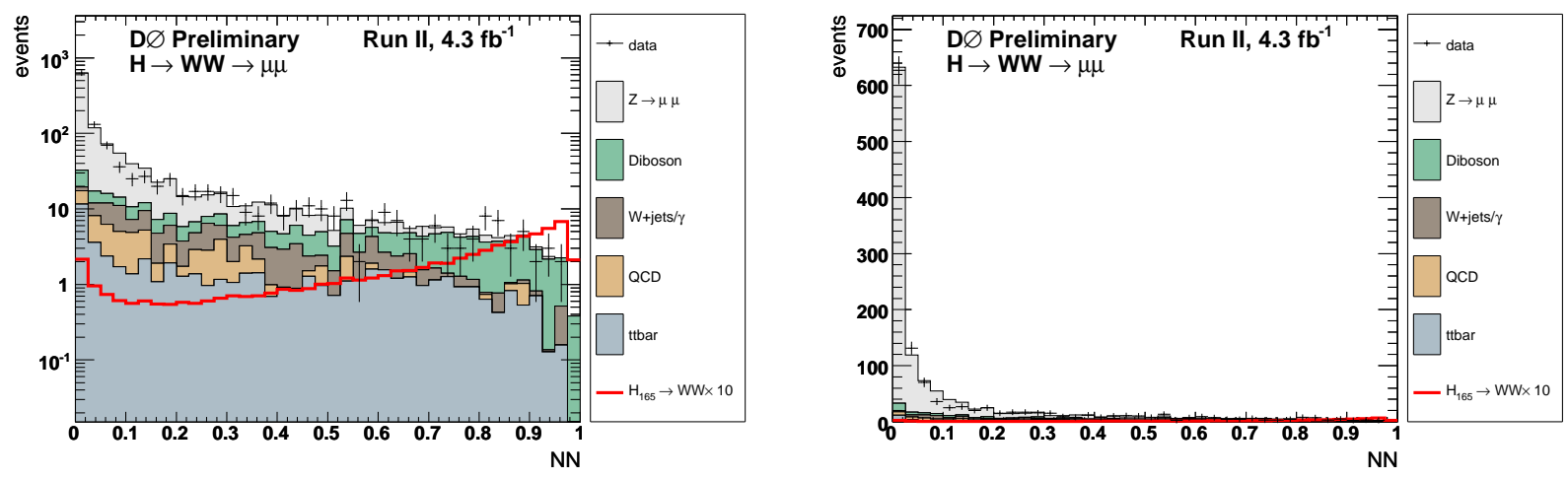

Figure 5.19: The neural network output distribution in logarithmic scale (left) and linear scale (right) at the final selection stage for the RunIIb dataset with $\mathrm{m}_{\mathrm{H}}=165 \mathrm{GeV}$. 
same one used for the DY DT. The final discriminant DT uses as its inputs all the variables used for the DY discriminant, with a few additional variables, as indicated in table 5.5. The Final Discriminant (FD) is trained separately in each jet bin and for each Higgs mass value using the events that are not suppressed by the DY DT rejection criteria. The small number of $\mathrm{Z} / \gamma^{*}$ events left after the DY DT rejection ensures that the FD DT training focuses on discriminating against the remaining signal-like backgrounds. These backgrounds, such as t $\overline{\mathrm{t}}$ and the non-resonant diboson production, can be identified by the multivariate discriminant with the additional input variables of the FD DT. The output of the FD is then used to search for the presence of a Higgs boson signal or to place limits on its production cross section. The distributions for RunIIb of the FD DT trained for a hypotetical Higgs boson with mass $\mathrm{m}_{\mathrm{H}}=165 \mathrm{GeV}$ are shown in figures 5.20 to 5.22
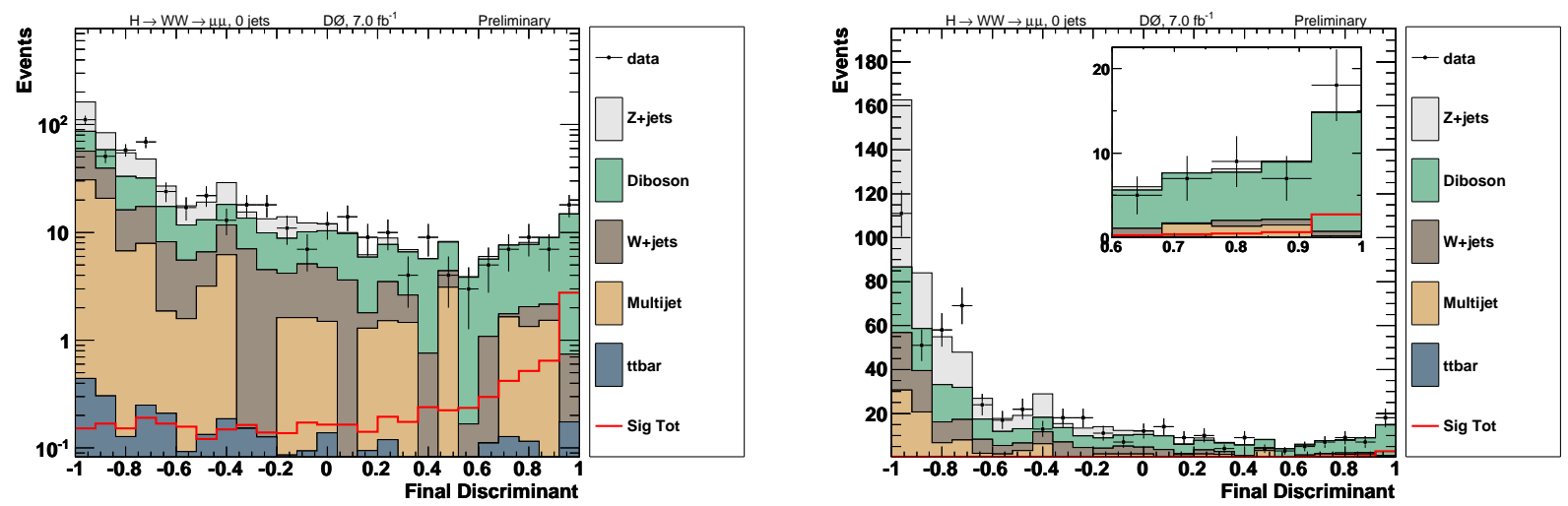

Figure 5.20: The FD DT distribution in logarithmic scale (left) and linear scale (right) at the final selection stage for the RunIIb 0-jet bin with $\mathrm{m}_{\mathrm{H}}=165 \mathrm{GeV}$. 

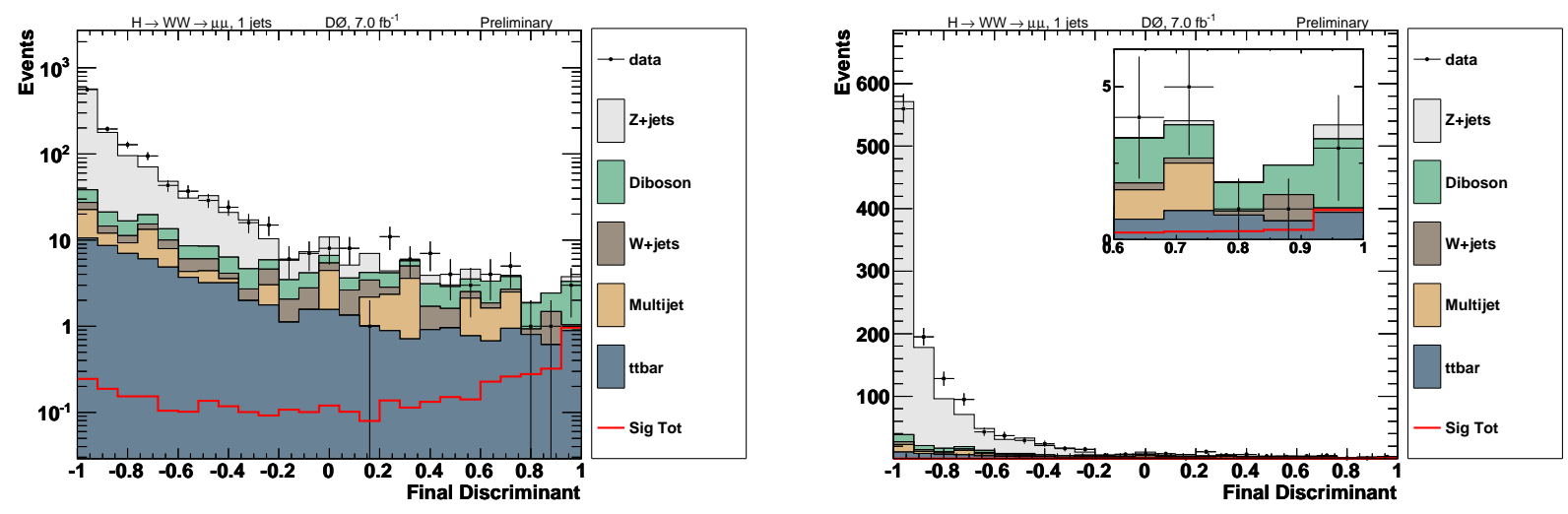

Figure 5.21: The FD DT distribution in logarithmic scale (left) and linear scale (right) at the final selection stage for the RunIIb 1-jet bin with $\mathrm{m}_{\mathrm{H}}=165 \mathrm{GeV}$.
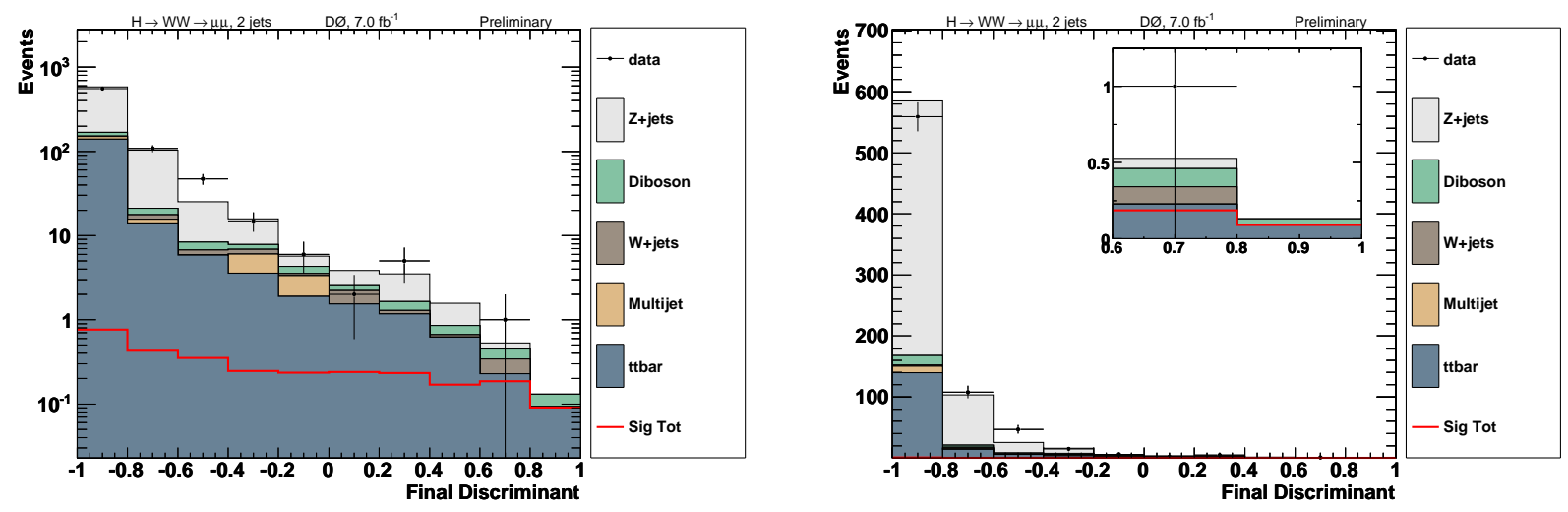

Figure 5.22: The FD DT distribution in logarithmic scale (left) and linear scale (right) at the final selection stage for the RunIIb 2-jet bin with $\mathrm{m}_{\mathrm{H}}=165 \mathrm{GeV}$. 


\section{CHAPTER 6 LIMIT CALCULATION}

For all of the Higgs boson mass values that we consider, we do not observe any excess of signal-like events in the data. We therefore proceed to compute upper limits on the SM cross section for Higgs boson production. The statistical significance of the result is computed using the $\mathrm{CL}_{s}$ method as implemented in the Confidence Level Limit Evaluator (COLLIE) software $^{1}$ package [82]. Using the formalism described in section 6.1 , the calculation of the limit takes into account both statistical uncertainties and systematic uncertainties; systematic uncertainties are described in section 6.2. The limits obtained in the $5.4 \mathrm{fb}^{-1} \mathrm{NN}$ analysis are illustrated in section 6.3; the limits obtained in the $8.1 \mathrm{fb}^{-1}$ DT analysis are illustrated in section 6.4. The results are illustrated for both the dimuon channel and for the combination of the three dilepton channels.

\subsection{Statistical Treatment}

The distribution of the final variable observed in data is compared to the distributions expected in two hypotheses: the null hypothesis and the test hypothesis. The backgroundonly distribution (B) is predicted in the null hypothesis that the SM Higgs boson does not

\footnotetext{
${ }^{1}$ We use version V00-03-17 for the NN result, and version V00-04-09 for the DT result.
} 
exist. The signal-plus-background distribution $(\mathrm{S}+\mathrm{B})$ is predicted in the test hypothesis that the Higgs boson does exist. An excess of signal-like events, with high values of the final discriminant, is expected in the $\mathrm{S}+\mathrm{B}$ hypothesis, as illustrated in figure 6.1. A test statistic
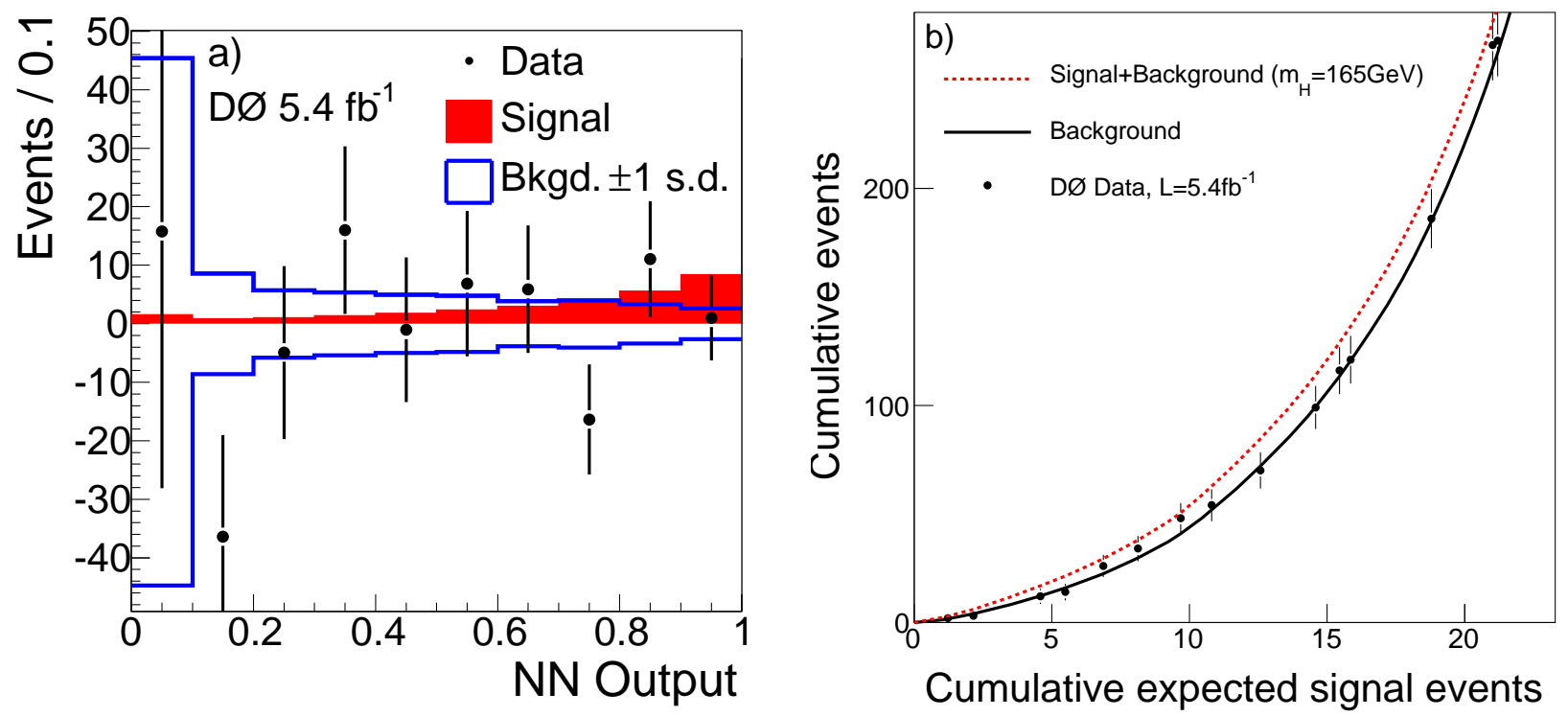

Figure 6.1: (a) Data after subtracting the fitted background (points) and SM signal expectation (filled histogram) as a function of the $\mathrm{NN}$ output for $\mathrm{m}_{\mathrm{H}}=165 \mathrm{GeV}$. Also shown is the \pm 1 standard deviation band on the total background after fitting; see text for details about the fitting procedure. (b) Total number of events vs. number of expected signal events, showing that there is no excess of signal-like events. The cumulative number of events is computed after re-ordering the bins of the final distribution according to their $s / b$ value, and then integrating from high $s / b$ to low $s / b$. Displaying cumulative numbers rather than bin contents, statistical fluctuations cancel one another, allowing for an easier visualization of a potential excess.

is a quantity that is computed from the observed data and that is used to quantify the extent to which the data is consistent with the null hypothesis or with the test hypothesis. For example, given the $\mathrm{S}+\mathrm{B}$ and $\mathrm{B}$ distributions, one can compute the Poisson likelihood ratio $\mathrm{Q}$ for the observed data:

$$
Q=\frac{p(\text { data } \mid \mathrm{S}+\mathrm{B})}{p(\text { data } \mid \mathrm{B})} .
$$


Because of numerical considerations, we rarely consider $Q$; instead, we consider the negative log-likelihood ratio (LLR):

$$
\operatorname{LLR}=-2 \log (Q)=2 \sum_{i=1}^{N_{\text {bins }}}\left(s_{i}-d_{i} \log \left(1+\frac{s_{i}}{b_{i}}\right)\right)
$$

where $d_{i}, s_{i}, b_{i}$ are the number of events in each bin for data, signal, and background. A positive LLR value indicates that the data is more compatible with the background-only hypothesis, whereas a negative LLR value indicates that the data is more compatible with the signal-plus-background hypothesis. From equation (6.2), the median expected LLR value for the background-only hypothesis can be computed by replacing $d_{i}$ with $b_{i}$; the median expected LLR value for the signal-plus-background hypothesis can be computed by replacing $d_{i}$ with $s_{i}+b_{i}$.

The LLR test statistic approximates a Gaussian $\chi^{2}$ function and, from its probability distribution, we can compute the confidence level (CL) of our result. To determine the confidence level for the signal exclusion, we use the $\mathrm{CL}_{s}$ method, which is described in Ref. [83; 84] and is also known as the modified frequentist approach. In general, the confidence level for signal exclusion is computed as the probability for the test hypothesis $\mathrm{S}+\mathrm{B}$ to produce an outcome that is more background-like than what is observed in data; this probability is indicated in figure 6.2 by $\mathrm{CL}_{s+b}$. With the $\mathrm{CL}_{s}$ method, conversely, the confidence level for signal exclusion is estimated from the ratio

$$
\mathrm{CL}_{s}=\frac{\mathrm{CL}_{s+b}}{\mathrm{CL}_{b}},
$$

whose numerator and denominator are illustrated in figure 6.2. Unlike $\mathrm{CL}_{s+b}$, the $\mathrm{CL}_{s}$ method prevents false exclusions that can occur when the data fluctuates significantly below the background-only prediction, in particular when the background has low statistics or is poorly modeled. We report our results at the $1-\mathrm{CL}_{s}=95 \%$ confidence level. They are presented either with the LLR plot or with the limit plot. The LLR plot, visible in figure 6.4(a) for 


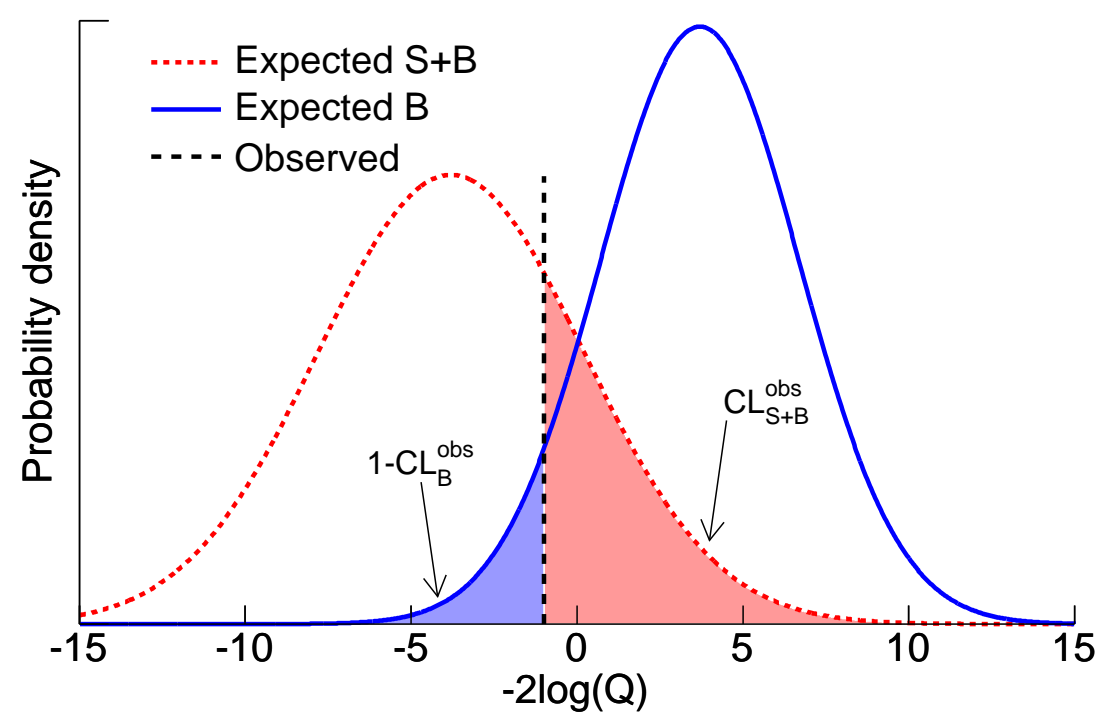

Figure 6.2: Illustration of the $\mathrm{CL}_{s}$ method. The expected sensitivity of the search corresponds to the separation between the median for the $\mathrm{S}+\mathrm{B}$ probability density distribution and the median for the $\mathrm{B}$ hypothesis. The confidence level for the absence of signal is computed, using the observed LLR value, as the ratio $\mathrm{CL}_{s+b} / \mathrm{CL}_{b}$.

example, shows the median LLR values for the $\mathrm{B}$ and $\mathrm{S}+\mathrm{B}$ hypotheses, as a function of the unknown parameter $\mathrm{m}_{\mathrm{H}}$. In other words, a slice of the LLR plot for a given value of $\mathrm{m}_{\mathrm{H}}$ corresponds to the figure 6.2: the separation between the expected $\mathrm{B}$ and $\mathrm{S}+\mathrm{B}$ curves indicates the sensitivity of the search, and the observed LLR curve indicates whether the data better agrees with the $\mathrm{B}$ hypothesis or with the $\mathrm{S}+\mathrm{B}$ one. In addition, the width of the probability density function for the background hypothesis is indicated by the bands corresponding to one and two standard deviations. The limit plot, shown for example in figure 6.4(b), is obtained by computing the multiplicative factor by which the cross section for SM Higgs boson production should be increased in order to reach the 95\% CL exclusion.

The procedure described so far only accounts for the Poissonian statistical uncertainty of each bin. Systematic uncertainties, described in section 6.2, account for the limited knowledge that we might have of parameters that are not of immediate interest in this study - called the nuisance parameters. The incorporation of systematic uncertainties in the calculation of confidence levels is not trivial, and its implementation is described in detail in the COLLIE documentation [82]. In practice, a large number of pseudo-experiments is generated. In them, the nuisance parameters are randomised according to their prior ${ }^{2}$ probability density

\footnotetext{
${ }^{2}$ The introduction of prior probability density distributions for the nuisance parameters makes this method not purely frequentist. Prior probability density distributions are assumed to be approximately Gaussian.
} 
distributions. Furthermore, an alternative test statistic, the profile likelihood, is used instead of the negative log-likelihood ratio. The profile likelihood is parametrized by the nuisance parameters, and is determined by a fit procedure performed in each pseudo-experiment. One important feature of the fit procedure is that, using the information available in the low- $s / b$ bins of the input distribution, the profiling technique allows for the determination of the value of the nuisance parameters. Thus, the fit procedure can often constrain the nuisance parameters better than our prior knowledge about their values.

\subsection{Systematic Uncertainties}

Two types of systematic uncertainties are considered: flat ones and shaped ones. Flat uncertainties affect the yield for a given process and therefore the normalization of the corresponding final distribution histogram. An example of a flat uncertainty is the uncertainty on the Higgs boson signal cross section. Shaped uncertainties affect the shape, or the shape and the normalization, of the final discriminant distribution for a given process. An example

of a shaped uncertainty is the uncertainty on the $p_{\mathrm{T}}^{\mathrm{Z}}$ spectrum for $\mathrm{Z} / \gamma^{*}(+$ jets $/ \gamma)$ samples. Below is a list of the flat uncertainties that we consider:

- The theoretical cross sections uncertainty is estimated to be $\pm 10 \%$ for the Higgs boson signal, $\pm 7 \%$ for the diboson background processes, $\pm 10 \%$ for the tet background, $\pm 6 \%$ for the $\mathrm{Z} / \gamma^{*}(+$ jets $/ \gamma)$ and $\pm 6 \%$ for $\mathrm{W}(+$ jets $/ \gamma)$.

- The muon identification efficiency is $\pm 8 \%$, as estimated by the MuonID group.

- The uncertainty on the proton and antiproton PDFs, which affects mostly the gg $\rightarrow \mathrm{H}$ cross section, is assigned to be $\pm 2.5 \%$.

- The uncertainty on the number of estimated multijet background events is assigned to be $\pm 20 \%$. This conservative value is based on the comparison of different parametrizations for the multijet estimate. 
- The systematic uncertainty due to the inclusive normalization factor $S$ is estimated to be $6 \%$. When the dataset is split into jet-multiplicity sub-samples, the uncertainty on the $\alpha_{i-j e t}$ factors is estimated to be $2 \%$ for $0-j e t, 6 \%$ for $1-j e t$, and $15 \%$ for $2-j e t$.

- The uncertanity from $b$-tagging is estimated to be $5 \%$ for the real b-jets and $10 \%$ for the fake b-jets or the light flavor jets.

Below is the list of the shaped uncertainties that we consider:

- The uncertainties due to the jet resolution and to the jet energy scale correction are provided by the jet group as a $\pm 1 \sigma$ variation of the nominal values.

- The uncertainty on the jet identification efficiency is calculated by decreasing the jet efficiency by one $\sigma$.

- The uncertainty on the factor correcting the vertex confirmation efficiency is calculated by decreasing this factor by one $\sigma$.

- The uncertainty on the muon $p_{\mathrm{T}}$ smearing is estimated by varying by $\pm 1 \sigma$ the parameters of the smearing function.

- The uncertainty on the reweighting applied to the $p_{\mathrm{T}}$ spectrum of the $\mathrm{Z}$ boson for $\mathrm{Z} / \gamma^{*}(+$ jets $/ \gamma)$ samples is calculated by shifting the parameters of the reweighting function by their $\pm 1 \sigma$ errors.

- The uncertainty on the reweighting applied to the $p_{\mathrm{T}}$ spectrum of the $\mathrm{W}$ boson for $\mathrm{W}(+$ jets $/ \gamma)$ samples is estimated by not applying the reweighting.

- The uncertainty on the reweighting applied to the $p_{\mathrm{T}}$ spectrum of the $\mathrm{W}$ bosons for the WW background is estimated by not applying the reweighting.

- The uncertainty on the reweighting applied to the $\Delta \phi(\ell, \ell)$ for the WW background is estimated by not symmetrizing $30 \%$ of the uncertainty resulting by not applying the reweighting. 
- For the gg $\rightarrow \mathrm{H}$ signal samples, we account for the uncertainty on the renormalization and factorization scales that affect the spectrum of Higgs boson $p_{\mathrm{T}}$. This uncertainty, illustrated in figure 4.1 , is estimated by varying $\mu_{R}$ and $\mu_{F}$ by $\pm 50 \%$ around their nominal value.

The results of the SM Higgs boson search are reported in section 6.3 for the NN study, and in section 6.4 for the DT study.

\subsection{Neural Network Results}

Here we report the results obtained with the NN study: the limits obtained for the SM Higgs boson are in section 6.3.1; the limits for the SM Higgs boson with a fourth generation of quarks are in section 6.3.2.

\subsubsection{Standard Model Results}

The maximum separation between the expected $\mathrm{LLR}_{b}$ and $\mathrm{LLR}_{s+b}$ curves is observed for $\mathrm{m}_{\mathrm{H}}=165 \mathrm{GeV}$, which corresponds to the mass region with the optimal sensitivity. This can be seen in the LLR plot of the NN analysis shown in figure 6.3(a). The observed LLR values are always consistent with the background-only hypothesis within one standard deviation. For $\mathrm{m}_{\mathrm{H}}$ values below $155 \mathrm{GeV}$, the observed LLR values are negative, and the data is more consitent with the $\mathrm{S}+\mathrm{B}$ hypothesis. Nevertheless, this excess of signal-like events is consistent with the fluctuations that one would expect within one standard deviation.

In the mass region with the optimal sensitivity, the dimuon channel alone is sensitive to a cross section that is three times the one predicted by the SM. The limit plot is shown in figure 6.3(b), and the corresponding limit values are reported in table 6.1.

The $5.4 \mathrm{fb}^{-1}$ neural network result obtained from the combination of the three channels ee, $e \mu$, and $\mu \mu$ is shown in figure 6.4. For high $\mathrm{m}_{\mathrm{H}}$, the observed LLR values fluctuate around the $L_{L R}$ curve; for low $m_{H}$, the observed LLR values are close to the $L_{L R}+b$ curve, indicating a 


\begin{tabular}{|c|c|c|c|c|c|c|c|c|c|c|c|c|c|c|c|c|c|c|}
\hline $\mathrm{m}_{\mathrm{H}}[\mathrm{GeV}]$ & 115 & 120 & 125 & 130 & 135 & 140 & 145 & 150 & 155 & 160 & 165 & 170 & 175 & 180 & 185 & 190 & 195 & 200 \\
\hline \multicolumn{19}{|l|}{$\mu \mu$} \\
\hline exp. & 37. & 23. & 15 & 9.6 & 9.6 & 7.5 & 7.7 & 5.6 & 5.5 & 2.9 & 2.8 & 3.1 & 3.8 & 4.9 & 5.8 & 8.2 & 9.8 & 13. \\
\hline obs. & 28. & 18. & 12 & 9.3 & 7.3 & 6.3 & 5.3 & 4.7 & 3.9 & 3.1 & 2.9 & 3.4 & 4.1 & 4.8 & 6.3 & 7.8 & 9.4 & 12 . \\
\hline \multicolumn{19}{|l|}{$\ell \ell$} \\
\hline exp. & 15. & 9.7 & 7.2 & 5. & 4.2 & 3.5 & 3.1 & 2.6 & 2.0 & 1.4 & 1.4 & 1.6 & 2.1 & 2.6 & 3.3 & 4.2 & 5.1 & 6.2 \\
\hline obs. & 21. & 14. & 8.8 & 6.6 & 6.4 & 5.2 & 3.9 & 3.3 & 3.2 & 1.8 & 1.5 & 2.0 & 1.9 & 2.1 & 3.2 & 3.2 & 5.8 & 5.5 \\
\hline
\end{tabular}

Table 6.1: Neural network $5.4 \mathrm{fb}^{-1}$ analysis: expected and observed limits for the dimuon channel, and for the combination of the three dilepton channels.
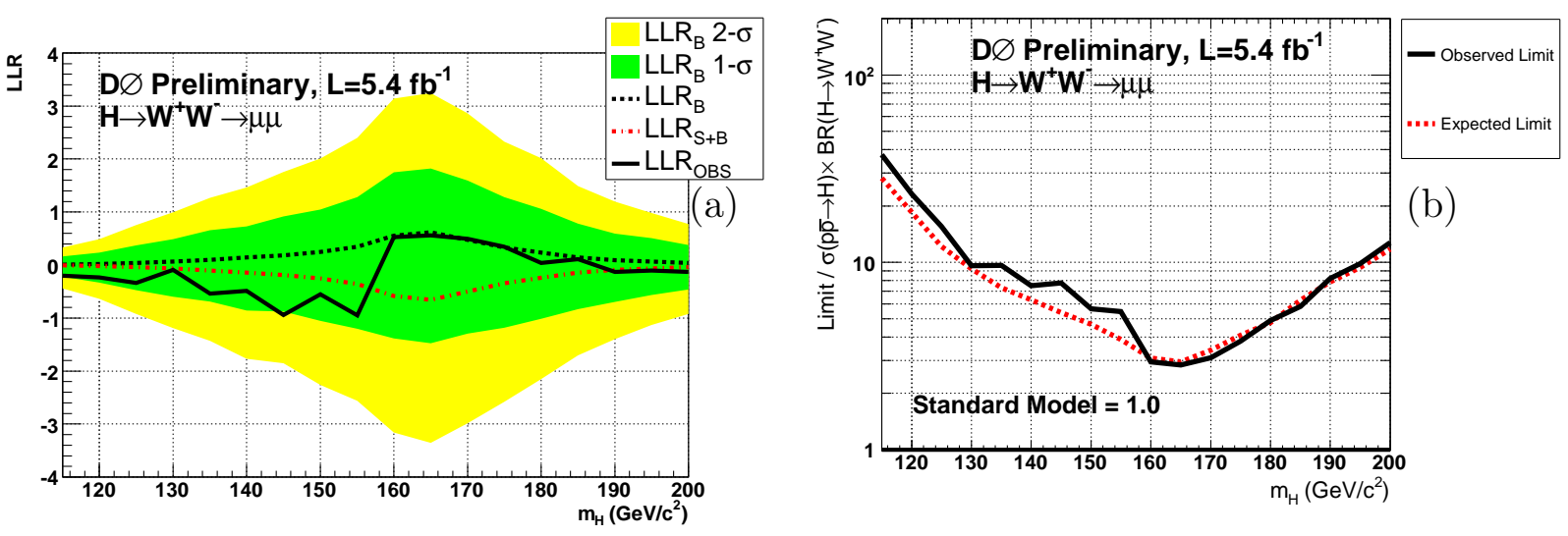

Figure 6.3: Dimuon channel: neural network analysis of the $5.4 \mathrm{fb}^{-1}$ dataset. (a) LLR plot: observed LLR, solid line, expected LLR for the background-only hypothesis, dashed black line, and signal-plus-background hypothesis, dotted red line. limit (a); Limit plot: expected limit, red dotted line, and observed limit, black solid line. (b).

potential upward fluctuation of the number of signal-like events. The combined D $\varnothing$ result is sensitive to a signal with a cross section that is 1.4 times bigger than that predicted by the $\mathrm{SM}$. The combination of the $\mathrm{D} \varnothing 5.4 \mathrm{fb}^{-1} \mathrm{NN}$ limit with the corresponding result from CDF is shown in figure 6.5; this combination is described in detail in Ref. [9]. The CDF result is based on a dataset of $4.8 \mathrm{fb}^{-1}$, and is described in Ref. [85]. This combination of the CDF and $\mathrm{D} \varnothing \mathrm{H} \rightarrow W^{+} W^{-}$analyses provids the first exclusion of a SM Higgs boson with mass in the range $162-166 \mathrm{GeV}$. 

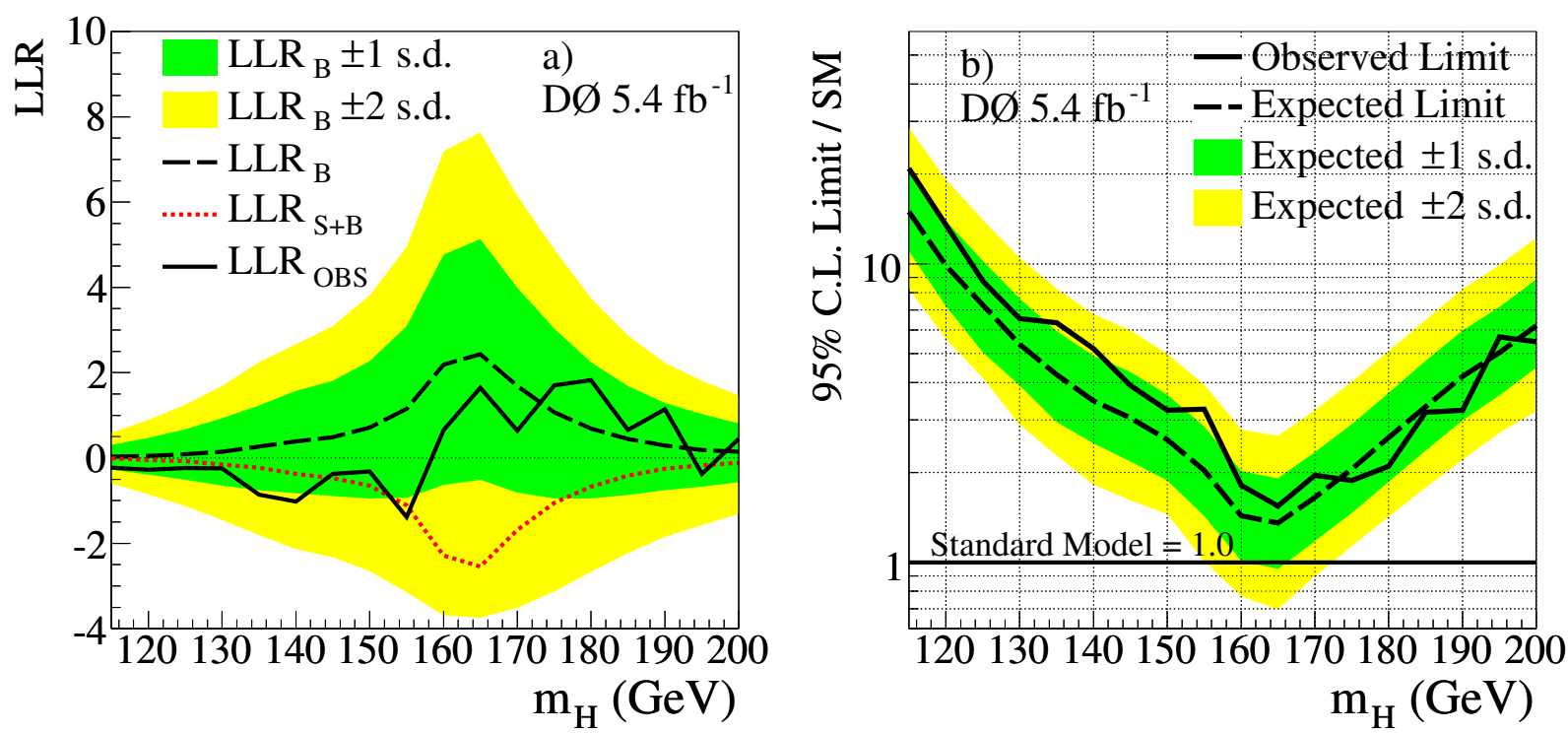

Figure 6.4: Combination of the $e e, e \mu$, and $\mu \mu$ channels: neural network analysis of the $5.4 \mathrm{fb}^{-1}$ dataset. (a) Observed LLR, solid line, expected LLR for background-only hypothesis, dashed line, and signal-plus-background hypothesis dotted line. (b) Upper limit on Higgs boson production cross section at 95\% C.L. expressed as a ratio to the SM cross section.

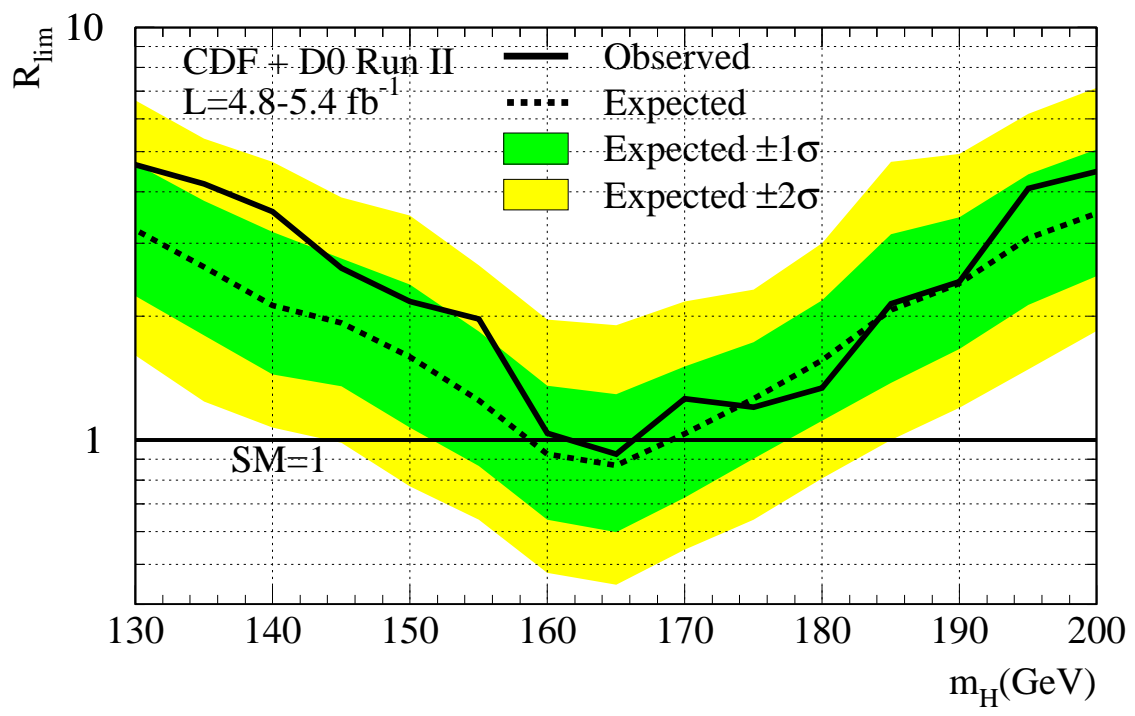

Figure 6.5: Combination of the $H \rightarrow W^{+} W^{-}$searches by CDF and D $\varnothing$. Upper limit on Higgs boson production cross section at 95\% C.L. expressed as a ratio to the SM cross section. The existence of a SM Higgs boson is excluded in the mass range $162-166 \mathrm{GeV}$. 


\subsubsection{Fourth-Generation Interpretation}

As described in section 1.3.2, the existence of a fourth generation of quarks would cause additional contributions to the gluon-gluon fusion process, enhancing significantly the gg $\rightarrow \mathrm{H}$ cross section. We interpret the $5.4 \mathrm{fb}^{-1} \mathrm{NN}$ result in this context, assuming the enhancement factors reported by Ref. [19; 86], and shown in figure 1.6. Within this interpretation, we consider only the gg $\rightarrow \mathrm{H}$ signal production, neglecting $\mathrm{VH}$ and $q q \rightarrow q q \mathrm{H}$; we also re-optimize the NN discriminant for the $\mathrm{gg} \rightarrow \mathrm{H}$ signal only. The resulting limit on $\sigma(\mathrm{gg} \rightarrow \mathrm{H})$ is shown in figure 6.6. We report the fourth-generation results as absolute limits on the value of

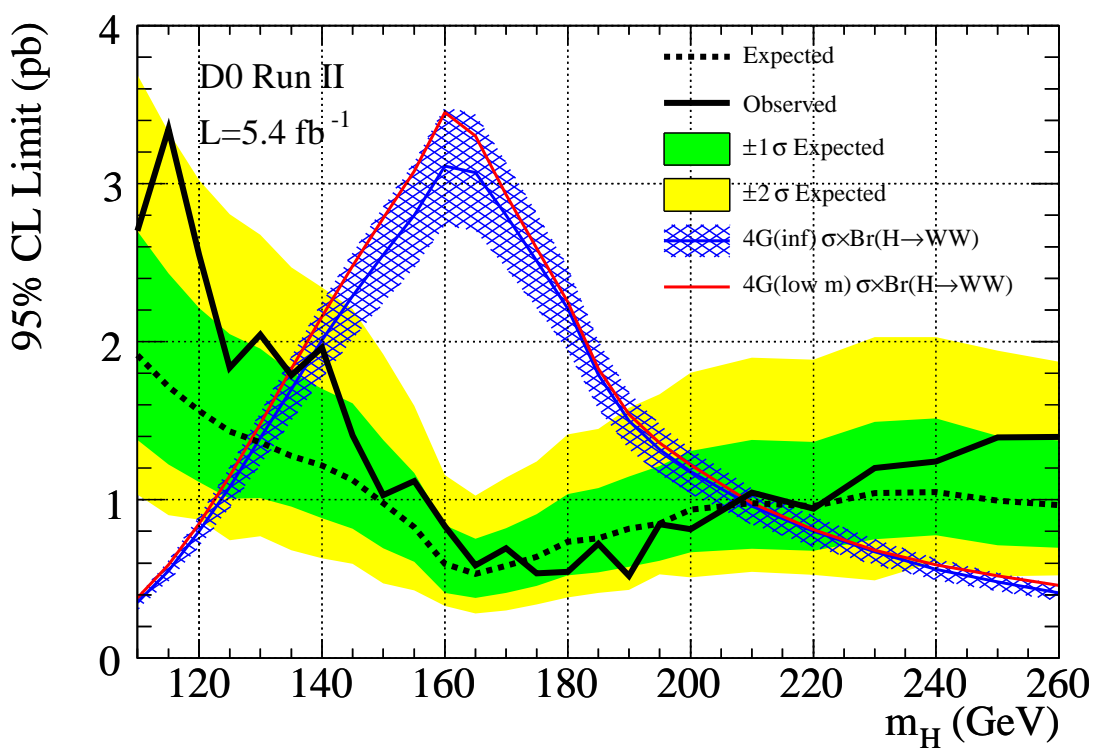

Figure 6.6: Combination of the $e e, e \mu$, and $\mu \mu$ channels: neural network analysis of the $5.4 \mathrm{fb}^{-1}$ dataset, fourth-generation interpretation [87]. 95\% C.L. limit on the absolute cross section for $\sigma\left(g g \rightarrow H \rightarrow \mathrm{W}^{+} \mathrm{W}^{-}\right)$. The shaded bands indicate the \pm 1 and \pm 2 standard deviation intervals on the distribution of the limits that are expected if a Higgs boson signal is not present. The error band on the fourth generation (4G) curve indicates the theoretical uncertainty on the cross section predicted. Also shown is the prediction for a fourth-generation model under the assumtion that the extra fermions are very heavy.

$\sigma(\mathrm{gg} \rightarrow \mathrm{H})$. Absolute limits are different from the relative limits that we report for the search with three quark generations. In the three-generations study, we express the limit as a relative scale factor on the total SM production because several signal contributions are involved, and the uncertainties on each signal contribution might differ. In the fourth-generation 
interpretation, we consider only one signal contribution, and can therefore set absolute limits on the value of $\sigma(\mathrm{gg} \rightarrow \mathrm{H})$

Following the assumption that a fourth generation of quarks exists, the $\mathrm{D} \varnothing H \rightarrow W^{+} W^{-}$ analysis alone can already exclude, at 95\% C.L., the existence of a SM Higgs boson with mass in the range $130-210 \mathrm{GeV}$. 


\subsection{Decision Trees Results}

In this section, we report the results obtained with the DT study. The $8.1 \mathrm{fb}^{-1}$ decision tree dimuon result, obtained from the combination of the three dimuon channels without jets, with one jet, and with two or more jets, is shown in figure 6.7. The corresponding numerical values are reported in table 6.2. The separate contributions of the three jet-multiplicity
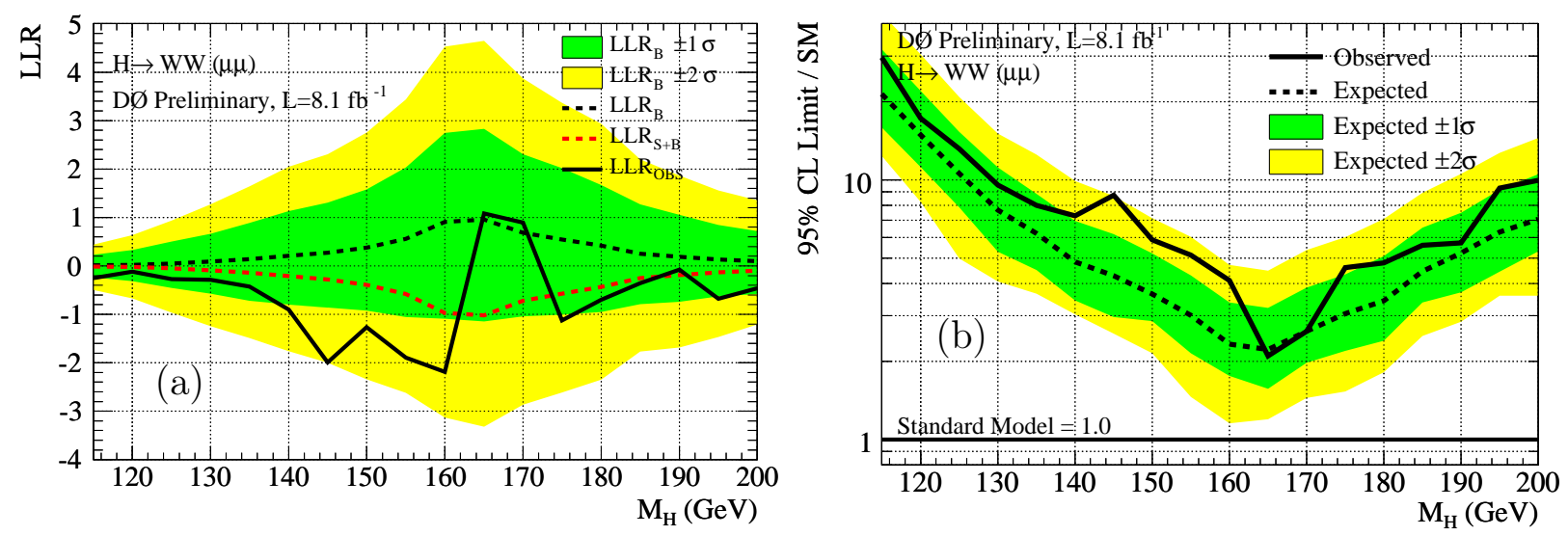

Figure 6.7: Dimuon channel: decision tree analysis of the $8.1 \mathrm{fb}^{-1}$ dataset. (a) LLR plot: observed LLR, solid line, expected LLR for the background-only hypothesis, dashed black line, and signal-plus-background hypothesis, dotted red line. (b) Limit plot: expected limit, dotted line, and observed limit, solid line.

\begin{tabular}{|c|c|c|c|c|c|c|c|c|c|c|c|c|c|c|c|c|c|c|}
\hline $\mathrm{m}_{\mathrm{H}}[\mathrm{GeV}]$ & 115 & 120 & 125 & 130 & 135 & 140 & 145 & 150 & 155 & 160 & 165 & 170 & 175 & 180 & 185 & 190 & 195 & 200 \\
\hline \multicolumn{19}{|l|}{$\mu \mu$} \\
\hline exp. & 21. & 15. & 11. & 7.6 & 6.2 & 4.8 & 4.3 & 3.6 & 3.0 & 2.3 & 2.2 & 2.6 & 3.0 & 3.4 & 4.4 & 5.2 & 6.3 & 7.1 \\
\hline obs. & 30. & 17. & 13. & 9.6 & 8.0 & 7.3 & 8.7 & 5.9 & 5.1 & 4.1 & 2.1 & 2.6 & 4.6 & 4.8 & 5.6 & 5.7 & 9.3 & 10. \\
\hline \multicolumn{19}{|l|}{$\ell \ell$} \\
\hline exp. & 8.5 & 5.8 & 4.4 & 3.4 & 2.7 & 2.2 & 1.9 & 1.7 & 1.4 & 1.1 & 0.97 & 1.1 & 1.3 & 1.6 & 1.9 & 2.2 & 2.7 & 3.2 \\
\hline obs. & 9.9 & 9.1 & 8.1 & 5.0 & 4.2 & 3.4 & 3.8 & 2.8 & 2.7 & 1.6 & 0.91 & 1.5 & 1.6 & 1.9 & 2.6 & 2.9 & 3.5 & 4.1 \\
\hline
\end{tabular}

Table 6.2: Decision tree $8.1 \mathrm{fb}^{-1}$ analysis: expected and observed limits for the dimuon channel, and for the combination of the three dilepton channels.

subsets to the dimuon result are shown in figure 6.8(a). The 0 - jet subset is the most sensitive across all the mass range, but the 1 - jet and $2^{+}$- jet channels also provide non-negligible contributions, in particular at very low and very high masses. The DT dimuon search is combined with the $e e$ and $e \mu$ searches; the contribution of each channel is shown in 

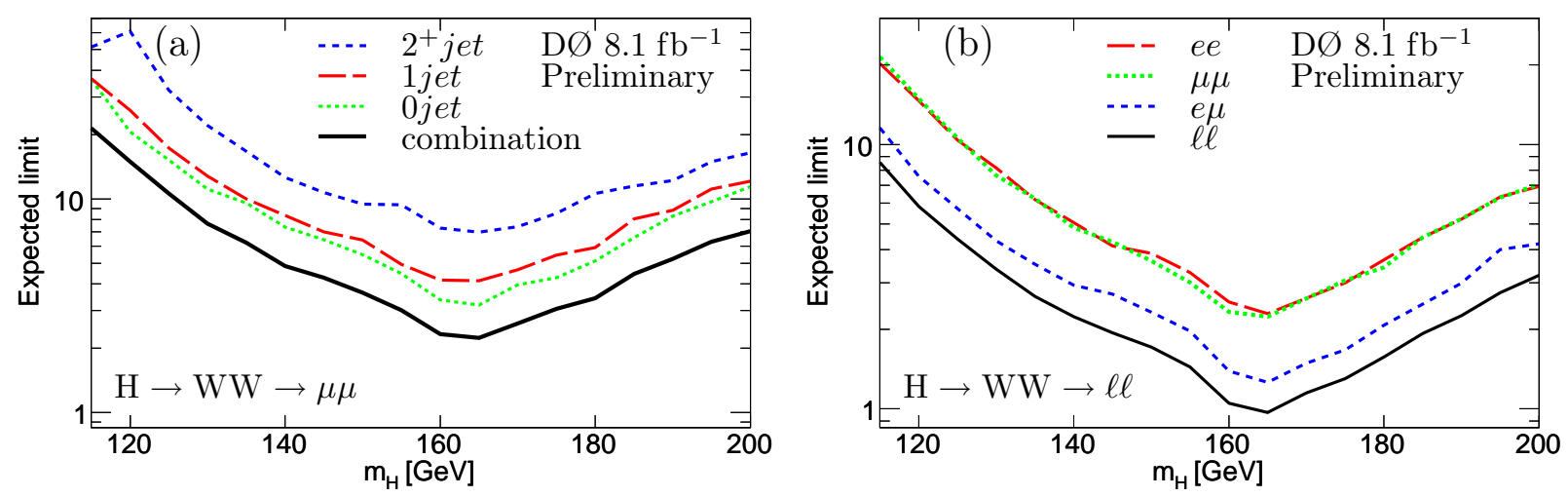

Figure 6.8: Decision tree analysis of the $8.1 \mathrm{fb}^{-1}$ dataset: (a) comparison of the expected limits for the three dimuon subsets with no jets, with one jets, with two or more jets, and of their combination; (b) comparison of the expected limits for the three dilepton channels ee, $e \mu, \mu \mu$, and of their combination $\ell \ell$

figure $6.8(\mathrm{~b})$; while the sensitivity of the dielectron channel is similar to the sensitivity of the dimuon channel, the $\mathrm{e}^{ \pm} \mu^{\mp}$ channel has a better sensitivity thanks to the lower number of $\mathrm{Z} / \gamma^{*}(+$ jets $/ \gamma)$ background events, and to the higher number of signal events. The higher number of signal events is due to the possible combinations of $\mathrm{WW}$ decays giving $\mathrm{e}^{ \pm} \mu^{\mp}$, which is twice that of $\mathrm{e}^{ \pm} \mu^{\mp}$ and $\mu^{+} \mu^{-}$. The combined $\mathrm{D} \varnothing$ limit plot is shown in figure 6.9.

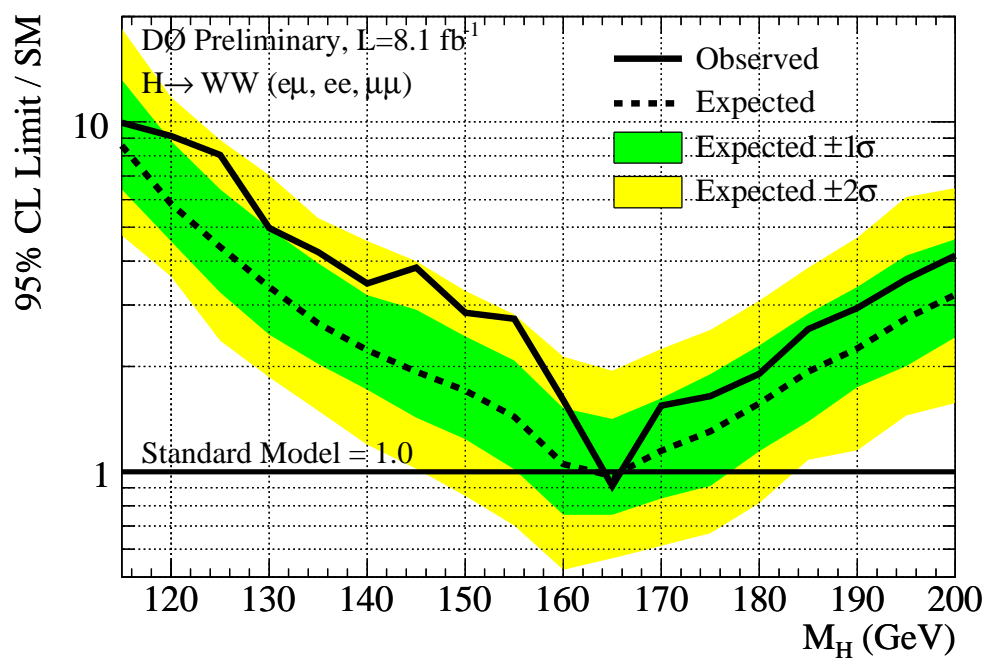

Figure 6.9: Decision tree analysis of the $8.1 \mathrm{fb}^{-1}$ dataset: combination of the three dilepton channels $e e, e \mu$, and $\mu \mu$. Observed (solid black line) and median expected (dashed black line) $95 \%$ C.L. upper limit on $\sigma\left(g g \rightarrow H \rightarrow \mathrm{W}^{+} \mathrm{W}^{-}\right)$.

As illustrated in figure 6.10, the DT search is more sensitive than the NN search, not 
only because of the additional $2.7 \mathrm{fb}^{-1}$ of data, but also thanks to the improved data analysis technique. The main improvements, described in chapter 5, are listed here as a reminder: splitting the analysis into three subsets with different jet multiplicities and background mixtures; more efficient DY background rejection by using a dedicated multivariate discriminant; improved signal isolation at low mass and high mass, where signal contributions different from gg $\rightarrow \mathrm{H}$ play an important role; relaxation of the primary vertex requirement through revertexing; relaxation of the $p_{\mathrm{T}}^{\mu_{1}}$ threshold; addition of input variables to the multivariate discriminant, such as $M_{\mathrm{T} 2}, M\left(j_{1} j_{2}\right)$, and $b$-tag information.

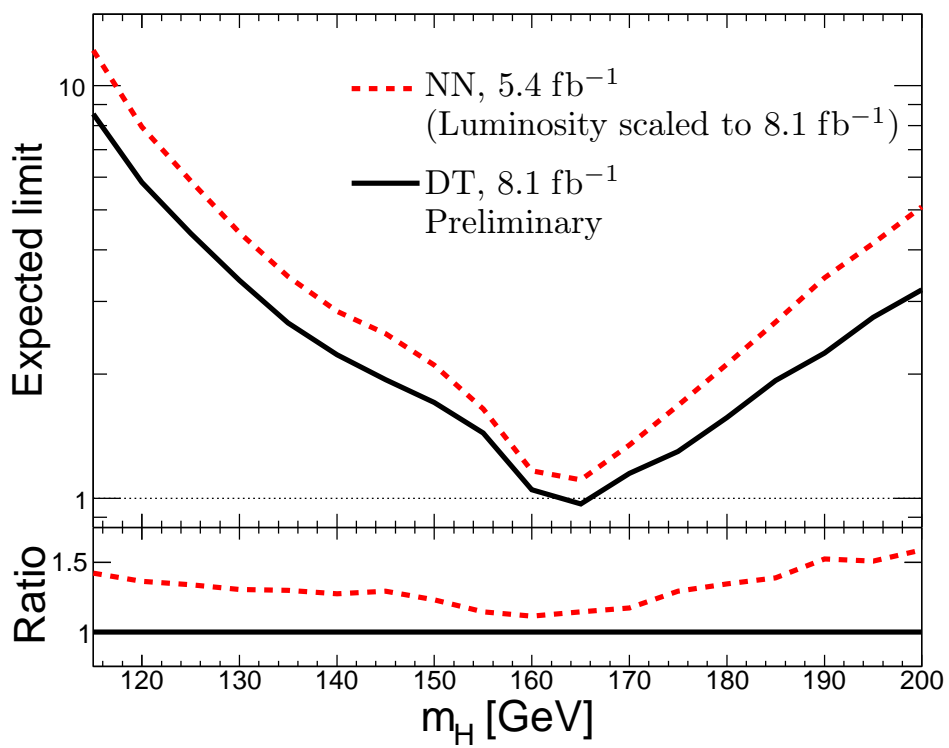

Figure 6.10: Comparison of the sensitivities obtained with the NN study and with the DT study. The NN result is scaled by the $\sqrt{L}$ factor that one would obtain by simply adding new data and decreasing the statistical uncertainty of the NN result published in Ref. [88]. The improvements for $\mathrm{m}_{\mathrm{H}}$ values in the vicinity of $165 \mathrm{GeV}$ are modest because, in this mass region, $\mathrm{gg} \rightarrow \mathrm{H}$ is the main signal contribution, for which the $\mathrm{NN}$ study was optimized. The improvements at low mass and high mass are more substantial because in these regions signal contributions other than $\mathrm{gg} \rightarrow \mathrm{H}$ become relevant.

With the $8.1-\mathrm{fb}^{-1} \mathrm{DT}$ analysis, the combination of the $e e, e \mu$, and $\mu \mu$ channels allows us to reach the SM sensitivity with the DØ dataset alone. The SM sensitivity would not be reached by simply increasing the dataset used in the NN study to $8.1 \mathrm{fb}^{-1}$, and without implementing the improvements performed in the DT study. The $8.1 \mathrm{fb}^{-1}$ DT search excludes 
the existence of a SM Higgs boson with $\mathrm{m}_{\mathrm{H}}=165 \mathrm{GeV}$, confirming the previous exclusion obtained from the combination of the CDF and $\mathrm{D} \varnothing$ analyses. 


\section{CHAPTER 7 CONCLUSION}

In this thesis, we report the results of the search for the SM Higgs boson performed at the DØ experiment with events that have two oppositely charged leptons and missing energy. This search was a remarkable success, although the Higgs boson was not found. For the first time, we were able to probe the high-mass range, and to exclude at 95\% CL the existence of the Higgs boson with a mass value of about $165 \mathrm{GeV}$. First, we reach the sensitivity required to test the hypothesis of a SM Higgs boson by combining the $H \rightarrow W^{+} W^{-}$searches at CDF and at $\mathrm{D} \varnothing$; we then reached the SM sensitivity with the $\mathrm{D} \varnothing$ search alone. The results of this search are also interpreted under the additional assumption that a fourth generation of quarks might exist, in which case we can exclude the SM Higgs boson hypothesis over a wider mass range.

Although other searches for the SM Higgs boson at the Tevatron may become available in the near future, they are less sensitive than the $\mathrm{H} \rightarrow \mathrm{W}^{+} \mathrm{W}^{-} \rightarrow \bar{\ell} \nu \ell \bar{\nu}$ one. Furthermore, the Tevatron collider is scheduled to end its operation in September 2011. In the context of the $\mathrm{D} \varnothing$ experiment, the results reported here thus constitute the definitive answer to the question of the existence of the high-mass SM Higgs boson. The latest combination of the $\mathrm{CDF}$ and $\mathrm{D} \varnothing$ high-mass searches is shown in figure 7.1. 


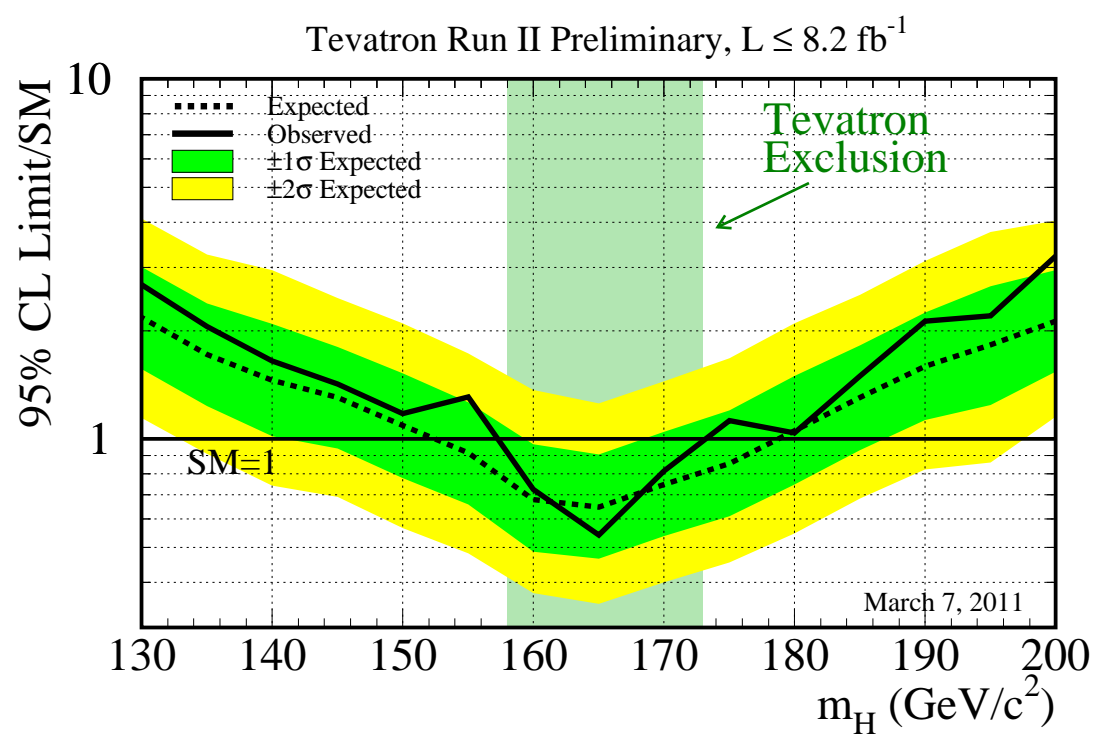

Figure 7.1: Latest Tevatron combination of the high-mass SM Higgs searches, as of March 2011. From Ref. [89].

The Large Hadron Collider in Geneva, Switzerland, recently re-started its operation, and the LHC experiments are now beginning to confirm the exclusion established by the CDF and $\mathrm{D} \varnothing$ searches. With additional data, the LHC experiments will be able to expand the mass range excluded by the Tevatron. 



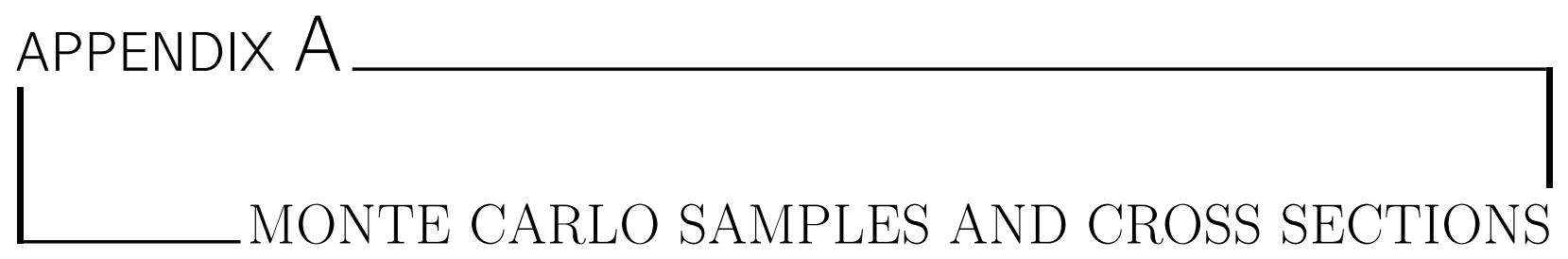

A.1 Signal Samples and Cross Sections 
Table A.1: Signal cross sections for the productions processes considered.

\begin{tabular}{lcccc}
\hline $\mathrm{m}_{\mathrm{H}}[\mathrm{GeV}]$ & $\mathrm{gg} \rightarrow \mathrm{H}[\mathrm{fb}]$ & WH $[\mathrm{fb}]$ & $\mathrm{ZH}[\mathrm{fb}]$ & $q q \rightarrow q q \mathrm{H}[\mathrm{fb}]$ \\
\hline 110 & 1385.0 & 212.00 & 125.7 & 85.1 \\
115 & 1215.9 & 174.50 & 103.9 & 78.6 \\
120 & 1072.3 & 150.10 & 90.2 & 72.7 \\
125 & 949.3 & 129.50 & 78.5 & 67.1 \\
130 & 842.9 & 112.00 & 68.5 & 62.1 \\
135 & 750.8 & 97.20 & 60.0 & 57.5 \\
140 & 670.6 & 84.60 & 52.7 & 53.2 \\
145 & 600.6 & 73.70 & 46.3 & 49.4 \\
150 & 539.1 & 64.40 & 40.8 & 45.8 \\
155 & 484.0 & 56.20 & 35.9 & 42.4 \\
160 & 432.3 & 48.50 & 31.4 & 39.4 \\
165 & 383.7 & 43.60 & 28.4 & 36.6 \\
170 & 344.0 & 38.50 & 25.3 & 34.0 \\
175 & 309.7 & 34.00 & 22.5 & 29.4 \\
180 & 279.2 & 30.10 & 20.0 & 27.3 \\
185 & 252.1 & 26.90 & 17.9 & 25.4 \\
190 & 228.0 & 24.00 & 16.1 & 23.7 \\
195 & 207.2 & 21.40 & 14.4 & 22.0 \\
200 & 189.1 & 19.10 & 13.0 & 19.1 \\
210 & 158.9 & 15.20 & 10.5 & 16.6 \\
220 & 134.5 & 12.30 & 8.5 & 14.5 \\
230 & 114.7 & 9.90 & 7.0 & 12.6 \\
240 & 98.4 & 8.03 & 5.7 & 11.0 \\
250 & 85.0 & 6.53 & 4.7 & 9.6 \\
260 & 73.8 & 5.33 & 3.9 & 8.4 \\
270 & 64.5 & 4.37 & 3.2 & 6.4 \\
280 & 56.7 & 3.59 & 2.7 & 5.6 \\
290 & 50.1 & 2.96 & 2.2 & \\
300 & 44.7 & 2.45 & 1.9 & \\
\hline & & & & \\
\hline
\end{tabular}


Table A.2: Higgs boson decay branching fractions computed with HDECAY.

\begin{tabular}{|c|c|c|}
\hline $\mathrm{m}_{\mathrm{H}}[\mathrm{GeV}]$ & $\mathrm{BR}\left(H \rightarrow W^{+} W^{-}\right)[\%]$ & $\mathrm{BR}(H \rightarrow \mathrm{ZZ})[\%]$ \\
\hline 110 & 4.585 & 0.4160 \\
\hline 115 & 8.268 & 0.8298 \\
\hline 120 & 13.64 & 1.527 \\
\hline 125 & 20.78 & 2.549 \\
\hline 130 & 29.43 & 3.858 \\
\hline 135 & 39.10 & 5.319 \\
\hline 140 & 49.16 & 6.715 \\
\hline 145 & 59.15 & 7.771 \\
\hline 150 & 68.91 & 8.143 \\
\hline 155 & 78.92 & 7.297 \\
\hline 160 & 90.48 & 4.185 \\
\hline 165 & 95.91 & 2.216 \\
\hline 170 & 96.39 & 2.351 \\
\hline 175 & 95.81 & 3.204 \\
\hline 180 & 93.25 & 5.937 \\
\hline 185 & 84.50 & 14.86 \\
\hline 190 & 78.70 & 20.77 \\
\hline 195 & 75.88 & 23.66 \\
\hline 200 & 74.26 & 25.33 \\
\hline 210 & 72.50 & 27.16 \\
\hline 220 & 71.60 & 28.11 \\
\hline 230 & 71.05 & 28.70 \\
\hline 240 & 70.66 & 29.11 \\
\hline 250 & 70.36 & 29.43 \\
\hline 260 & 70.12 & 29.70 \\
\hline 270 & 69.91 & 29.92 \\
\hline 280 & 69.72 & 30.12 \\
\hline 290 & 69.56 & 30.29 \\
\hline 300 & 69.40 & 30.45 \\
\hline
\end{tabular}




\section{A.2 Background Samples and Cross Sections}


Table A.3: $\mathrm{Z} \rightarrow \mu \mu+$ jets samples generated with ALPGEN along with corresponding initial number of events, matched ALPGEN LO cross sections times branching fractions, and NLO $k$-factor.

\begin{tabular}{|c|c|c|c|}
\hline Process & $(Z / \gamma$ Mass $)$ & $\sigma \times \mathrm{BF}[p b]$ & $\mathrm{K}$-factor \\
\hline $\mathrm{Z} \rightarrow \mu \mu+0 \mathrm{lp}$ & $(15-75)$ & 338.2 & \multirow{16}{*}{1.3} \\
\hline $\mathrm{Z} \rightarrow \mu \mu+1 \mathrm{lp}$ & $(15-75)$ & 40.02 & \\
\hline $\mathrm{Z} \rightarrow \mu \mu+2 \mathrm{lp}$ & $(15-75)$ & 10.04 & \\
\hline $\mathrm{Z} \rightarrow \mu \mu+\geq 3 \mathrm{lp}$ & $(15-75)$ & 2.755 & \\
\hline $\mathrm{Z} \rightarrow \mu \mu+\overline{0 l p}$ & $(75-130)$ & 133.4 & \\
\hline $\mathrm{Z} \rightarrow \mu \mu+1 \mathrm{lp}$ & $(75-130)$ & 40.29 & \\
\hline $\mathrm{Z} \rightarrow \mu \mu+2 \mathrm{lp}$ & $(75-130)$ & 9.993 & \\
\hline $\mathrm{Z} \rightarrow \mu \mu+\geq 3 \mathrm{lp}$ & $(75-130)$ & 3.094 & \\
\hline $\mathrm{Z} \rightarrow \mu \mu+0 \mathrm{lp}$ & $(130-250)$ & 0.8645 & \\
\hline $\mathrm{Z} \rightarrow \mu \mu+1 \mathrm{lp}$ & $(130-250)$ & 0.3728 & \\
\hline $\mathrm{Z} \rightarrow \mu \mu+2 \mathrm{lp}$ & $(130-250)$ & 0.09535 & \\
\hline $\mathrm{Z} \rightarrow \mu \mu+\geq 3 \mathrm{lp}$ & $(130-250)$ & 0.03187 & \\
\hline $\mathrm{Z} \rightarrow \mu \mu+\overline{0 l p}$ & $(250-1960)$ & 0.06919 & \\
\hline $\mathrm{Z} \rightarrow \mu \mu+1 \mathrm{lp}$ & $(250-1960)$ & 0.03438 & \\
\hline $\mathrm{Z} \rightarrow \mu \mu+2 \mathrm{lp}$ & $(250-1960)$ & 0.01198 & \\
\hline $\mathrm{Z} \rightarrow \mu \mu+\geq 3 \operatorname{lp}$ & $(250-1960)$ & 0.003899 & \\
\hline $\mathrm{Z} \rightarrow \mu \mu+2 b+0 \mathrm{lp}$ & $(15-75)$ & 0.5087 & \multirow{12}{*}{$1.3 \times 1.52$} \\
\hline $\mathrm{Z} \rightarrow \mu \mu+2 b+1 \mathrm{lp}$ & $(15-75)$ & 0.1988 & \\
\hline $\mathrm{Z} \rightarrow \mu \mu+2 b+\geq 2 \mathrm{lp}$ & $(15-75)$ & 0.07835 & \\
\hline $\mathrm{Z} \rightarrow \mu \mu+2 b+0 \mathrm{lp}$ & $(75-130)$ & 0.4242 & \\
\hline $\mathrm{Z} \rightarrow \mu \mu+2 b+1 \mathrm{lp}$ & $(75-130)$ & 0.1953 & \\
\hline $\mathrm{Z} \rightarrow \mu \mu+2 b+\geq 2 \mathrm{lp}$ & $(75-130)$ & 0.0990 & \\
\hline $\mathrm{Z} \rightarrow \mu \mu+2 b+0 \mathrm{lp}$ & $(130-250)$ & 0.003401 & \\
\hline $\mathrm{Z} \rightarrow \mu \mu+2 b+1 \mathrm{lp}$ & $(130-250)$ & 0.001844 & \\
\hline $\mathrm{Z} \rightarrow \mu \mu+2 b+\geq 2 \mathrm{lp}$ & $(130-250)$ & 0.0008843 & \\
\hline $\mathrm{Z} \rightarrow \mu \mu+2 b+0 \mathrm{lp}$ & $(250-1960)$ & 0.0003403 & \\
\hline $\mathrm{Z} \rightarrow \mu \mu+2 b+1 \mathrm{lp}$ & $(250-1960)$ & 0.0001730 & \\
\hline $\mathrm{Z} \rightarrow \mu \mu+2 b+\geq 2 \mathrm{lp}$ & $(250-1960)$ & 0.0001063 & \\
\hline $\mathrm{Z} \rightarrow \mu \mu+2 c+0 \mathrm{lp}$ & $(15-75)$ & 4.144 & \multirow{12}{*}{$1.3 \times 1.67$} \\
\hline $\mathrm{Z} \rightarrow \mu \mu+2 c+1 \mathrm{lp}$ & $(15-75)$ & 0.9530 & \\
\hline $\mathrm{Z} \rightarrow \mu \mu+2 c+\geq 2 \mathrm{lp}$ & $(15-75)$ & 0.3431 & \\
\hline $\mathrm{Z} \rightarrow \mu \mu+2 c+0 \mathrm{lp}$ & $(75-130)$ & 0.9322 & \\
\hline $\mathrm{Z} \rightarrow \mu \mu+2 c+1 \mathrm{lp}$ & $(75-130)$ & 0.5482 & \\
\hline $\mathrm{Z} \rightarrow \mu \mu+2 c+\geq 2 \mathrm{lp}$ & $(75-130)$ & 0.2808 & \\
\hline $\mathrm{Z} \rightarrow \mu \mu+2 c+0 \mathrm{lp}$ & $(130-250)$ & 0.007555 & \\
\hline $\mathrm{Z} \rightarrow \mu \mu+2 c+1 \mathrm{lp}$ & $(130-250)$ & 0.004394 & \\
\hline $\mathrm{Z} \rightarrow \mu \mu+2 c+\geq 2 \mathrm{lp}$ & $(130-250)$ & 0.002826 & \\
\hline $\mathrm{Z} \rightarrow \mu \mu+2 c+0 \mathrm{lp}$ & $(250-1960)$ & 0.0006222 & \\
\hline $\mathrm{Z} \rightarrow \mu \mu+2 c+1 \mathrm{lp}$ & $(250-1960)$ & 0.0004394 & \\
\hline $\mathrm{Z} \rightarrow \mu \mu+2 c+\geq 2 \mathrm{lp}$ & $(250-1960)$ & 0.0002619 & \\
\hline
\end{tabular}


Table A.4: $\mathrm{Z} \rightarrow \tau \tau+$ jets samples generated with ALPGEN + PyThIA along with corresponding initial number of events, matched ALPGEN LO cross sections times branching fractions, and NLO $k$-factor.

\begin{tabular}{lccc}
\hline Process & $(Z / \gamma$ Mass $)$ & $\sigma \times$ BF $[p b]$ & K-factor \\
\hline$Z \rightarrow \tau \tau+0 l p$ & $(15-75)$ & 336.5 & \\
$Z \rightarrow \tau \tau+1 l p$ & $(15-75)$ & 40.02 & \\
$Z \rightarrow \tau \tau+2 l p$ & $(15-75)$ & 9.938 & \\
$Z \rightarrow \tau \tau+\geq 3 l p$ & $(15-75)$ & 2.776 & \\
$Z \rightarrow \tau \tau+0 l p$ & $(75-130)$ & 133.1 & 1.3 \\
$Z \rightarrow \tau \tau+11 p$ & $(75-130)$ & 40.28 & \\
$Z \rightarrow \tau \tau+2 l p$ & $(75-130)$ & 10.00 & \\
$Z \rightarrow \tau \tau+\geq 3 l p$ & $(75-130)$ & 3.294 & \\
$Z \rightarrow \tau \tau+0 l p$ & $(130-250)$ & 0.8846 & \\
$Z \rightarrow \tau \tau+1 l p$ & $(130-250)$ & 0.3399 & \\
$Z \rightarrow \tau \tau+2 l p$ & $(130-250)$ & 0.0993 & \\
$Z \rightarrow \tau \tau+\geq 3 l p$ & $(130-250)$ & 0.0322 & \\
$Z \rightarrow \tau \tau+0 l p$ & $(250-1960)$ & 0.0658 & \\
$Z \rightarrow \tau \tau+11 p$ & $(250-1960)$ & 0.0353 & \\
$Z \rightarrow \tau \tau+2 l p$ & $(250-1960)$ & 0.0113 & \\
$Z \rightarrow \tau \tau+\geq 3 l p$ & $(250-1960)$ & 0.00388 &
\end{tabular}

Table A.5: $\mathrm{W} \rightarrow \ell \nu+$ jets samples generated with ALPGEN+PYTHIA along with corresponding initial number of events, matched ALPGEN LO cross sections times branching fractions, and $k$-factor.

\begin{tabular}{lcc}
\hline Process & $\sigma \times \mathrm{BF}[p b]$ & K-factor \\
\hline $\mathrm{W} \rightarrow \ell \nu+0 \mathrm{lp}$ & 4597 & \\
$\mathrm{~W} \rightarrow \ell \nu+1 \mathrm{lp}$ & 1234 & \\
$\mathrm{~W} \rightarrow \ell \nu+2 \mathrm{lp}$ & 301.8 & 1.3 \\
$\mathrm{~W} \rightarrow \ell \nu+3 \mathrm{lp}$ & 72.62 & \\
$\mathrm{~W} \rightarrow \ell \nu+4 \mathrm{lp}$ & 16.57 & \\
$\mathrm{~W} \rightarrow \ell \nu+\geq 5 \mathrm{lp}$ & 5.010 & \\
$\mathrm{~W} \rightarrow \ell \nu+2 b+0 \mathrm{lp}$ & 9.494 & \\
$\mathrm{~W} \rightarrow \ell \nu+2 b+1 \mathrm{lp}$ & 4.155 & \\
$\mathrm{~W} \rightarrow \ell \nu+2 b+2 \mathrm{lp}$ & 1.611 & \\
$\mathrm{~W} \rightarrow \ell \nu+2 b+\geq 3 \mathrm{lp}$ & 0.7478 & \\
$\mathrm{~W} \rightarrow \ell \nu+2 c+0 \mathrm{lp}$ & 23.37 & \\
$\mathrm{~W} \rightarrow \ell \nu+2 c+1 \mathrm{lp}$ & 13.48 & \\
$\mathrm{~W} \rightarrow \ell \nu+2 c+2 \mathrm{lp}$ & 5.527 & \\
$\mathrm{~W} \rightarrow \ell \nu+2 c+\geq 3 \mathrm{lp}$ & 2.408 & \\
\hline
\end{tabular}


Table A.6: Diboson and t $\bar{t}$ samples generated with PythiA along with the corresponding cross sections.

\begin{tabular}{lc}
\hline Process & $\sigma[\mathrm{pb}]$ \\
\hline$W W \rightarrow$ inclusive & 11.66 \\
$W Z \rightarrow$ inclusive & 3.45 \\
$Z Z \rightarrow$ inclusive & 1.37 \\
$\mathrm{t} \overline{\mathrm{t}} \rightarrow$ inclusive & 7.88 \\
\hline
\end{tabular}




\section{APPENDIX B}

\section{MONTE CARLO REWEIGHTINGS}

An overview of the reweightings being applied to the MC samples is shown in appendix B. The reweightings that are centrally provided to the D $\varnothing$ collaborators by the VJets group are indicated with "VJets". The VJets reweightings are documented in the references provided in the text. The other reweightings are specific to this search. The Z- $p_{\mathrm{T}}$ and $\mathrm{W}-p_{\mathrm{T}}$ reweightings are described in section 5.3.2.

The instantaneous luminosity $\mathcal{L}$ and the beamspot $z$-position reweightings are applied to correct potential differeces between the distributions observed in data and MC for these two quantities. The distributions for $\mathcal{L}$ and for the the beamspot $z$-position are determined in $\mathrm{MC}$ by the data events used for the zero-bias overlay. Differences in the instantaneous luminosity profile can cause data-MC discrepancies in the calorimeter noise and in the tracking efficiency. Differences in the beamspot $z$-position can cause data-MC discrepancies in the tracking acceptance.

For the diboson WW sample, a $\Delta \phi(\ell, \ell)$ reweighting is applied in addition to the WW $p_{\mathrm{T}}$ reweighting discussed in section 5.3.2. The $\Delta \phi(\ell, \ell)$ reweightings accounts for the fact that the PYTHIA-generated samples include the $q q \rightarrow \mathrm{WW}$ contribution, but not the gg $\rightarrow$ WW contribution. 
All the reweightings computed from data are derived in the same way. The histograms containing the distribution of the variables for which the reweighting is being computed are rebinned. The bins have variable width, and their width is chosen so that the number of events in each bin is sufficient to avoid large statistical fluctuations. The bin-by-bin ratio of data/MC is then computed, and the resulting histogram is modified by a smoothing algorithm.

The reweighting of the jet $\eta$ and $\Delta \mathrm{R}\left(j_{1}, j_{2}\right)$ distributions is attributed to a limitation of the AlPGEn generator. The jet reweightings are applied to the $\mathrm{Z} / \gamma^{*}$ samples only.

\begin{tabular}{lcc}
\hline Reweighting & Samples & Notes \\
\hline Z- $p_{\mathrm{T}}$ inclusive & $\mathrm{Z} / \gamma^{*}(+$ jets $/ \gamma)$ & VJets \\
Z- $p_{\mathrm{T}}$ N-jet dep. & $\mathrm{Z} / \gamma^{*}(+$ jets $/ \gamma)$ & VJets \\
W- $p_{\mathrm{T}}$ N-jet dep. & $\mathrm{W}(+$ jets $/ \gamma)$ & VJets \\
Instantaneous luminosity & All & VJets \\
Beamspot $z$-position & All & VJets \\
WW $p_{T}$ & Diboson WW & MC@NLO \\
WW $\Delta \phi$ & Diboson WW & SHERPA \\
H $p_{T}$ & gg $\rightarrow$ H $\rightarrow$ WW & HQT \\
Detector $\eta$ & All, inclusive & From data \\
Unclustered energy & All, N-jet sep. & From data \\
Jet1 $\eta$ & $\mathrm{Z} / \gamma^{*}, 1$-jet and 2 -jet & From data \\
Jet2 $\eta$ & $\mathrm{Z} / \gamma^{*}, 2$-jet & From data \\
$\Delta \mathrm{R}\left(j_{1}, j_{2}\right)$ & $\mathrm{Z} / \gamma^{*}, 2$-jet & From data \\
\hline
\end{tabular}


[1] M. K. Gaillard, P. D. Grannis, and F. J. Sciulli, Rev.Mod.Phys. 71, S96-S111 (1999), arXiv:hep-ph/9812285 [hep-ph].

[2] Particle Data Group Collaboration, K. Nakamura et al., J.Phys.G G37, 075021 (2010).

[3] P. W. Higgs, Phys. Lett. 12, 132-133 (1964).

[4] F. Englert and R. Brout, Phys. Rev. Lett. 13, 321-322 (1964).

[5] G. S. Guralnik, C. R. Hagen, and T. W. B. Kibble, Phys. Rev. Lett. 13, 585-587 (1964).

[6] The LEP Higgs Working Group Collaboration, R. Barate et al., Phys.Lett. B565, 61-75 (2003), arXiv:hep-ex/0306033 [hep-ex].

[7] LEP and SLD Collaboration, J. Alcaraz et al., arXiv:0911.2604 [hep-ex].

[8] T. Han and R. J. Zhang, Phys.Rev.Lett. 82, 25-28 (1999), arXiv:hep-ph/9807424 [hep-ph].

[9] CDF and DØ Collaboration, T. Aaltonen et al., Phys.Rev.Lett. 104, 061802 (2010), arXiv:1001.4162 [hep-ex].

[10] DØ Collaboration, "Combined upper limits on standard model higgs boson production in the WW, $\tau \tau$ and $\gamma \gamma$ decays modes in $8.2 \mathrm{fb}^{-1}$ of data from the $\mathrm{D} \varnothing$ experiment." DØ note 6183, 2011.

[11] G. Salam, "Hunting for Terascale physics at the LHC." Physics Colloquium, Princeton, Dec., 2010.

[12] G. F. Giudice, A zeptospace odyssey: a journey into the physics of the LHC. Oxford University Press, 2010.

[13] S. L. Glashow, Nucl. Phys. 22, 579-588 (1961). 
[14] S. Weinberg, Phys.Rev.Lett. 19, 1264-1266 (1967).

[15] A. Salam. Originally printed in "Svartholm: Elementary Particle Theory, Proceedings Of The Nobel Symposium Held 1968 At Lerum, Sweden," Stockholm 1968, 367-377.

[16] C. Quigg, Contemp.Phys. 48, 1-11 (2007), arXiv:0704.2045 [hep-ph].

[17] M. M. Kado and C. G. Tully, Ann.Rev.Nucl.Part.Sci. 52, 65-113 (2002).

[18] U. Aglietti, A. Belyaev, S. Berge, A. Blum, R. Bonciani, et al., arXiv:hep-ph/0612172 [hep-ph].

[19] N. Becerici Schmidt, S. A. Cetin, S. Istin, and S. Sultansoy, Eur. Phys. J. C66, 119126 (2010), arXiv:0908.2653 [hep-ph].

[20] A. Djouadi, J. Kalinowski, and M. Spira, Comput.Phys.Commun. 108, 56-74 (1998), arXiv:hep-ph/9704448 [hep-ph].

[21] L. M. Lederman, Sci. Am. 264, 26-33 (1991).

[22] H. J. Frisch, "Introduction to High Pt Physics at the Tevatron," in NATO Summer School Proceedings, Cargese. 1998.

[23] M. D. Shapiro and J. L. Siegrist, Annual Review of Nuclear and Particle Science 41, 97-132 (1991).

[24] T. Han, arXiv:hep-ph/0508097 [hep-ph].

[25] S. Abachi et al., Nucl. Instr. and Meth. A 338, 185 - 253 (1994).

[26] V. M. Abazov et al., Nucl. Instr. and Meth. A 565, 463 - 537 (2006).

[27] G. Landsberg, "Materials in the DØ upgrade." DØ note 2709, 1995.

[28] DØ Collaboration, S. N. Ahmed et al., Nucl. Instr. and Meth. A 634, 8 - 46 (2011).

[29] R. Angstadt et al., Nucl. Instr. and Meth. A 622, 298-310 (2010), arXiv:0911.2522 [physics.ins-det].

[30] A. Khanov, "HTF: histogramming method for finding tracks. The algorithm description." DØ note 3778, 2000.

[31] G. Borissov, "AA track finding algorithm." Presentation given at the DØ plenary meeting, 2003. http://www-d0.fnal.gov/atwork/adm/d0_private/2003-02-28/ adm_talk.ps.

[32] T. LeCompte and H. T. Diehl, Annu. Rev. Nucl. Part. Sci. 50, 71-117 (2000).

[33] J. Zhu, "Determination of electron energy scale and energy resolution using p14 Z $\rightarrow$ ee data." DØ note 4323, 2004. 
[34] M. Voutilainen and C. Royon, "Jet $p_{\mathrm{T}}$ resolution using v7.1 JES for p17 data." DØ note 5381, 2007.

[35] G. C. Blazey et al., arXiv:hep-ex/0005012.

[36] DØ Collaboration, V. M. Abazov et al., Nucl. Instr. and Meth. A 620, 490 - 517 (2010).

[37] M. Arthaud, F. Deliot, B. Tuchming, and V. Sharyy, "Muon momemtum oversmearing for p17 data." DØ note 5444, 2007.

[38] P. Calfayan, T. Gadfort, G. Hesketh, V. Lesne, M. Owen, R. Stroehmer, V. Sharyy, and B. Tuchming, "Muon identification certification for p17 data." DØ note 5157, 2006.

[39] O. Brandt, S. Cho, M. Cooke, M. Eads, D. Hedin, A. Santos, B. Tuchming, Y. Yatsunenko, and S. Youn, "Muon identication certification for the summer 2009 extended dataset." DØ note 6025, 2010.

[40] M. L. Mangano and T. J. Stelzer, Ann.Rev.Nucl.Part.Sci. 55, 555-588 (2005).

[41] S. Hoeche, F. Krauss, N. Lavesson, L. Lonnblad, M. Mangano, A. Schalicke, and S. Schumann, arXiv:hep-ph/0602031 [hep-ph].

[42] D. Gillberg, "Heavy Flavour Removal and Determination of Weighting Factors for AlPGEN W+jet Monte Carlo." DØ note 5129, 2006.

[43] R. Brun, F. Bruyant, M. Maire, A. C. McPherson, and P. Zanarini. CERN-DD-EE-841.

[44] G. Altarelli, "A QCD primer," in European School of High Energy Physics, N. Ellis and J. March-Russel, eds., vol. 631 of AIP Conference Proceedings, pp. 70-111, CERN. 2002. arXiv:hep-ph/0204179 [hep-ph].

[45] J. Elmsheuser, Search for the Higgs Boson in $H \rightarrow W W$ Decays at the DØ Experiment and Precise Muon Tracking. PhD thesis, Ludwig-Maximilians-Universität, 2004.

[46] P. Calfayan, "ORing single muon triggers in p17 data." DØ note 5329, 2008.

[47] B. Tuchming. Private communication, Feb., 2011.

[48] DØ Collaboration, Data Quality Group. Website. http://www-d0.fnal.gov/ computing/data_quality.

[49] T. Andeen et al., FERMILAB-TM-2365.

[50] B. Casey, M. Corcoran, Y. Enari, R. Partridge, M. Prewitt, and G. Snow, "The D $\varnothing$ RunIIb luminosity constant." DØ note 5945, 2009.

[51] T. Sjöstrand, P. Edén, C. Friberg, L. Lönnblad, G. Miu, S Mrenna, and E. Norrbin, Comput.Phys.Commun. 135, 238-259 (2001), arXiv:hep-ph/0010017 [hep-ph]. 
[52] J. Pumplin, D. R. Stump, J. Huston, H. L. Laia, P. M. Nadolsky, and W. K. Tung, JHEP 07, 012 (2002), arXiv: hep-ph/0201195.

[53] S. Catani, D. de Florian, M. Grazzini, and P. Nason, JHEP 07, 028 (2003), arXiv:hep-ph/0306211.

[54] C. Anastasiou, R. Boughezal, and F. Petriello, JHEP 0904, 003 (2009), arXiv:0811.3458 [hep-ph].

[55] D. de Florian and M. Grazzini, Phys.Lett. B674, 291-294 (2009), arXiv:0901. 2427 [hep-ph].

[56] Higgs Working Group Collaboration, K. A. Assamagan et al., arXiv:hep-ph/0406152 [hep-ph].

[57] O. Brein, A. Djouadi, and R. Harlander, Phys.Lett. B579, 149-156 (2004), arXiv:hep-ph/0307206 [hep-ph].

[58] M. L. Ciccolini, S. Dittmaier, and M. Kramer, Phys.Rev. D68, 073003 (2003), arXiv:hep-ph/0306234 [hep-ph].

[59] E. L. Berger and J. M. Campbell, Phys.Rev. D70, 073011 (2004), arXiv:hep-ph/0403194 [hep-ph].

[60] G. Bozzi, S. Catani, D. de Florian, and M. Grazzini, Nucl.Phys. B737, 73-120 (2006), arXiv:hep-ph/0508068 [hep-ph].

[61] M. L. Mangano, M. Moretti, F. Piccinini, R. Pittau, and A. D. Polosa, JHEP 0307, 001 (2003), arXiv:hep-ph/0206293 [hep-ph].

[62] R. Hamberg, W. L. van Neerven, and T. Matsuura, Nucl.Phys. B359, 343-405 (1991).

[63] A. D. Martin, R. G. Roberts, W. J. Stirling, and R.S. Thorne, Phys.Lett. B604, 6168 (2004), arXiv:hep-ph/0410230 [hep-ph].

[64] S. Moch and P. Uwer, Phys.Rev. D78, 034003 (2008), arXiv:0804.1476 [hep-ph].

[65] J. M. Campbell and R. K. Ellis, Phys.Rev. D60, 113006 (1999), arXiv:hep-ph/9905386 [hep-ph].

[66] M. Cooke, A. Croc, F. Déliot, H. Greenlee, A. S. Santos, V. Sharyy, B. Tuchming, M. Vesterinen, and T. R. Wyatt, "Muon momentum oversmearing update for p20 data." DØ note 6031, 2010.

[67] B. Casey, G. Facini, and M. Rangel, "Jet ID/VC Efficiencies and Scale Factors using Dijet events." DØ note 6058, 2010.

[68] I. M. Anghel, C. E. Gerber, E. Shabalina, and T. Ten, "Studies of taggability versus number of primary vertices for p17 data." DØ note 5240, 2006. 
[69] S. Calvet, "Taggability scale factors for the dijet topology for summer conferences 2009." DØ note 5944, 2009.

[70] M. Shamim and T. Bolton, "Generator Level Reweighting of pT of Z Boson." D note 5565, 2008.

[71] D. Boline, "Jet Multiplicity Dependent Reweighting of the Z Boson pT in p20." DØ note 5569, 2008.

[72] G. Hesketh, "W $p_{\mathrm{T}}$ re-weighting for AlPGEN and PythiA ." DØ note 5786, 2008.

[73] DØ Collaboration, V. M. Abazov et al., Phys.Rev.Lett. 102, 161801 (2009), arXiv:0810.3873 [hep-ex].

[74] S. Frixione and B. R. Webber, JHEP 0206, 029 (2002), arXiv: hep-ph/0204244 [hep-ph].

[75] DØ Collaboration, V. M. Abazov et al., Phys. Rev. Lett. 105, 251801 (2010), arXiv:1008.3564 [hep-ex].

[76] A. Schwartzman, "Missing $E_{\mathrm{T}}$ significance algorithm in RunII data." DØ note 4254, 2003.

[77] G. Arnison et al., Physics Letters B 122, 103-116 (1983).

[78] C. G. Lester and D. J. Summers, Phys.Lett. B463, 99-103 (1999), arXiv:hep-ph/9906349 [hep-ph].

[79] H. C. Cheng and Z. Han, JHEP 0812, 063 (2008), arXiv:0810.5178 [hep-ph].

[80] A. Hocker, J. Stelzer, F. Tegenfeldt, H. Voss, A. Voss, K. Christov, S. Henrot-Versille, M. Jachowski, A. Krasznahorkay, Y. Mahalalel, X. Prudent, and P. Speckmayer, PoS ACAT, 040 (2007), arXiv:physics/0703039 [PHYSICS].

[81] R. Brun and F. Rademakers, Nucl.Instrum.Meth. A389, 81 - 86 (1997). http:// root.cern.ch.

[82] DØ Collaboration, W. Fisher. FERMILAB-TM-2386-E.

[83] A. L. Read, "Modified frequentist analysis of search results (the $\mathrm{CL}_{s}$ method)," in Workshop On Confidence Limits, Proceedings, p. 21. 2000. CERN-OPEN-2000-205.

[84] T. Junk, Nucl.Instrum.Meth. A434, 435-443 (1999), arXiv:hep-ex/9902006 [hep-ex].

[85] The CDF Collaboration, T. Aaltonen et al., Phys. Rev. Lett. 104, 061803 (2010), arXiv:1001.4468 [hep-ex].

[86] G. D. Kribs, T. Plehn, M. Spannowsky, and T. M. P. Tait, Phys. Rev. D76, 075016 (2007), arXiv:0706.3718 [hep-ph]. 
[87] CDF and DØ Collaboration, T. Aaltonen et al., Phys. Rev. D 82, 011102 (2010), arXiv:1005.3216 [hep-ex].

[88] The DØ Collaboration, V. M. Abazov et al., Phys.Rev.Lett. 104, 061804 (2010), arXiv:1001.4481 [hep-ex].

[89] CDF and DØ Collaboration, T. Aaltonen et al., arXiv:1103.3233 [hep-ex]. 